\title{
Quadruply-graded colored homology of knots
}

\author{
by \\ Eugene Gorsky (Davis, CA, and Moscow), \\ Sergei Gukov (Pasadena, CA, and Bonn) and \\ Marko Stošić (Lisbon and Belgrade)
}

\begin{abstract}
We conjecture the existence of four independent gradings in colored HOMFLYPT homology, and make qualitative predictions of various interesting structures and symmetries in the colored homology of arbitrary knots. We propose an explicit conjectural description for the rectangular colored homology of torus knots, and identify the new gradings in this context. While some of these structures have a natural interpretation in the physical realization of knot homologies based on counting supersymmetric configurations (BPS states, instantons, and vortices), others are completely new. They suggest new geometric and physical realizations of colored HOMFLYPT homology as the Hochschild homology of the category of branes in a Landau-Ginzburg B-model or, equivalently, in the mirror A-model. Supergroups and supermanifolds are surprisingly ubiquitous in all aspects of this work.
\end{abstract}

\section{Contents}

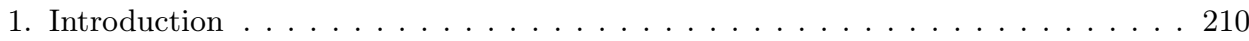

$1.1 . \quad$ Fourth grading . . . . . . . . . . . . . . . . . . . . . . 213

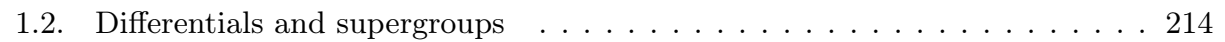

1.3. Main conjectures . . . . . . . . . . . . . . . . . . . . . . 214

1.4. Structure of the paper . . . . . . . . . . . . . . . . . . . . . 215

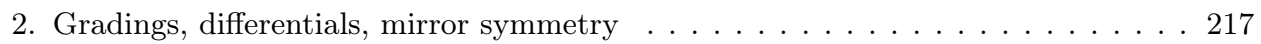

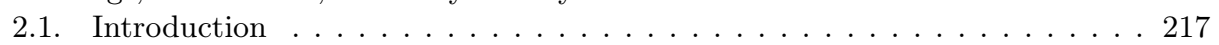

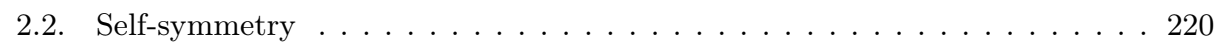

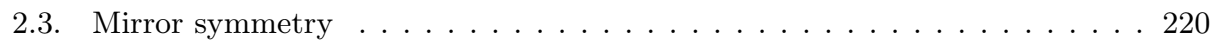

2.4. $\operatorname{gl}(n \mid m)$ differentials . . . . . . . . . . . . . . . . . . . . . . . 221

2.5. Colored differentials . . . . . . . . . . . . . . . . . . . . . 223

2.6. Representation theory interpretation of colored differentials . . . . . . . 227

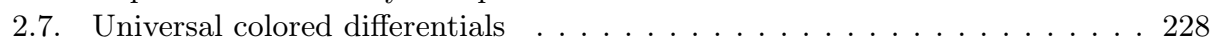

2010 Mathematics Subject Classification: Primary 57M27; Secondary 81T30, 20C08.

Key words and phrases: knot homology, colored HOMFLYPT invariants, BPS invariants, differentials, Lie superalgebras.

Received 28 October 2014; revised 21 February 2017.

Published online 3 September 2018. 
2.8. Refined exponential growth . . . . . . . . . . . . . . . . . . . . . . . 229

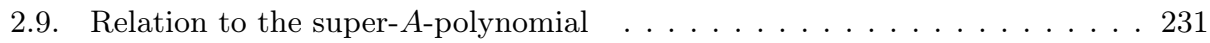

2.10. Symmetric and antisymmetric representations . . . . . . . . . . . . . 232

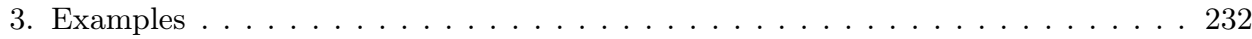

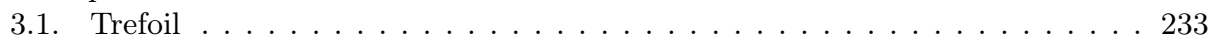

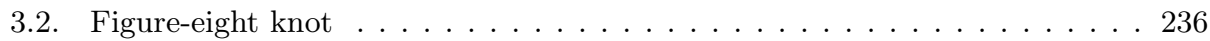

3.3. (3,4)-torus knot . . . . . . . . . . . . . . . . . . . . . . . 237

3.4. $\quad(2,2)$-representation . . . . . . . . . . . . . . . . . . . . . . . . . . . . 239

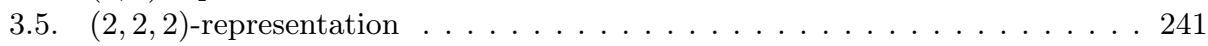

4. Bosonic and fermionic generators . . . . . . . . . . . . . . . . . 243

4.1. The algebra of BPS states and knot cobordisms . . . . . . . . . . . 243

4.1.1. Supersymmetry and $\mathrm{gl}(n \mid m)$ representations . . . . . . . . . . . . 247

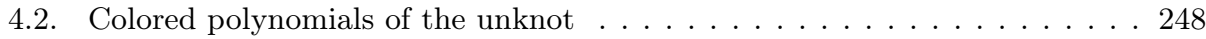

4.3. Schur-Weyl duality . . . . . . . . . . . . . . . . . . . . . . . 250

4.4. Colored homology of the unknot . . . . . . . . . . . . . . . . . . . 251

4.5. Potentials and differentials: antisymmetric case . . . . . . . . . . . . 252

4.6. Potentials and differentials: extension to $n \lambda \ldots \ldots \ldots$. . . . . . . . 254

4.7. Hilbert scheme interpretation . . . . . . . . . . . . . . . . . . 257

5. Colored HOMFLYPT homology for torus knots . . . . . . . . . . . . . . . 260

5.1. Colored HOMFLYPT polynomials of torus knots . . . . . . . . . . . . 260

5.2. Stable HOMFLYPT homology of torus knots . . . . . . . . . . . . . . . . 261

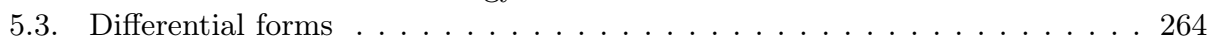

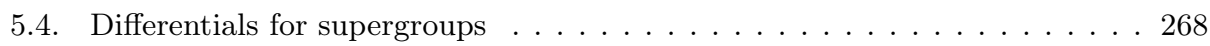

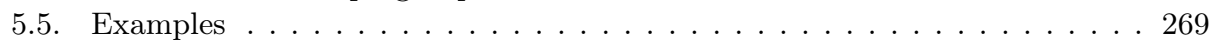

6. HOMFLYPT homology from A-model and B-model . . . . . . . . . . . . . . . 271

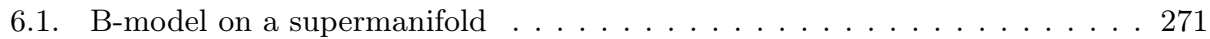

6.2. B-branes and open B-model . . . . . . . . . . . . . . . . . . 275

6.3. HOMFLYPT homology from symplectic geometry . . . . . . . . . . . . . . . 277

6.4. "Bottom row" of the colored HOMFLYPT homology . . . . . . . . . . . . . 278

Appendix A. Rectangle-colored invariants of $(2, m)$-torus knots . . . . . . . . . . . 284

Appendix B. A proposal for the $(2,1)$-colored trefoil . . . . . . . . . . . . . . . . 285

Appendix C. The unreduced colored HOMFLYPT homology . . . . . . . . . . . . . 287

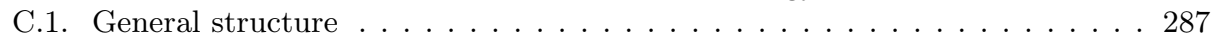

C.2. The unreduced $\mathrm{sl}(N)$-colored HOMFLYPT homology . . . . . . . . . . . . 288

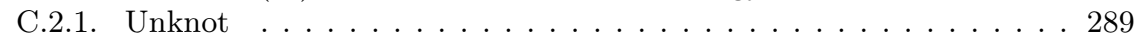

C.2.2. Trefoil . . . . . . . . . . . . . . . . . . . . 290

C.2.3. Figure-eight knot . . . . . . . . . . . . . . . . . . . . . . . 291

C.3. Comparison with the algebraic model . . . . . . . . . . . . . . 293

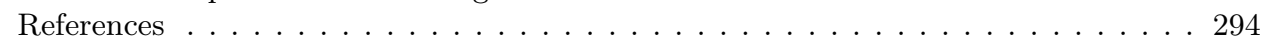

1. Introduction. The categorification of quantum knot invariants started with Khovanov's seminal paper [56], where he defined a doubly-graded homology theory whose homotopy type is an invariant of a knot, and such that its graded Euler characteristic is equal to the Jones polynomial. This opened a fast-growing field in low-dimensional topology and started work on categorification of other quantum knot invariants. Khovanov, Rozansky, Cautis, Kamnitzer and others [57, 58, 12, 13, 11] categorified all $\operatorname{sl}(N)$ Reshetikhin-Turaev invariants colored by arbitrary representations. 
We will denote by $P^{\mathfrak{g}, \lambda}(K)(q)$ the Reshetikhin-Turaev invariants of a knot $K$ colored by a representation $\lambda$ of a Lie algebra $\mathfrak{g}$. For a fixed Young diagram $\lambda$ and a knot $K$, the $\operatorname{sl}(N)$ quantum polynomials $P^{\mathrm{sl}(N), \lambda}(K)(q)$ can be organized in a single two-variable $\lambda$-colored HOMFLYPT polynomial $P^{\lambda}(K)(a, q)$ :

$$
P^{\mathrm{sl}(N), \lambda}(K)(q)=P^{\lambda}(K)\left(a=q^{N}, q\right) .
$$

Throughout the paper, we are mainly focusing on the reduced polynomials, i.e. normalized so that the value of the unknot is equal to 1 , and unless otherwise stated, the (colored) HOMFLYPT polynomial means the reduced (colored) HOMFLYPT polynomial $\left({ }^{1}\right)$.

A remarkable property of the quantum knot invariants and their categorifications is that they are related to many different areas of mathematics and physics, all of them bringing new viewpoints to this topic. The relationship between quantum field theory, in particular Chern-Simons theory, and the quantum knot invariants was discovered in the celebrated paper by Witten [94], and the physics insights have been extremely fruitful ever since. In the case of the categorifications, the realization of knot homologies as the space of certain BPS states gave rise to various predictions on the structure of (colored) HOMFLYPT homologies; see e.g. [42 for a friendly introduction and a review. Thus, Dunfield, the second author and Rasmussen [20] predicted the existence of a triply-graded knot homology theory which was later constructed by Khovanov and Rozansky:

THEOREM 1.1 ([58, 78]). There exists a triply-graded homology theory $\mathcal{H}^{\square}(K)$ whose Euler characteristic is given by the HOMFLYPT polynomial of $K$. For each $N>0$ there exists a spectral sequence which starts at $\mathcal{H}^{\square}(K)$ and converges to the $\mathrm{sl}(N)$ homology $\mathcal{H}^{\mathrm{sl}(N), \square}(K)$.

It was shown in [78] that this spectral sequence is induced by a differential $d_{N}$ on the chain complex corresponding to $\mathcal{H}^{\square}(K)$. By this reason, and motivated by the terminology of [20], from now on we will refer to the first differential in this spectral sequence as to $d_{N}$ or " $\operatorname{sl}(N)$ differential", while implicitly assuming the possible existence of higher differentials.

Conjecture $1.2([20])$. There is an involution $\phi$ on $\mathcal{H}^{\mathrm{sl}(N), \square}(K)$ extending the symmetry of the HOMFLYPT polynomial:

$$
P^{\square}(K)(a, q)=P^{\square}(K)\left(a, q^{-1}\right) .
$$

$\left({ }^{1}\right)$ Notation conventions: The reduced versions of the colored HOMFLYPT polynomial, colored HOMFLYPT homology, and the corresponding Poincaré polynomial are denoted by $P^{\lambda}(K), \mathcal{H}^{\lambda}(K)$, and $\mathcal{P}^{\lambda}(K)$, respectively. The unreduced versions are denoted by $\bar{P}^{\lambda}(K), \overline{\mathcal{H}}^{\lambda}(K)$, and $\overline{\mathcal{P}}^{\lambda}(K)$, respectively. 
Furthermore, there are negative differentials $d_{N}, N<0$, such that $\phi d_{N}=$ $d_{-N} \phi$, and an additional differential $d_{0}$ such that $\phi d_{0}=d_{0} \phi$.

A rigid structure predicted by Theorem 1.1 and Conjecture 1.2 enabled the explicit conjectural description of triply-graded homology of various knots [20, 35, 39, 40]. Although these conjectures mostly remain open, in some cases they are confirmed by the recent computations of Hogancamp et al. [50, 22].

In [44] the second and the third authors extended this picture and conjectured various structures in the colored HOMFLYPT homology in the case of the symmetric $\left(S^{r}\right)$ and antisymmetric $\left(\Lambda^{r}\right)$ representations. With such a representation $\lambda$ and a knot $K$ one can associate an $(a, q, t)$-graded vector space $\mathcal{H}^{\lambda}(K)$ such that its Euler characteristic with respect to the $t$-grading equals the $\lambda$-colored HOMFLYPT polynomial $P^{\lambda}(K)(a, q)$ of $K$. As in the uncolored case, these homology theories are expected to come with the collection of differentials corresponding to the $\operatorname{sl}(N)$ specializations. The main new feature is the existence of another collection of the so-called colored differentials which give "dynamics" in the sequence of the homology theories $\mathcal{H}^{S^{r}}(K)$, for various $r$. For every pair of nonnegative integers $r$ and $k$ with $r>k$, 44] conjectured the existence of two different differentials $d_{S^{r} \rightarrow S^{k}}^{+}$ and $d_{S^{r} \rightarrow S^{k}}^{-}$on $\mathcal{H}^{S^{r}}(K)$ such that the homology of $\mathcal{H}^{S^{r}}(K)$ with respect to $d_{S^{r} \rightarrow S^{k}}^{ \pm}$is isomorphic to $\mathcal{H}^{S^{k}}(K)\left({ }^{2}\right)$.

The involution $\phi$ becomes the so-called mirror symmetry in colored homology: 44] conjectured an isomorphism between $\mathcal{H}^{S^{r}}(K)$ and $\mathcal{H}^{\Lambda^{r}}(K)$ preserving the $a$-grading and reversing the $q$-grading. Furthermore, 44] conjectured that the exponential growth property holds for certain classes of knots, i.e. the size of $S^{r}$-colored homology grows exponentially in $r$ :

$$
\operatorname{dim} \mathcal{H}^{S^{r}}(K)=\left(\operatorname{dim} \mathcal{H}^{\square}(K)\right)^{r} .
$$

Moreover, the refined exponential growth is valid for the two-variable $(q=1)$ specializations of the Poincaré polynomials of the corresponding homology theories. The consistency of all the conjectured properties was demonstrated in 44 by the explicit computation of homology groups for various knots and colors satisfying all of them (and conjecturally matching colored HOMFLYPT homology). Very recently, Wedrich partially proved the exponential growth conjecture:

THEOREM $1.3\left(\right.$ (93]). There is a spectral sequence starting at $\mathcal{H}^{\Lambda^{r}}(K)$ and converging to $\left(\operatorname{dim} \mathcal{H}^{\square}(K)\right)^{r}$ which preserves the homological grading.

$\left({ }^{2}\right)$ As above, these differentials are expected to be defined on the level of chain complexes and induce the spectral sequences in homology. 
As a corollary, 1.3 would follow from the collapse of this spectral sequence given the mirror symmetry between $S^{r}$ and $\Lambda^{r}$.

1.1. Fourth grading. In the present article we give a more quantitative description of the conjectures of [44] and extend them to rectangular diagrams $\lambda$. This allows us to give a more unified treatment of $\Lambda^{r}$ - and $S^{r}$-colored homology. The major novelty is endowing the space $\mathcal{H}^{\lambda}(K)$ with the fourth grading. We conjecture that the colored HOMFLYPT homology $\mathcal{H}^{\lambda}(K)$ of a knot $K$ carries four independent gradings: apart from the $a$ - and $q$-gradings from the polynomial invariants, there are two homological gradings, denoted $t_{r}$ and $t_{c}$. If $\mathcal{P}_{r}^{\lambda}(K)$ and $\mathcal{P}_{c}^{\lambda}(K)$ are three-variable Poincaré polynomials of $\mathcal{H}^{\lambda}(K)$ with respect to $t_{r^{-}}$and $t_{c^{-}}$-gradings, respectively, then

$$
\mathcal{P}_{r}^{\lambda}(K)\left(a, q, t_{r}=-1\right)=\mathcal{P}_{c}^{\lambda}(K)\left(a, q, t_{c}=-1\right)=P^{\lambda}(K)(a, q) .
$$

The appearance of the fourth grading is yet mysterious for us from the geometric point of view, but it seems to be inevitable. Let us list some evidence for this.

First of all, in such a way we managed to reconcile the two different conventions for the homological grading in the case of symmetric representations: $t_{r}$ is the $t$-grading assigned to a generator of $\mathcal{H}^{\lambda}(K)$ in the grading conventions of [44], whereas one can interpret $t_{c}$ as the $t$-grading assigned to a generator of $\mathcal{H}^{\lambda}(K)$ in the grading conventions of [2, 21, 20, 45]. The "mirror symmetry" exchanges the two grading conventions. The quadruply-graded theory $\mathcal{H}^{\lambda}(K)$ gives precise re-gradings of all colored isomorphisms, enables explicit expression in all gradings for the mirror symmetry, and makes the exponential growth property manifest as a fully refined exponential growth property of three-variable polynomials. In addition, we found a new selfsymmetry of $\mathcal{H}^{\lambda}(K)$. All of this becomes particularly elegant when expressed in terms of an auxiliary grading, called the $Q$-grading, defined in the following simple way when $\lambda$ is a rectangular Young diagram with $R$ rows:

$$
Q=\frac{q+t_{r}-t_{c}}{R}
$$

This new grading, which can be considered as a certain "corrected" $q$-grading, cannot be seen at the decategorified, polynomial level, and as the formula indicates, both $t$-gradings are needed for its definition. To indicate the regrading from $\left(a, q, t_{r}, t_{c}\right)$ to $\left(a, Q, t_{r}, t_{c}\right)$, we will use the notation $\widetilde{\mathcal{H}}^{\lambda}(K)$ and refer to it as the tilde-version of colored HOMFLYPT homology. It is given by

$$
\widetilde{\mathcal{H}}_{i, j, k, l}^{\lambda}(K):=\mathcal{H}_{i, R j-k+l, k, l}^{\lambda}(K) .
$$

We note that only in the uncolored case the $t_{r^{-}}$and $t_{c^{-}}$gradings coincide (as do the $Q$ - and $q$-gradings), and the resulting homology is triply-graded in agreement with [20]. 
1.2. Differentials and supergroups. The second major novelty is that we extend the differentials $d_{N}$ to a two-dimensional family of differentials $d_{n \mid m}$ labeled by Lie superalgebras $\operatorname{gl}(n \mid m)$. This gives a much more natural treatment of the differentials $d_{N}$ for nonpositive $N$, together with their gradings. We expect that these new differentials are nontrivial even in the uncolored homology (for sufficiently large knots) and bring an interesting structure both to colored and uncolored knot homology. We also explicitly describe the interaction between the differentials $d_{n \mid m}$ and the colored differentials for the rectangle-colored homology.

While it still deserves a much deeper understanding, the appearance of $\mathrm{gl}(n \mid m)$ is natural for a number of reasons. Primarily, the representation theory of $\operatorname{gl}(n \mid m)$ at least partially explains the behavior of the colored differentials. For each rectangular Young diagram $\lambda$ we define colored differentials removing any number of columns or rows from $\lambda$. These differentials naturally appear from the identification of the representations of the superalgebra $\operatorname{gl}(n \mid m)$ labeled by two different rectangles $\lambda$ and $\mu$. It turns out that these are such that $\mu$ is obtained from $\lambda$ by erasing some of its rows or some of its columns. Then the corresponding colored differential, $d_{\lambda \rightarrow \mu}$, closes the following commutative diagram:

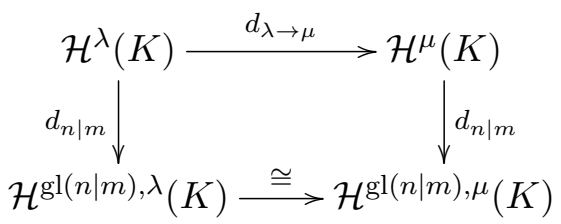

In addition, supergroups and the corresponding Lie superalgebras are familiar in the study of brane/antibrane systems [91, 95]: much like a collection of $N$ coincident $\mathrm{D}$-branes carries a gauge bundle with the structure group $U(N)$, a similar system of $n$ branes and $m$ antibranes carries $U(n \mid m)$ gauge symmetry. Therefore, since many physical realizations of knot homologies that we encounter in Sections 4.1, 2.4, 2.6, and 6.4 are based on branes, it is not inconceivable that the appearance of supergroups and superalgebras is rooted there (although we will not try to pursue this interpretation in the present paper).

After the first version of this paper was posted on arXiv, a lot of work on polynomial invariants and homologies for $\mathrm{gl}(n \mid m)$ was done in mathematics and physics. We refer the reader to [90, 75, 76, 66, 81] and references therein.

1.3. Main conjectures. All these structural properties are collected in the conjectures in Section 2. The homology theories $\mathcal{H}^{\lambda}$ categorifying the (reduced) HOMFLYPT polynomials $P^{\lambda}$ are quadruply $\left(a, q, t_{r}, t_{c}\right)$-graded. We focus on the rectangular $R \times C$ Young diagram $\lambda$, and the auxiliary $Q$-grading is given by 1.5 . 
The conjectures include the mirror symmetry between $\mathcal{H}^{\lambda}(K)$ and $\mathcal{H}^{\lambda^{t}}(K)$, that preserves the $a$-grading and reverses the $q$-grading. Furthermore, there is a new self-symmetry on $\mathcal{H}^{\lambda}(K)$ that reverses the $Q$-grading.

We also predict the existence of color-changing differentials: for every rectangular diagram $\mu$ that is obtained from $\lambda$ by erasing either some of its rows or some of its columns, there exist two different differentials, denoted $d_{\lambda \rightarrow \mu}^{+}$and $d_{\lambda \rightarrow \mu}^{-}$, on $\mathcal{H}^{\lambda}(K)$ such that the homology of $\mathcal{H}^{\lambda}(K)$ with respect to any of those two differentials is isomorphic to $\mathcal{H}^{\mu}(K)$. And finally, on $\mathcal{H}^{\lambda}(K)$ we predict the existence of a collection $\left\{d_{n \mid m}\right\}$ of mutually anticommuting differentials, labeled by pairs of nonnegative integers $(n, m)$, with $n \geq R$ or $m \geq C$, such that:

$$
H^{*}\left(\mathcal{H}^{\lambda}(K), d_{n \mid m}\right) \cong \mathcal{H}^{\operatorname{gl}(n \mid m), \lambda}(K) .
$$

Here, $\mathcal{H}^{\operatorname{gl}(n \mid m), \lambda}(K)$ stands for the $(\operatorname{gl}(n \mid m), \lambda)$ homology that should categorify the quantum polynomial invariant of $K$ that corresponds to the representation $\lambda$ of $\operatorname{gl}(n \mid m)$. Moreover, the colored differentials $d_{\lambda \rightarrow \mu}$ are related to the specialization differentials $d_{n \mid m}$ for suitable $n$ and $m$, so that they form the commutative diagram (1.7).

All four gradings are essential for making the isomorphisms and symmetries from above completely explicit. In addition, all these explicit expressions have a particularly nice and simple form, as we shall describe in detail in Section 2 .

Among many interesting additional structures, we emphasize the refined exponential growth in the case of the 2-bridge knots and torus knots:

Conjecture 1.4. Let $K$ be a 2-bridge knot or a torus knot. Then the dimension of the $r$-symmetric homology $\mathcal{H}^{S^{r}}(K)$ grows exponentially with $r$. More explicitly, the corresponding $\left(a, Q, t_{r}\right)$-graded Poincaré polynomials satisfy

$$
\mathcal{P}^{S^{r}}(K)\left(a, Q, t_{r}\right)=\left(\mathcal{P}^{\square}(K)\left(a, Q, t_{r}\right)\right)^{r} .
$$

1.4. Structure of the paper. In Section 2 we describe the general structures on colored HOMFLYPT homology, together with the gl $(n \mid m)$ and colored differentials. We describe explicitly the degrees of all colored differentials and re-gradings in the corresponding colored isomorphisms. We end the section with the two particular features in the case of the symmetric representations: the $\delta$-grading and the existence of the HFK-like differential on the $S^{r}$-colored homology.

Section 3 contains models of the quadruply-graded homologies for various knots and representations. Due to a large number of predicted properties, such computations present a highly nontrivial consistency check for the conjectures. 
In Section 4 we describe a model for the unreduced colored homology of the unknot. It is a free supercommutative algebra $\mathcal{A}$ with one even and one odd generator per each box of the diagram $\lambda$. For the representation $S^{n}$, this algebra can be naturally identified with the algebra of $S_{n}$-invariant differential forms on $\mathbb{C}^{n}$. Using the physics of BPS states, we describe some of these even and odd generators geometrically. We also check that the Poincaré polynomial for this algebra agrees with the evaluation formulas for the Macdonald polynomial, which various authors [2, 14, 21] recently assign to the unknot in the refined Chern-Simons theory.

Both $d_{m \mid n}$ and the colored differentials can be interpreted as the Koszul differentials on this algebra, sending odd generators to certain polynomials in even generators. We show that the approach of [45, where the homology of the unknot is interpreted as the Jacobi ring of a certain potential $W$, fits into this framework if the Koszul complex is associated with the partial derivatives of $W$ :

$$
d\left(\xi_{i}\right)=\frac{\partial W}{\partial x_{i}}, \quad d\left(x_{i}\right)=0 .
$$

Using this model, we also derive the equations for the colored differentials: if the representations of $\operatorname{gl}(m \mid n)$ labeled by $\lambda$ and $\mu$ give rise to isomorphic knot homologies, we show that in some examples the isomorphism between the corresponding Jacobi rings follows from the equation

$$
W_{m \mid n}^{\lambda}\left(x_{1}, \ldots, x_{|\lambda|}\right)=W_{m \mid n}^{\mu}\left(x_{1}, \ldots, x_{|\mu|}\right)+W_{\lambda \rightarrow \mu}\left(x_{|\mu|+1}, \ldots, x_{|\lambda|}\right),
$$

where $W_{\lambda \rightarrow \mu}$ is a nondegenerate quadratic function. We conjecture that the colored differential $d_{\lambda \rightarrow \mu}$ is a Koszul differential associated with the partial derivatives of $W_{\lambda \rightarrow \mu}$. We end in Section 4.7 with the Hilbert scheme interpretation of colored HOMFLYPT homology.

Section 5 is devoted to torus knots. In Section 5.2 we recall the statement about the stabilization of the HOMFLYPT polynomials of $(p, q)$-torus knots in the limit $q \rightarrow \infty$. We relate such a stable $\lambda$-colored invariant of a $(p, \infty)$ torus knot to the invariant of the $p \lambda$-colored unknot. Then, we conjecture a similar relation between the corresponding colored homology theories of stable torus knots and that of the unknot. The $\lambda$-colored homology of the finite $(p, q)$-torus knot is expected to be a certain quotient of this stable homology. This allows us to use the algebraic description of the homology of the unknot, and in Section 5.3 we give a precise description of the generators in the rectangular homology of a torus knot and their quadruple gradings. We describe and check the structural properties of colored homology using this algebraic model. We show the existence of a symmetry between $\lambda$ - and $\lambda^{t}$-colored homology, exchanging $t_{r^{-}}$and $t_{c^{-}}$-gradings and preserving the $Q$ grading. We check the refined exponential growth conjecture for the stable 
homology of torus knots. We also describe the self-duality involution and the action of differentials in the rectangular homology of torus knots.

Generalizing the results of [23] and conjectures of [40], we identify the colored homology of torus knots with the certain representations of the rational Cherednik algebra. For $\lambda=S^{r}$, we make this description explicit and describe the space $\mathcal{H}^{S^{r}}(T(p, q))$ as a space of differential forms on a certain nonreduced scheme

$$
\mathcal{M}_{p, q}(r):=\left\{\left(u_{r+1}, \ldots, u_{p r} ; v_{r+1}, \ldots, v_{q r}\right) \mid U(z)^{q}=V(z)^{p}\right\},
$$

where the polynomials $U(z)$ and $V(z)$ are defined by

$$
U(z)=1+z^{r+1} u_{r+1}+\cdots+z^{p r} u_{p r}, \quad V(z)=1+z^{r+1} v_{r+1}+\cdots+z^{q r} v_{q r} .
$$

This description is ideally suited for a realization of colored HOMFLYPT homology via a Landau-Ginzburg B-model, which is the subject of Section 6 . Using mirror symmetry we also reformulate it in terms of the A-model and Lagrangian Floer homology. We also provide some explicit formulas for the differentials acting on HOMFLYPT homology and describe $\mathcal{H}^{S^{r}}(T(p, q))$ as a Jacobi ring of a certain potential on a supermanifold.

Finally, Section 6.4 is devoted to the "bottom row" of HOMFLYPT homology, i.e. the $a=0$ limit. We compare it to certain combinatorial models of Haglund et al., generalizing the models for $q, t$-Catalan numbers which played an important role in [40, and to the geometric models for coupled instanton-vortex systems proposed in [19].

\section{Gradings, differentials, mirror symmetry}

2.1. Introduction. Let $\mathcal{H}^{\lambda}(K)$ denote the reduced $\lambda$-colored HOMFLYPT homology of a knot $K$. We focus on rectangular Young diagrams $\lambda$, i.e. Young diagrams which have the form of a rectangle with $R$ rows and $C$ columns, briefly an $R \times C$ rectangle. This is a large class of representations, which includes as particular cases all symmetric representations $(R=1)$ and antisymmetric ones $(C=1)$. Our main results for $\mathcal{H}^{\lambda}(K)$ can be split into two groups, which match together perfectly. First, we conjecture that certain grading-independent structures exist:

Conjecture 2.1. For every knot $K$, there exists a homology $\mathcal{H}^{\lambda}(K)$ with the following properties:

- $\mathcal{H}^{\lambda}(K)$ is finite-dimensional.

- There are two symmetries on $\mathcal{H}^{\lambda}(K)$ :

- mirror symmetry that switches between $\lambda$ and its transpose partition $\lambda^{t}$ :

$$
\mathcal{H}^{\lambda}(K) \cong \mathcal{H}^{\lambda^{t}}(K)
$$

- self-symmetry on $\mathcal{H}^{\lambda}(K)$. 
- For every pair of nonnegative integers $(n, m)$ such that $n \geq R$ or $m \geq C\left[\left(^{3}\right)\right.$, there exists a collection of differentials $d_{n \mid m}$ on $\mathcal{H}^{\lambda}(K)$ that pairwise anticommute, and are such that the homology of $\mathcal{H}^{\lambda}(K)$ with respect to $d_{n \mid m}$ is isomorphic to the homology $\mathcal{H}^{\mathrm{gl}(n \mid m), \lambda}(K)$ that categorifies the quantum invariant of a knot $K$ labeled by a representation $\lambda$ of $\operatorname{gl}(n \mid m)$ :

$$
H^{*}\left(\mathcal{H}^{\lambda}(K), d_{n \mid m}\right)=\mathcal{H}^{\mathrm{gl}(n \mid m), \lambda}(K) .
$$

- For certain rectangular Young diagrams $\mu$ with $\mu \subset \lambda$, there exist colored differentials $d_{\lambda \rightarrow \mu}$ such that

$$
H^{*}\left(\mathcal{H}^{\lambda}(K), d_{\lambda \rightarrow \mu}\right)=\mathcal{H}^{\mu}(K) .
$$

- For large classes of knots, including torus knots and two-bridge knots, the size of the homology $\mathcal{H}^{\lambda}(K)$ is equal to the $|\lambda|$ th power of the dimension of $\mathcal{H}^{\square}(K)$. For an arbitrary knot $K$, such relation holds asymptotically as $|\lambda|$ tends to infinity.

A couple of remarks are in order regarding numerous structures on the colored HOMFLYPT homology predicted by Conjecture 2.1.

REMARK 2.2. While mirror symmetry that switches between $\lambda$ and its transpose partition $\lambda^{t}$ categorifies the known relation for the colored HOMFLYPT polynomials,

$$
P^{\lambda}(K)(a, q)=P^{\lambda^{t}}(K)\left(a, q^{-1}\right),
$$

self-symmetry is a completely new symmetry, which does not categorify any polynomial relation. It uses homological grading in a nontrivial way, and exists only at the homological level.

REMARK 2.3. Another novelty of this paper is the introduction of the two-parameter collection of differentials $d_{n \mid m}$ and the relevance of the superalgebras $\operatorname{gl}(n \mid m)\left[{ }^{4}\right)$. The main group of colored differentials, namely the group of $2(R+C-1)$ differentials, is directly related to the differentials $d_{n \mid m}$. More precisely, for every Young diagram $\mu$ that is obtained from $\lambda$ by removing either some of its rows or some of its columns, there should be two different differentials of the form $d_{\lambda \rightarrow \mu}$ such that

$$
H^{*}\left(\mathcal{H}^{\lambda}(K), d_{\lambda \rightarrow \mu}\right)=\mathcal{H}^{\mu}(K) .
$$

They "lift" the relation $\mathcal{H}^{\mathrm{gl}(n \mid m), \lambda}(K) \cong \mathcal{H}^{\mathrm{gl}(n \mid m), \mu}(K)$ coming from the particular relationship between $\lambda$ and $\mu$ as $\operatorname{gl}(n \mid m)$-representations for appropriate values of $n$ and $m$, and we also denote that differential by $d_{n \mid m}^{\text {col }}$. In

$\left({ }^{3}\right)$ For other values of $m$ and $n$ the differentials may exist in the unreduced theory, which we do not cover in detail here.

$\left({ }^{4}\right)$ Although some of these operators were implicitly discussed in 35] for uncolored homology, their relation to supergroups was not understood there. 
other words, we have the following commutative diagram:

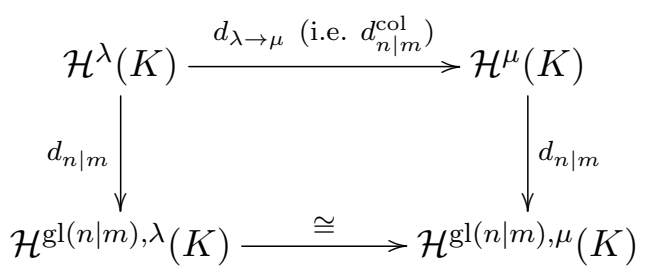

Here the arrow going from $\mathcal{H}_{1}$ to $\mathcal{H}_{2}$ labeled by $d$ means that the differential $d$ on $\mathcal{H}_{1}$ is such that $H^{*}\left(\mathcal{H}_{1}, d\right) \cong \mathcal{H}_{2}$. Note that the two differentials denoted $d_{n \mid m}$ are essentially different since they are defined on different homology theories. On $\mathcal{H}^{\lambda}(K)$ the differentials $d_{n \mid m}$ and $d_{n \mid m}^{\text {col }}$ share many properties. In particular, frequently the differential $d_{n \mid m}$ on $\mathcal{H}^{\mu}(K)$ acts trivially, and then on $\mathcal{H}^{\lambda}(K) d_{n \mid m}$ and $d_{n \mid m}^{\text {col }}$ coincide.

Our second major conjecture is that the colored HOMFLYPT homology $\mathcal{H}^{\lambda}(K)$ of a knot $K$ is quadruply-graded. We denote these four gradings by $a, q, t_{r}, t_{c}$. Thus, apart from the $a$ - and $q$-gradings associated to each generator, we also have two homological $t$-gradings. Any of the two $t$-gradings gives a categorification of $P^{\lambda}(K)$, i.e. forgetting one of them gives a triply-graded categorification of the colored HOMFLYPT polynomial. In other words, if we denote the (four-variable) Poincaré polynomial of $\mathcal{H}^{\lambda}(K)$ by $\mathcal{P}^{\lambda}(K)\left(a, q, t_{r}, t_{c}\right)$, then we have

$$
\begin{aligned}
\mathcal{P}^{\lambda}(K)\left(a, q, t_{r}=-1, t_{c}=1\right) & =\mathcal{P}^{\lambda}(K)\left(a, q, t_{r}=1, t_{c}=-1\right) \\
& =P^{\lambda}(K)(a, q) .
\end{aligned}
$$

We also introduce another auxiliary grading, called the $Q$-grading, defined by

$$
Q=\frac{q+t_{r}-t_{c}}{R}
$$

This new grading, which can be considered as a "corrected" q-grading, cannot be seen on the decategorified, polynomial level, and as the formula indicates, both $t$-gradings are needed for its definition. Finally, the symmetries get the nicest form when the homology is written in $\left(a, Q, t_{r}, t_{c}\right)$-gradings. Because of a linear relation between the gradings (2.6), this is just a simple re-grading of $\mathcal{H}^{\lambda}(K)$. However, due to its importance, we give it a special name, $\widetilde{\mathcal{H}}^{\lambda}(K)$, and refer to it as the tilde-version of colored HOMFLYPT homology. Explicitly, it is given by

$$
\widetilde{\mathcal{H}}_{i, j, k, l}^{\lambda}(K):=\mathcal{H}_{i, R j-k+l, k, l}^{\lambda}(K) .
$$

The only case when the two $t$-gradings coincide is the uncolored case, $\lambda=\square$, and the resulting homology is triply-graded in agreement with [20]. In this 
case the $q$ - and $Q$-gradings also coincide, and mirror symmetry and selfsymmetry are in fact the same symmetry.

In the following subsections we list more precise and explicit conjectures on the properties of the homologies $\widetilde{\mathcal{H}}^{\lambda}$ and differentials.

\subsection{Self-symmetry}

ConjeCture 2.4. The homology $\widetilde{\mathcal{H}}^{\lambda}(K)$ enjoys the following self-symmetry:

$$
\widetilde{\mathcal{H}}_{i, j, k, l}^{\lambda}(K) \cong \widetilde{\mathcal{H}}_{i,-j, k-R j, l-C j}^{\lambda}(K) \text {. }
$$

More precisely, we conjecture the existence of an involution $\Phi_{\lambda}: \mathcal{H}^{\lambda}(K)$ $\rightarrow \mathcal{H}^{\lambda}(K)$ such that for every generator $x$ of $\mathcal{H}^{\lambda}(K)$,

$$
\left(a, Q, t_{r}, t_{c}\right)\left[\Phi_{\lambda}(x)\right]=\left(a(x),-Q(x), t_{r}(x)-R Q(x), t_{c}(x)-C Q(x)\right) .
$$

Also, by (2.6) one has $q\left(\Phi_{\lambda}(x)\right)=q(x)-(R+C) Q(x)$. For $\lambda=\square$, the involution $\Phi_{\lambda}$ agrees with the involution $\phi$ described in Conjecture 1.2 .

For some knots, including torus knots (see below), we also conjecture that this involution comes from some version of the hard Lefschetz isomorphism. Namely, there exists an operator $L_{\lambda}: \mathcal{H}^{\lambda}(K) \rightarrow \mathcal{H}^{\lambda}(K)$ such that $\left({ }^{5}\right)$

$$
\left(a, q, Q, t_{r}, t_{c}\right)\left[L_{\lambda}\right]=(0, R+C, 2, R, C), \quad \Phi_{\lambda}(x)=L_{\lambda}^{-Q(x)} .
$$

2.3. Mirror symmetry. Based on results for symmetric and antisymmetric representations, it was conjectured [44 that colored HOMFLYPT homology enjoys mirror symmetry:

ConjeCture 2.5 (444). For any knot, there exists an isomorphism (called mirror symmetry) between $\lambda$ - and $\lambda^{t}$-colored HOMFLYPT homologies preserving the a-grading and reversing the q-grading.

However, to obtain the behavior of the $t$-gradings under this symmetry, one needs to introduce the fourth grading. We are able to write an explicit formula for the $t$-degrees change in the case of rectangular $\lambda$. We start with a different conjectural symmetry between $\lambda$ - and $\lambda^{t}$-colored homology.

CONJECTURE 2.6. In $\left(a, Q, t_{r}, t_{c}\right)$-gradings one has

$$
\widetilde{\mathcal{H}}_{i, j, k, l}^{\lambda^{t}}(K) \cong \widetilde{\mathcal{H}}_{i, j, l, k}^{\lambda}(K)
$$

To obtain an explicit quadruply-graded version of the mirror symmetry from (2.5), we combine self-symmetry $(2.8)$ and mirror symmetry 2.10$)$ :

$\left({ }^{5}\right)$ Note that according to the "dictionary" 4.4 this operator is a bound state of $R+C$ D0-branes. 
Corollary 2.7. In $\left(a, Q, t_{r}, t_{c}\right)$-gradings one has

$$
\widetilde{\mathcal{H}}_{i, j, k, l}^{\lambda^{t}}(K) \cong \widetilde{\mathcal{H}}_{i,-j, l-C j, k-R j}^{\lambda}(K) \text {. }
$$

The last equation enables one to write the explicit change of gradings in the mirror symmetry. Denote by $M_{\lambda}: \mathcal{H}^{\lambda} \rightarrow \mathcal{H}^{\lambda^{t}}$ the mirror symmetry isomorphism. Consider the gradings of a generator $x$ in $\mathcal{H}^{\lambda}$ theory and the gradings of the generator $M_{\lambda}(x)$ of $\mathcal{H}^{\lambda^{t}}$, denoted by $\left(\widehat{a}, \widehat{q}, \widehat{Q}, \widehat{t}_{r}, \widehat{t}_{c}\right)$, where $\lambda$ is an $R \times C$ rectangular Young diagram and $\lambda^{t}$ is a $C \times R$ rectangular Young diagram. They are related by the following transformation:

$$
\begin{aligned}
& \widehat{a}=a, \quad \widehat{q}=-q, \quad \widehat{Q}=-Q, \\
& \widehat{t_{r}}=t_{c}-C Q=\left(1+\frac{C}{R}\right) t_{c}-\frac{C}{R} t_{r}-\frac{C}{R} q, \\
& \widehat{t_{c}}=t_{r}-R Q=t_{c}-q .
\end{aligned}
$$

Since mirror symmetry from Conjecture 2.5 inverts the $q$-grading, it "categorifies" the following relation between colored HOMFLYPT polynomials:

$$
P^{\lambda}(K)(a, q)=P^{\lambda^{t}}(K)\left(a, q^{-1}\right) .
$$

We refer the reader to [90] for a representation-theoretic explanation and a diagrammatic version of 2.12 .

2.4. $\operatorname{gl}(n \mid m)$ differentials. The homology $\mathcal{H}^{\lambda}(K)$ comes with a large structure of pairwise anticommuting differentials on it. We conjecture that there exists a differential for every pair of nonnegative integers $(n, m)$ such that $n \geq R$ or $m \geq C$. These differentials should be related to the homologies corresponding to Lie superalgebras $\operatorname{gl}(n \mid m)$ and representations labeled by Young diagram $\lambda$. The condition that $n \geq R$ or $m \geq C$ gives precisely those pairs $(n, m)$ for which the Lie superalgebra $\mathrm{gl}(n \mid m)$ has an irreducible representation labeled by a $\lambda$ (see Section 2.6). This generalizes the structure from [20] and [44, where the analogous differentials are labeled by a single integer $N$ and were related to the $\operatorname{sl}(N)$ representations, i.e. $\operatorname{sl}(N)$ specializations of the $\lambda$-colored HOMFLYPT polynomial when $N>0$. As we shall argue in this paper (see Section 4.1.1), the structure corresponding to Lie superalgebras is more natural, and the single parameter $N$ from [20] and [44] is just the superrank of the corresponding Lie superalgebra:

$$
N=n-m \text {. }
$$

CONJECTURE 2.8. For every pair $(n, m)$ of nonnegative integers such that $n \geq R$ or $m \geq C$ there exists a differential $d_{n \mid m}$ on the homology $\mathcal{H}^{\lambda}(K)$ satisfying the following properties: 
- Specialization:

$$
\left.\left(\mathcal{H}^{\lambda}(K), d_{n \mid m}\right)\right|_{a=q^{n-m}} \cong \mathcal{H}^{\mathrm{gl}(n \mid m), \lambda}(K) .
$$

- Anticommutativity: All $d_{n \mid m}$ pairwise anticommute.

The homology theories $\mathcal{H}^{\mathrm{gl}(n \mid m), \lambda}(K)$ for arbitrary $n$ and $m$ are still not rigorously defined. Nevertheless, they should categorify the $(n-m)$ specialization of the $\lambda$-colored HOMFLYPT polynomial. In other words, the Poincaré polynomial of $\mathcal{H}^{\mathrm{gl}(n \mid m), \lambda}(K)$ satisfies

$$
\mathcal{P}^{\mathrm{gl}(n \mid m), \lambda}(K)(q, t=-1)=P^{\lambda}(K)\left(a=q^{n-m}, q\right) .
$$

Although the right-hand side equals the quantum polynomial invariant associated with $\operatorname{gl}(n \mid m)$ representations labeled by $\lambda$, it only depends on the superrank of the Lie superalgebra [75, 76]. However, we expect that the homologies $\mathcal{H}^{\mathrm{gl}(n \mid m), \lambda}(K)$ for various pairs $(n, m)$ with fixed $n-m$ can in general be nonisomorphic. That is one of the reasons why we expect to have differentials parametrized by two integers instead of just one, as was predicted for the fundamental representation [20] and for the symmetric representations [44]. We will say more on this in Section 5.4

The main reason for the existence of such structures comes from the representation theory of the Lie superalgebras $\operatorname{gl}(n \mid m)$. The condition that $n \geq R$ or $m \geq C$ gives precisely those pairs $(n, m)$ for which the Lie superalgebra $\operatorname{gl}(n \mid m)$ has an irreducible representation labeled by $\lambda$. We denote by $\Omega$ the subset of the $(x, y)$-plane satisfying $x \geq 0, y \geq 0$ and $x \geq R$ or $y \geq C$. Then the set of all admissible pairs $(n, m)$ for which we have a differential $d_{n \mid m}$ consists exactly of the points from $\Omega$ whose both coordinates are integers.

The degrees of the differentials $d_{n \mid m}$ satisfy

$$
(a, q)\left[d_{n \mid m}\right]=(-2,2(n-m)),
$$

in agreement with the specialization $a=q^{n-m}$. The $t_{c}$-degree is equal to

$$
t_{c}\left[d_{n \mid m}\right]=-2 m-1 .
$$

The $t_{r}$-degree is more subtle, and is given in the next section; in particular, it depends on $R$ and $C$.

In the case of the fundamental [20] and the symmetric representations [44, the conjectured differentials $d_{N}$ were parametrized by a single integer $N$. It was argued that they correspond to $\operatorname{sl}(N)$ representations for $N>0$, while for other values of $N$ there was no clear representation-theoretic motivation. However, when observed from the $\operatorname{gl}(n \mid m)$ point of view, they become much more natural: $d_{N}$ becomes in fact precisely the differential $d_{n \mid m}$, where $n$ and $m$ are uniquely determined by requiring that $N=n-m$ and that $(n, m)$ lies on the boundary of $\Omega$ (see Figure 1 ). 


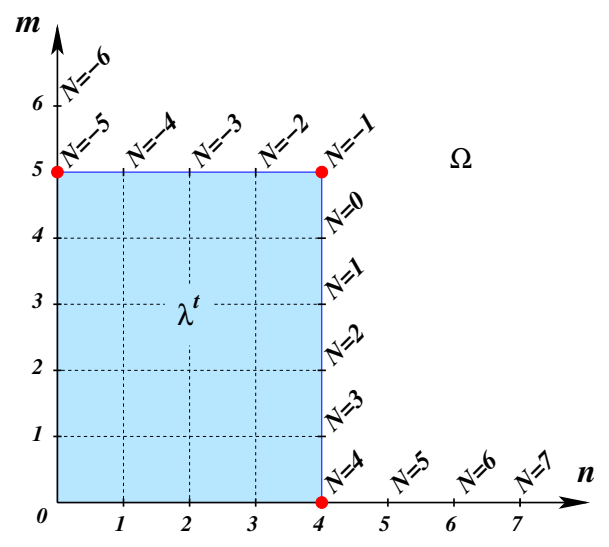

Fig. 1. The domain $\Omega$ can be visualized as the complement of $\lambda^{t}$ in the positive quadrant of the $(n, m)$-plane. Integer points on its boundary correspond to the differentials $d_{n \mid m}$, or equivalently $d_{N}$ with $N=n-m$, among which three are special. They correspond to the three corners of the domain $\Omega$ and are marked by bullets. The differentials for this example are shown in Figure 2

In the case of the fundamental representation, the $t$-degrees of the differentials $d_{N}$ were defined in a rather artificial way. By using the conventions above, we directly get the desired degrees. (Note that in the fundamental representations the two $t$-gradings coincide: $t_{r}=t_{c}=t$.) Indeed, for $N>0$ we have $d_{N}=d_{N \mid 0}$, and therefore according to (2.17) their $t$-degrees are equal to -1 . For $N=0$ we have $d_{0}=d_{1 \mid 1}$, so the $t$-degree of $d_{0}$ equals -3 , while for $N>0$ we have $d_{-N}=d_{0 \mid N}$, and so the $t$-degree of $d_{-N}$ equals $-2 N-1$, exactly as predicted in [20].

Although there are infinitely many integer pairs in $\Omega$, for a given knot $K$ only finitely many of the differentials $d_{n \mid m}$ can act nontrivially. Indeed, since $\mathcal{H}^{\lambda}(K)$ is finite-dimensional and since $q\left(d_{n \mid m}\right)=2(n-m)$ and $t_{c}\left(d_{n \mid m}\right)=$ $-2 m-1$, the differential $d_{n \mid m}$ becomes void on $\mathcal{H}^{\lambda}(K)$ for sufficiently large values of $n$ or $m$. Thus we have:

Conjecture 2.9. For a knot $K$ and a rectangular Young diagram $\lambda$,

$$
\mathcal{P}^{\mathrm{gl}(n \mid m), \lambda}(K)\left(q, t_{r}, t_{c}\right)=\mathcal{P}^{\lambda}(K)\left(a=q^{n-m}, q, t_{r}, t_{c}\right)
$$

when either $n$ or $m$ is sufficiently large.

2.5. Colored differentials. For every knot $K$, the homology theory $\mathcal{H}^{\lambda}(K)$ comes with a remarkable structure of the so-called colored differentials that allow passage to homology theories $\mathcal{H}^{\mu}(K)$ for various partitions $\mu \subset \lambda$ :

$$
H^{*}\left(\mathcal{H}^{\lambda}(K), d_{\lambda \rightarrow \mu}\right)=\mathcal{H}^{\mu}(K) .
$$


In particular, they are closely related to the differentials $d_{n \mid m}$ for suitable $n$ and $m$, as described in Remark 2.3 .

We conjecture that for each rectangular diagram $\mu$ that is obtained from $\lambda$ by removing either some rows or some columns, there exist two different differentials on $\mathcal{H}^{\lambda}$ realizing the isomorphism 2.19). As described in Remark 2.3. all these colored differentials are of the form $d_{n \mid m}^{\text {col }}$. However, in order to clearly indicate the color-changing property of the differentials, we also label them by a superscript " \pm " as in $d_{\lambda \rightarrow \mu}^{ \pm}$. One of them will be denoted as $d_{\lambda \rightarrow \mu}^{+}$: it has positive $Q$-degree (equal to +2 ), and will be referred to as the positive differential. The second differential has negative $Q$-degree (equal to -2 ) and will be denoted by $d_{\lambda \rightarrow \mu}^{-}$.

We will show that $d_{\lambda \rightarrow \mu}^{+}$and $d_{\lambda \rightarrow \mu}^{-}$are exchanged by the involution $\Phi_{\lambda}$. When $\mu=\emptyset$, the homology $\mathcal{H}^{\mu}$ is one-dimensional and the corresponding colored differentials are called canceling. All these differentials are deep generalizations of the differentials $d_{1}$ and $d_{-1}$ in the uncolored theory [20], which can be considered as colored differentials for the trivial representation $\emptyset$. Positive-colored differentials can be seen as analogs of $d_{1}$, and negativecolored differentials can be seen as analogs of $d_{-1}$.

Now, let us give a more precise definition of colored differentials, together with the explicit change of gradings in the isomorphism $(2.19)$. We note that the introduction of the fourth grading is again crucial: the $Q$-grading is indispensable for the explicit re-gradings.

Let $\Sigma$ denote the even integer such that the homology of the uncolored homology $\mathcal{H}^{\square}(K)$ with respect to the canceling differential $d_{1}$, which is onedimensional has $\left(a, q, t_{r}, t_{c}\right)$-degree $(\Sigma,-\Sigma, 0,0)$ (see [20, 77]).

Positive row-removing differentials. For any $0 \leq k<R$ there exists a "row-removing differential" $d_{R \times C \rightarrow k \times C}^{+}$(which is in fact $d_{R+k \mid 0}^{\text {col }}$ ) such that the homology of $\mathcal{H}^{R \times C}(K)$ with respect to it is isomorphic to $\mathcal{H}^{k \times C}(K)$. In particular, for $k=0$ the differential $d_{R \times C \rightarrow \emptyset}^{+}$is canceling. These differentials have the following degrees:

$$
\left(a, q, t_{r}, t_{c}\right)\left[d_{R \times C \rightarrow k \times C}^{+}\right]=(-2,2 R+2 k,-2 k-1,-1) .
$$

Since $Q=\frac{q+t_{r}-t_{c}}{R}$, we have $Q\left[d_{R \times C \rightarrow k \times C}^{+}\right]=2$. The explicit grading change in the corresponding isomorphism (2.19) is as follows: let $x$ be a generator of $\mathcal{H}^{k \times C}(K)$ with degree $\left(a, Q, t_{r}, t_{c}, q\right)$. Then the degrees of the corresponding generator $\phi(x)$ of $H^{*}\left(\mathcal{H}^{R \times C}(K), d_{R \times C \rightarrow k \times C}^{+}\right)$, denoted by $\left(\widehat{a}, \widehat{Q}, \widehat{t}_{r}, \widehat{t}_{c}, \widehat{q}\right)$, are given by

$$
\begin{aligned}
\widehat{a} & =a+C(R-k) \Sigma, \quad \widehat{Q}=Q-C(R-k) \Sigma, \\
\widehat{t}_{r} & =t_{r}+(R-k) Q+C k(R-k) \Sigma, \quad \widehat{t}_{c}=t_{c}, \\
\widehat{q} & =q-C(R-k)(R+k) \Sigma .
\end{aligned}
$$


Positive column-removing differentials. For any $0 \leq l<C$ there exists a "column-removing differential" $d_{R \times C \rightarrow R \times l}^{+}\left(\right.$which is in fact $\left.d_{R \mid l}^{\text {col }}\right)$ such that the homology of $\mathcal{H}^{R \times C}(K)$ with respect to it is isomorphic to $\mathcal{H}^{R \times l}(K)$. In particular, for $l=0$ the differential $d_{R \times C \rightarrow \emptyset}^{+}$is canceling and coincides with the canceling differential above. These differentials have the following degrees:

$$
\left(a, q, t_{r}, t_{c}\right)\left[d_{R \times C \rightarrow R \times l}^{+}\right]=(-2,2 R-2 l,-1,-2 l-1) .
$$

One can check that $Q\left[d_{R \times C \rightarrow R \times l}^{+}\right]=2$. The grading change formulas have the form

$$
\begin{aligned}
\widehat{a} & =a+R(C-l) \Sigma, \quad \widehat{Q}=Q-R(C-l) \Sigma, \\
\widehat{t_{r}} & =t_{r}, \quad \widehat{t}_{c}=t_{c}+(C-l) Q+R l(C-l) \Sigma, \\
\widehat{q} & =q+(C-l) Q+R(C-l)(l-R) \Sigma .
\end{aligned}
$$

Negative row-removing differentials. For any $0 \leq k<R$ there exists a "row-removing differential" $d_{R \times C \rightarrow k \times C}^{-}$(which is in fact $d_{k \mid C}^{\text {col }}$ ) such that the homology of $\mathcal{H}^{R \times C}(K)$ with respect to it is isomorphic to $\mathcal{H}^{k \times C}(K)$. In particular, for $k=0$ the differential $d_{R \times C \rightarrow \emptyset}^{-}$is canceling. These differentials have the following degrees:

$$
\left(a, q, t_{r}, t_{c}\right)\left[d_{R \times C \rightarrow k \times C}^{-}\right]=(-2,2 k-2 C,-2 k-2 R-1,-2 C-1) .
$$

One can check that $Q\left[d_{R \times C \rightarrow k \times C}^{-}\right]=-2$. The grading change formulas have the form

$$
\begin{aligned}
\widehat{a} & =a+C(R-k) \Sigma, \quad \widehat{Q}=Q+C(R-k) \Sigma, \\
\widehat{t_{r}} & =t_{r}+C(R-k)(R+k) \Sigma, \quad \widehat{t}_{c}=t_{c}+C^{2}(R-k) \Sigma, \\
\widehat{q} & =q+k Q+C(R-k)(C-k) \Sigma .
\end{aligned}
$$

Negative column-removing differentials. For any $0 \leq l<C$ there exists a "column-removing differential" $d_{R \times C \rightarrow R \times l}^{-}$(which is in fact $d_{0 \mid C+l}^{\text {col }}$ ) such that the homology of $\mathcal{H}^{R \times C}(K)$ with respect to it is isomorphic to $\mathcal{H}^{R \times l}(K)$. In particular, for $l=0$ the differential $d_{R \times C \rightarrow \emptyset}^{-}$is canceling and coincides with the canceling negative differential above. These differentials have the following degrees:

$$
\left(a, q, t_{r}, t_{c}\right)\left[d_{R \times C \rightarrow R \times l}^{-}\right]=(-2,-2 l-2 C,-2 R-1,-2 l-2 C-1) .
$$

One can check that $Q\left[d_{R \times C \rightarrow R \times l}^{-}\right]=-2$. The grading change formulas have the form

$$
\begin{aligned}
\widehat{a} & =a+R(C-l) \Sigma, \quad \widehat{Q}=Q+R(C-l) \Sigma, \\
\widehat{t_{r}} & =t_{r}+R^{2}(C-l) \Sigma, \quad \widehat{t}_{c}=t_{c}+R(C-l)(C+l) \Sigma, \\
\widehat{q} & =q+R(C-l)(C+l) \Sigma .
\end{aligned}
$$


To better explain such a large structure of differentials, we present them in Figure 2, This diagram represents the case of the representation labeled by the Young diagram with $R=4$ rows and $C=5$ columns. An arrow labeled by $d_{n \mid m}$ going from a Young diagram $D_{1}$ to $D_{2}$ means that there exists a differential $d_{n \mid m}^{\text {col }}$ on $\mathcal{H}^{D_{1}}(K)$ and $\mathcal{H}^{D_{2}}(K)$ such that

$$
H^{*}\left(\mathcal{H}^{D_{1}}(K), d_{n \mid m}^{\text {col }}\right) \cong \mathcal{H}^{D_{2}}(K) .
$$

There are three "special" differentials which are represented by thick lines: two of them $\left(d_{4 \mid 0}^{\text {col }}\right.$ and $\left.d_{0 \mid 5}^{\text {col }}\right)$ are canceling, i.e. the homology with respect to them is one-dimensional, and the third one (the middle one) is trivial: $d_{4 \mid 5}^{\mathrm{col}}=0$.

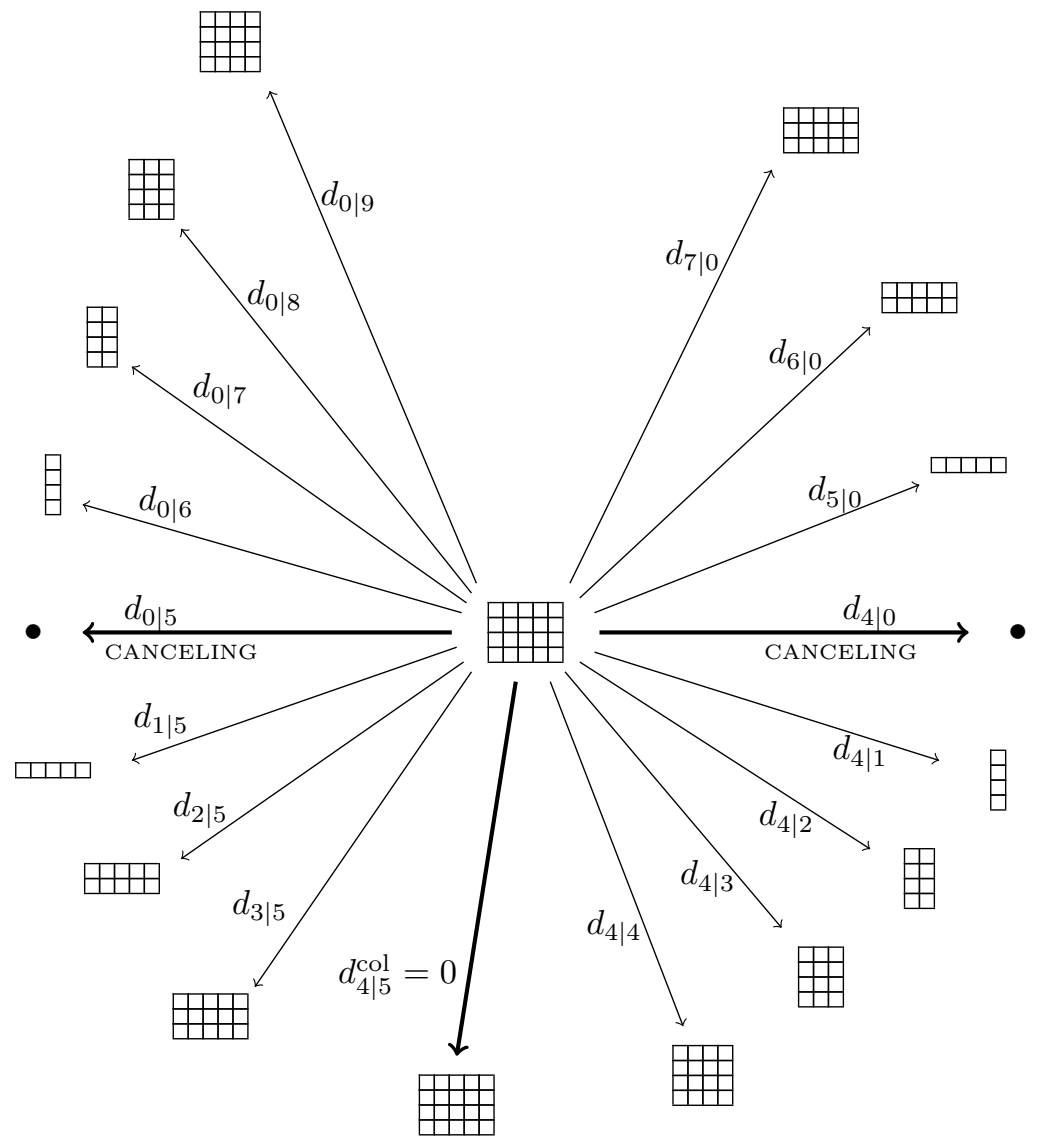

Fig. 2. Colored differentials for the $4 \times 5$ rectangle

In the general case of an arbitrary $R \times C$ rectangular Young diagram $\lambda$, the picture would be of the same form with $2 R+2 C-1$ differentials: written 
in clockwise direction, they will be $d_{R+i \mid 0}$ with $i=R-1, \ldots, 0, d_{R \mid i}$ with $i=0, \ldots, C, d_{i \mid C}$ with $i=R-1, \ldots, 0$, and $d_{0 \mid C+i}$ with $i=0, \ldots, C-1$. The canceling differentials will be $d_{R \mid 0}$ and $d_{0 \mid C}$, while the middle one would be $d_{R \mid C}\left({ }^{6}\right)$.

In the upper half of the diagram are the positive row-removing and negative column-removing differentials, with the row-removing ones $\left(d_{R+i \mid 0}\right.$ with $0 \leq i<R)$ in the upper right part, and the column-removing ones $\left(d_{0 \mid C+i}\right.$ with $0 \leq i<C$ ) in the upper left part. The negative row-removing and the positive column-removing differentials are in the lower half of the picture (below the canceling differentials). The row-removing ones $\left(d_{i \mid C}\right.$ with $0 \leq i<R)$ are in the lower left part, and the column-removing ones $\left(d_{R \mid i}\right.$ with $0 \leq i<C$ ) are in the lower right part. The positive-colored differentials are in the right half of the diagram, while the negative-colored ones are in the left half.

We conjecture that the collection of differentials is invariant under mirror symmetry and self-symmetry. Namely, let $\lambda$ be a rectangular Young diagram and let $\mu$ be obtained from $\lambda$ by removal of some rows or columns. Then

$$
\begin{gathered}
\Phi_{\lambda}\left(d_{\lambda \rightarrow \mu}^{+}(x)\right)=d_{\lambda \rightarrow \mu}^{-}\left(\Phi_{\lambda}(x)\right), \\
M_{\lambda}\left(d_{\lambda \rightarrow \mu}^{ \pm}(x)\right)=d_{\lambda^{t} \rightarrow \mu^{t}}^{ \pm}\left(M_{\lambda}(x)\right) .
\end{gathered}
$$

The involution $\Phi$ provides an isomorphism

$$
H^{*}\left(\mathcal{H}^{\lambda}(K), d_{\lambda \rightarrow \mu}^{+}(x)\right) \stackrel{\Phi}{\longrightarrow} H^{*}\left(\mathcal{H}^{\lambda}(K), d_{\lambda \rightarrow \mu}^{-}(x)\right) .
$$

2.6. Representation theory interpretation of colored differentials. The main explanation for the existence of all these positive- and negative-colored differentials with the required behavior comes from the representation theory of $\operatorname{sl}(N)$ and $\operatorname{gl}(n \mid m)$. We include the classical Lie algebra $\operatorname{sl}(N)$ in the family of Lie superalgebras $\operatorname{gl}(n \mid m)$ by setting $m=0$.

Indeed, it is well known that the irreducible polynomial representations of $\operatorname{sl}(N)$ are labeled by Young diagrams with at most $N$ rows. Moreover, some of these representations are isomorphic: namely, for every $k$ with $0 \leq k<R$, the representations labeled by $R \times C$ and $k \times C$ rectangular Young diagrams are isomorphic as $\operatorname{sl}(R+k)$ representations. This in turn implies that the doubly-graded homologies $\mathcal{H}^{\mathrm{sl}(R+k), R \times C}$ and $\mathcal{H}^{\mathrm{sl}(R+k), k \times C}$ categorifying respectively the quantum polynomial invariants $P^{\mathrm{sl}(R+k), R \times C}(q)$ and $P^{\mathrm{sl}(R+k), k \times C}(q)$ should be isomorphic. Finally, since $\mathcal{H}^{\mathrm{sl}(R+k), R \times C}$ is isomorphic to the homology of $\mathcal{H}^{R \times C}$ with respect to the differential $d_{R+k \mid 0}$, we

$\left({ }^{6}\right)$ Although this middle colored differential $d_{R \mid C}^{\mathrm{col}}$ is trivial, the $\mathrm{gl}(R \mid C)$ colored differential $d_{R \mid C}$ is in general nontrivial and is related to knot Floer homology, in the same way as the differential $d_{0}$ in the uncolored case 20. We discuss these HFK-like differentials in Section 2.10 . 
obtain the explanation of the expected behavior of positive row-removing differentials.

As for positive column-removing differentials, one should look for representations of the Lie superalgebras $\operatorname{gl}(n \mid m)$. There is an analogous theory for polynomial representations of $\operatorname{gl}(n \mid m)$ [6, 7], extending the classical case of $\operatorname{sl}(N)$ algebras. This time, the irreducible representations are labeled by Young diagrams whose $(n+1)$ st row contains at most $m$ boxes. In the case of $\operatorname{gl}(n \mid m)$ there are much more pairs of representations that are very closely related: although in general they are not necessarily isomorphic, they share many common features that are relevant for quantum knot invariants. In particular, for fixed $n$ and $m$ there are pairs of irreducible representations that have the same dimensions and the corresponding R-matrices (obtained as representations of $U_{q}(\mathrm{gl}(n \mid m))$ are the same, up to rescaling [76, 81. Therefore, the corresponding quantum polynomial knot invariants are the same (up to scaling), and the corresponding categorifications - the knot homologies - should be isomorphic. The first list of such pairs of representations, related to the column-removing differentials, is given by the fact that for every $k$ with $0 \leq k<C$, the $\operatorname{gl}(R \mid k)$ knot homologies labeled by $R \times C$ and $R \times k$ rectangular Young diagrams are isomorphic. Now one gets the homology theory corresponding to the $(\mathrm{gl}(R \mid k), R \times C)$ representations as the homology of $\mathcal{H}^{R \times C}$ with respect to $d_{R \mid k}$. Hence, the above isomorphisms of $\mathrm{gl}(R \mid k)$ representations give rise to positive column-removing differentials.

As for negative differentials, they can be obtained in the same way as positive differentials by using "dual" isomorphisms above, combined with mirror symmetry. Indeed, two Young diagrams $\lambda$ and $\mu$ yield the abovedescribed isomorphism of $\operatorname{gl}(n \mid m)$ knot homologies if and only if $\lambda^{t}$ and $\mu^{t}$ produce isomorphic $\mathrm{gl}(m \mid n)$ knot homologies. In other words,

$$
\mathcal{H}^{\mathrm{gl}(n \mid m), \lambda}(K) \cong \mathcal{H}^{\mathrm{gl}(n \mid m), \mu}(K) \Leftrightarrow \mathcal{H}^{\mathrm{gl}(m \mid n), \lambda^{t}}(K) \cong \mathcal{H}^{\mathrm{gl}(m \mid n), \mu^{t}}(K) .
$$

In such a way the existence of negative row-removing differentials $d_{k \mid C}^{\text {col }}$ with $0 \leq k<R$ is a consequence of the isomorphism of $\operatorname{gl}(k \mid C)$ homologies corresponding to $R \times C$ and $k \times C$ Young diagrams. Finally, the existence of negative column-removing differentials $d_{0 \mid C+k}^{\text {col }}$ with $0 \leq k<C$ follows from the isomorphism of $\mathrm{gl}(0 \mid C+k)$ representations corresponding to $R \times C$ and $R \times k$ Young diagrams.

2.7. Universal colored differentials. There exists yet another set of colored differentials on $\mathcal{H}^{\lambda}(K)$ such that the homology with respect to any of them is isomorphic to $\mathcal{H}^{\lambda^{\prime}}(K)$ for some $\lambda^{\prime}$ obtained from $\lambda$ by removing some rows or columns. The difference from the differentials $d_{n \mid m}^{\text {col }}$ above is that they are universal in the sense that their $a$-degree is 0 . 
Although the insights from the theory of deformations of potentials imply that such universal colored differentials should exist for any row or column removal, we have managed to find such differentials explicitly only in the cases when $\lambda$ has two rows or two columns.

ConjeCture 2.10. Let $K$ be a knot. Let $\lambda$ be a $2 \times C$ rectangular diagram and let $\lambda^{\prime}$ be $a 1 \times C$ rectangular diagram. Then there exists a differential $d^{\uparrow}$ on $\mathcal{H}^{\lambda}(K)$ of a-degree 0 such that the homology of $\mathcal{H}^{\lambda}(K)$ with respect to $d^{\uparrow}$ is isomorphic to $\mathcal{H}^{\lambda^{\prime}}(K)$.

Let $\mu$ be an $R \times 2$ rectangular diagram and let $\mu^{\prime}$ be an $R \times 1$ rectangular diagram. Then there exists a differential $d^{\leftarrow}$ on $\mathcal{H}^{\mu}(K)$ of a-degree 0 such that the homology of $\mathcal{H}^{\mu}(K)$ with respect to $d^{\leftarrow}$ is isomorphic to $\mathcal{H}^{\mu^{\prime}}(K)$.

To be more precise, we give explicit values for the degrees of the differentials $d^{\uparrow}$ and $d^{\leftarrow}$ and the degree changes in the isomorphisms of Conjecture 2.10. We describe everything in the $\left(a, Q, t_{r}, t_{c}, q\right)$-gradings. The degrees of the differentials are given by

$$
\begin{aligned}
\operatorname{deg} d^{\uparrow} & =(0,0,-2,0,2), \\
\operatorname{deg} d^{\leftarrow} & =(0,0,0,2,2) .
\end{aligned}
$$

The first isomorphism from Conjecture 2.10 gives

$$
H^{*}\left(\mathcal{H}^{\lambda}(K), d^{\uparrow}\right) \cong \mathcal{H}^{\lambda^{\prime}}(K) .
$$

Let $x$ be a generator of $\mathcal{H}^{\lambda^{\prime}}(K)$ with degree $\left(a, Q, t_{r}, t_{c}, q\right)$. Then the degrees of the corresponding generator $\phi(x)$ of $H^{*}\left(\mathcal{H}^{\lambda}(K), d^{\uparrow}\right)$, denoted by $\left(\widehat{a}, \widehat{Q}, \widehat{t}_{r}, \widehat{t_{c}}, \widehat{q}\right)$, are given by

$$
\widehat{a}=2 a, \quad \widehat{Q}=2 Q, \quad \widehat{t}_{r}=4 t_{r}, \quad \widehat{t}_{c}=2 t_{c}, \quad \widehat{q}=4 q-2 t_{c} .
$$

The second isomorphism from Conjecture 2.10 gives

$$
H^{*}\left(\mathcal{H}^{\mu}(K), d^{\leftarrow}\right) \cong \mathcal{H}^{\mu^{\prime}}(K) \text {. }
$$

Let $x$ be a generator of $\mathcal{H}^{\mu^{\prime}}(K)$ with degree $\left(a, Q, t_{r}, t_{c}, q\right)$. Then the degrees of the corresponding generator $\phi(x)$ of $H^{*}\left(\mathcal{H}^{\mu}(K), d^{\leftarrow}\right)$, denoted by $\left(\widehat{a}, \widehat{Q}, \widehat{t_{r}}, \widehat{t_{c}}, \widehat{q}\right)$, are given by

$$
\widehat{a}=2 a, \quad \widehat{Q}=2 Q, \quad \widehat{t}_{r}=2 t_{r}, \quad \widehat{t}_{c}=4 t_{c}, \quad \widehat{q}=2 q+2 t_{c} .
$$

2.8. Refined exponential growth. Now we are ready to formulate a quantitative refinement of the "exponential growth conjecture" from [44]. Throughout, $\lambda$ denotes a representation corresponding to a Young diagram of size $R \times C$.

ConjeCture 2.11. Let $K$ be a two-bridge knot or a torus knot, and let $\widetilde{\mathcal{P}}^{\lambda}(K)\left(a, Q, t_{r}\right)$ denote the Poincaré polynomial (in $\left(a, Q, t_{r}\right)$-gradings, i.e. after setting $\left.t_{c}=1\right)$ of $\widetilde{\mathcal{H}}^{\lambda}(K)$. Let $\widetilde{\mathcal{P}}^{\Lambda^{R}}(K)\left(a, Q, t_{r}\right)$ denote the Poincaré 
polynomial of $\widetilde{\mathcal{H}}^{\Lambda^{R}}(K)$ (Rth antisymmetric colored homology). Then

$$
\widetilde{\mathcal{P}}^{\lambda}(K)\left(a, Q, t_{r}\right)=\left(\widetilde{\mathcal{P}}^{\Lambda^{R}}(K)\left(a, Q, t_{r}\right)\right)^{C} .
$$

By using mirror symmetry and switching $t_{r} \leftrightarrow t_{c}$, we get the dual statement:

Conjecture 2.12. Let $K$ be a two-bridge knot or a torus knot. Let $\widetilde{\mathcal{P}}^{\lambda}(K)\left(a, Q, t_{c}\right)$ denote the Poincaré polynomial in $\left(a, Q, t_{c}\right)$-gradings of $\widetilde{\mathcal{H}}^{\lambda}(K)$, and let $\widetilde{\mathcal{P}}^{S^{C}}(K)\left(a, Q, t_{c}\right)$ denote the Poincaré polynomial of $\widetilde{\mathcal{H}}^{S^{C}}(K)$ (Cth symmetric colored homology). Then

$$
\widetilde{\mathcal{P}}^{\lambda}(K)\left(a, Q, t_{c}\right)=\left(\widetilde{\mathcal{P}}^{S^{C}}(K)\left(a, Q, t_{c}\right)\right)^{R} .
$$

COROLlary 2.13. Let $K$ be a two-bridge knot or a torus knot. The dimensions of the "rectangular homology groups" satisfy

$$
\operatorname{dim} \mathcal{H}^{\lambda}(K)=\left(\operatorname{dim} \mathcal{H}^{\square}(K)\right)^{|\lambda|} .
$$

Moreover, the similar statement holds in $(a, Q)$-gradings, i.e. with both $t_{r}$ and $t_{c}$ specialized to 1 :

$$
\widetilde{\mathcal{P}}^{\lambda}(K)(a, Q)=(\widetilde{\mathcal{P}} \square(K)(a, Q))^{|\lambda|} .
$$

Corollary 2.14. Let $K$ be a two-bridge knot or a torus knot. The Poincaré polynomial of the symmetric homology in $\left(a, Q, t_{r}\right)$-gradings can be deduced from the uncolored Poincaré polynomial (recall that $t_{r}=t_{c}=t$ for the uncolored homology):

$$
\widetilde{\mathcal{P}}^{S^{r}}(K)\left(a, Q=q, t_{r}=t, t_{c}=1\right)=\left(\mathcal{P}^{\square}(K)(a, q, t)\right)^{r} .
$$

The Poincaré polynomial of the antisymmetric homology in $\left(a, Q, t_{c}\right)$-gradings can be deduced from the uncolored Poincaré polynomial:

$$
\widetilde{\mathcal{P}}^{\Lambda^{r}}(K)\left(a, Q=q, t_{r}=1, t_{c}=t\right)=\left(\mathcal{P}^{\square}(K)(a, q, t)\right)^{r} .
$$

Going back to original $q$-degrees, i.e. the ordinary version of the colored homology $\mathcal{H}^{\lambda}(K)$, the above relations reduce to two-variable (in variables $a$ and $t$ ) exponential growths:

$$
\mathcal{P}^{S^{r}}(K)\left(a, q=1, t_{r}=t, t_{c}=1\right)=\left(\mathcal{P}^{\square}(K)(a, q=1, t)\right)^{r}
$$

and

$$
\mathcal{P}^{\Lambda^{r}}(K)\left(a, q=1, t_{r}=1, t_{c}=t\right)=\left(\mathcal{P}^{\square}(K)(a, q=1, t)\right)^{r} .
$$

Although such nice (and surprising) exponential growth properties should exist for large classes of knots, it is not expected that this should hold in general, i.e. for every knot. For example, the uncolored homology of the knot $9_{42}$ is 9 -dimensional (see 20]), its (triply-graded) $S^{2}$-colored homology 
is computed and the result has 401 generators. Therefore, we do not have any exponential growth property for it $\left(7^{7}\right)$.

Nevertheless, Wedrich [93] proved that there is a spectral sequence from $\Lambda^{r}$-colored homology to the $r$ th power of uncolored homology; see Theorem 1.3 ,

2.9. Relation to the super- $A$-polynomial. The exponential growth of the colored HOMFLYPT homology summarized in (2.30)-2.34) nicely complements the results of [30, 31, 71], where the large- $r$ limit of the $S^{r}$-homology was studied in detail. In particular, it was conjectured in [31] that for an arbitrary knot $K$ the $S^{r}$-colored superpolynomials obey a recursion relation that comes from quantization of an algebraic curve $A^{\text {super }}(x, y ; a, t)=0$. Specifically, this recursion relation has the following general form:

$$
\mathcal{P}^{S^{r+n}}(K ; a, q, t)+a_{n-1} \mathcal{P}^{S^{r+n-1}}(K ; a, q, t)+\cdots+a_{0} \mathcal{P}^{S^{r}}(K ; a, q, t)=0
$$

and can be conveniently expressed in the operator form $\widehat{A}^{\text {super }} \mathcal{P}^{S^{*}}=0$, where the coefficients $a_{i} \equiv a_{i}(x ; a, q, t)$ are rational functions of $a, q, t$, and $x \equiv q^{r}$. To make contact with (2.33), we need to set $q=1$, which, by definition, reduces the operator $\widehat{A^{\text {super }}}$ to its characteristic polynomial, the super- $A$-polynomial $A^{\text {super }}(x, y ; a, t)$.

Furthermore, since setting $q=1$ also implies $x=q^{r}=1$, we conclude that making contact with 2.33 involves comparison with the super$A$-polynomial evaluated at $x=1$. Something very nice happens in this limit: in every example studied so far, all coefficients $a_{i}(x ; a, t)$ of the super- $A$ polynomial vanish, except for $a_{n-1}$. As a result, 2.38 reduces to a much simpler recursion relation:

$$
\mathcal{P}^{S^{r+n}}(K ; a, q=1, t)+a_{n-1}(x=1, a, t) \mathcal{P}^{S^{r+n-1}}(K ; a, q=1, t)=0,
$$

which indeed takes the form 2.33). Specifically, it can be consistent with (2.33) if and only if $a_{n-1}(x=1, a, t)=\mathcal{P}(K ; a, q=1, t)$. We conjecture that this is always the case, thus providing a bridge between our present work and [30, 31, 71]:

Conjecture 2.15. For any knot $K$, the following relation between the super-A-polynomial $A^{\text {super }}(x, y ; a, t)$ and the (uncolored) superpolynomial holds:

$$
A^{\text {super }}(x=1, y ; a, t)=y^{k}+y^{k-1} \mathcal{P}(K ; a, q=1, t) .
$$

$\left({ }^{7}\right)$ This could also be seen at the level of the $S^{2}$-colored HOMFLYPT polynomial of $9_{42}$. It has nonzero terms with $a$-degree -6 , while uncolored homology is concentrated in $a$-degrees $-2,0$ and 2 . We thank Paul Wedrich for providing us with his computations of colored HOMFLYPT polynomials. 
2.10. Symmetric and antisymmetric representations. In [44 the conjectural triply-graded colored HOMFLYPT homology for the symmetric and antisymmetric representations has been computed for plenty of knots. We obtain a quadruply-graded theory by adding the $t$-degree of the corresponding generator of colored homology for the dual representation. The $t$-grading used in 44] (and called "old") corresponds to the $t_{r}$-grading in this paper. The other $t_{c}$-grading coincides with the $t$-grading used in $[2,21,20,45]$. In the case of symmetric representations, we have two additional properties of quadruply-graded colored homology: $\delta$-grading and HFK-like differential. To every generator $x$ of the $S^{r}$-colored quadruply-graded theory we can associate a $\delta$-grading by

$$
\delta(x)=a(x)+\frac{q(x)}{2}-\frac{t_{r}(x)+t_{c}(x)}{2}=a(x)+\frac{Q(x)}{2}-t_{r}(x) .
$$

We call a knot $K S^{r}$-thin if all generators of $\mathcal{H}^{S^{r}}(K)$ have the same $\delta$-grading. In the uncolored case, $r=1$, this coincides with the definition of a thin knot. Also, in the uncolored case, the $\delta$-grading of all generators of a thin knot $K$ is equal to $\Sigma / 2$, where $\Sigma$ is defined as in Section 2.5 .

Conjecture 2.16. Every thin knot $K$ is also $S^{r}$-thin with the $\delta$-grading of all generators of $\mathcal{H}^{S^{r}}(K)$ being equal to $r \Sigma / 2$.

Additionally, the refined exponential growth conjecture (2.34) holds for all thin knots.

The action of the "special" differential $d_{1 \mid r}$ on $S^{r}$-colored homology is related to the action of the differential $d_{1 \mid 1}$ on uncolored homology. The latter is denoted $d_{0}$ in [20], and it is conjectured that the homology $H^{*}\left(\mathcal{H}^{\square}(K), d_{0}\right)$ is isomorphic to the knot Floer homology of $K$.

Conjecture 2.17. Let $\widetilde{\mathcal{P}}_{\mathrm{HFK}}^{S^{r}}(K)\left(a, Q, t_{r}, t_{c}\right)$ denote the Poincaré polynomial in $\left(a, Q, t_{r}, t_{c}\right)$-gradings of $H^{*}\left(\widetilde{\mathcal{H}}^{S^{r}}(K), d_{1 \mid r}\right)$. Let $\widetilde{\mathcal{P}}_{\mathrm{HFK}}(K)(a, q, t)$ denote the Poincaré polynomial of $H^{*}\left(\mathcal{H}^{\square}(K), d_{1 \mid 1}\right)$. Then

$$
\widetilde{\mathcal{P}}_{\mathrm{HFK}}^{S^{r}}(K)\left(a, Q=q, t_{r}=t, t_{c}=1\right)=\left(\widetilde{\mathcal{P}}_{\mathrm{HFK}}(a, q, t)\right)^{r} .
$$

We note two things: first, the above property does not descend to any relation at the colored HOMFLYPT polynomial level, due to a nontrivial presence of the $Q$-grading. Secondly, both $d_{1 \mid 1}$ on $\mathcal{H}^{\square}(K)$ and $d_{1 \mid r}$ on $\mathcal{H}^{S^{r}}(K)$ have nonzero $\delta$-grading and therefore act trivially on thin knots. Hence, the relation (2.42) in the case of thin knots becomes simply (2.34). However, once we pass to thick knots, in general the two relations are different, as we shall show in the example below for the $(3,4)$-torus knot.

3. Examples. In this section we give some models of quadruply-graded colored HOMFLYPT homology that exhibit all of the structural properties 
and satisfy all of the conjectures from the previous sections. This gives a highly nontrivial check of the existence and consistency of such large and beautiful structures in colored HOMFLYPT homology. Also, we note that the three-variable $\left(a, q, t_{c}\right)$-specializations of the expressions that we get for the superpolynomials match other existing results that were computed by different methods [2, 14, 21].

3.1. Trefoil. We start with the trefoil and its $S^{2}$ - and $\Lambda^{2}$-colored homologies. The Poincaré polynomial of the uncolored HOMFLYPT homology of the trefoil is $(20])$

$$
\mathcal{P}^{\square}\left(3_{1}\right)(a, q, t)=a^{2} q^{-2}+a^{2} q^{2} t^{2}+a^{4} t^{3} .
$$

The uncolored homology is the only one where the two $t$-gradings coincide, i.e.

$$
\mathcal{P}^{\square}\left(3_{1}\right)\left(a, q, t_{r}, t_{c}\right)=\mathcal{P}^{\square}\left(3_{1}\right)\left(a, q, t=t_{r} t_{c}\right) .
$$

From (3.1) we can get the value of the $S$-invariant of the trefoil (which we denote $\Sigma$ in this paper):

$$
\Sigma\left(3_{1}\right)=2 .
$$

For easier visualization, triply-graded homologies are usually presented in diagram form. A generator of $(a, q, t)$-degree $(i, j, k)$ is presented as a dot in the $(q, a)$-plane at position $(j, i)$ and it is labeled by its $t$-degree $k$. The triply-graded homology of the trefoil is presented in Figure 3 (left).
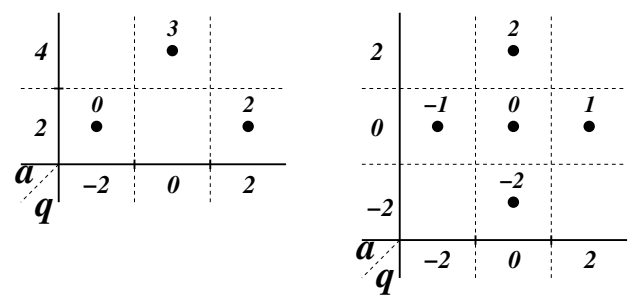

Fig. 3. The uncolored homologies of the trefoil (left) and figure-eight knot (right)

The (reduced) $\square$-colored HOMFLYPT polynomial of the trefoil knot is $P^{\square}\left(3_{1}\right)(a, q)=a^{4} q^{-4}+a^{4} q^{2}+a^{4} q^{4}+a^{4} q^{8}-a^{6}-a^{6} q^{2}-a^{6} q^{6}-a^{6} q^{8}+a^{8} q^{6}$. The value of the four-variable $\square$-colored superpolynomial (the Poincaré polynomial of the quadruply-graded $\left.\mathcal{H}^{\square}\left(3_{1}\right)\right)$ of the trefoil is

$$
\begin{aligned}
\mathcal{P} \square\left(3_{1}\right)\left(a, q, t_{r}, t_{c}\right) & =a^{4}\left(q^{-4}+q^{2} t_{r}^{2} t_{c}^{4}+q^{4} t_{r}^{2} t_{c}^{6}+q^{8} t_{r}^{4} t_{c}^{8}\right) \\
& +a^{6}\left(t_{r}^{3} t_{c}^{5}+q^{2} t_{r}^{3} t_{c}^{7}+q^{6} t_{r}^{5} t_{c}^{9}+q^{8} t_{r}^{5} t_{c}^{11}\right)+a^{8} q^{6} t_{r}^{6} t_{c}^{12} .
\end{aligned}
$$

The triply-graded $S^{2}$-homology of the trefoil, computed in [44], is obtained from (3.3) by setting $t_{c}=1$, whereas the homology obtained in [2] or [21] is obtained after setting $t_{r}=1$. 
For the second-symmetric representation the $Q$-grading is expressed as $Q=q+t_{r}-t_{c}$. Then the tilde-version, i.e. the quadruply-graded $\square$-homology in $\left(a, Q, t_{r}, t_{c}\right)$-gradings is given by

$$
\begin{aligned}
& \widetilde{\mathcal{P}}^{\square}\left(3_{1}\right)\left(a, Q, t_{r}, t_{c}\right)=a^{4}\left(Q^{-4}+t_{r}^{2} t_{c}^{4}+t_{r}^{2} t_{c}^{6}+Q^{4} t_{r}^{4} t_{c}^{8}\right) \\
& +a^{6}\left(Q^{-2} t_{r}^{3} t_{c}^{5}+Q^{-2} t_{r}^{3} t_{c}^{7}+Q^{2} t_{r}^{5} t_{c}^{9}+Q^{2} t_{r}^{5} t_{c}^{11}\right)+a^{8} t_{r}^{6} t_{c}^{12} .
\end{aligned}
$$

We note that all generators have the same $\delta$-grading, equal to +2 . Moreover, the last homology is self-symmetric: in terms of the Poincare polynomial this amounts to

$$
\widetilde{\mathcal{P}}^{\square}\left(3_{1}\right)\left(a, Q^{-1} t_{r}^{-1} t_{c}^{-2}, t_{r}, t_{c}\right)=\widetilde{\mathcal{P}} \square\left(3_{1}\right)\left(a, Q, t_{r}, t_{c}\right) .
$$

We represent the quadruply-graded homologies in diagram form in the following way: for each generator we place a dot in the $(q, a)$-plane. If the $(q, a)$-degree of a generator $x$ is $(i, j)$ then we put a dot at the point with coordinates $(i, j)$. The two $t$-degrees are drawn as labels near the dot: the $t_{r}$-grading is written above the dot (and colored light green), while the $t_{c}$-grading of a generator is written below the corresponding dot. In such a way, the homology $\mathcal{H}^{\square}\left(3_{1}\right)$ is presented in Figure 4 .

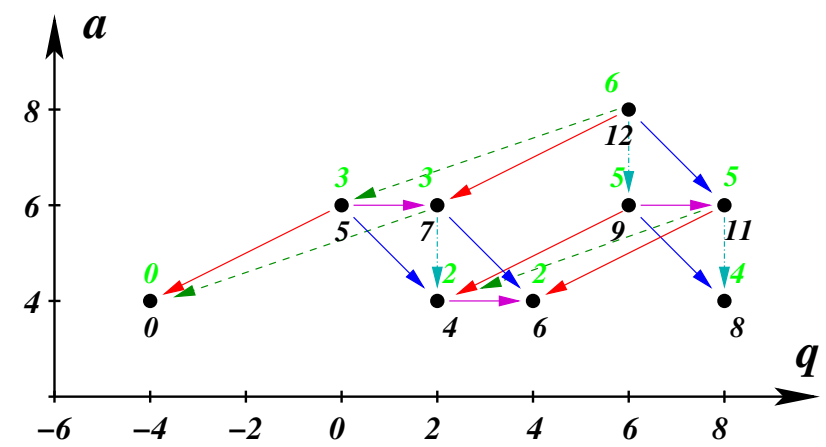

Fig. 4. The $S^{2}$-colored homology of the trefoil knot

We can see all five nontrivial colored differentials on this diagram: There are two canceling ones $d_{1 \mid 0}^{\text {col }}$ and $d_{0 \mid 2}^{\text {col }}$, represented by blue and red arrows, respectively, both leaving a single generator. Furthermore the two gl $(n \mid m)$ colored differentials $d_{1 \mid 1}^{\text {col }}$ (dash-and-dot arrows) and $d_{0 \mid 3}^{\text {col }}$ (dashed arrows), both leaving the homology isomorphic to $\mathcal{H}^{\square}\left(3_{1}\right)$. And finally the universal one $d^{\leftarrow}$ (magenta arrows), also leaving the homology isomorphic to $\mathcal{H}^{\square}\left(3_{1}\right)$. We note that the re-gradings are exactly as predicted in Sections 2.5 and 2.7 .

The diagram for the tilde-version of the homology $\widetilde{\mathcal{H}} \square\left(3_{1}\right)$ is shown in Figure 5. It is drawn analogously, with generators depicted by dots in the $(Q, a)$-plane. The refined exponential growth indeed holds:

$$
\widetilde{\mathcal{P}}^{\square}\left(3_{1}\right)\left(a, Q=q, t_{r}=t, t_{c}=1\right)=\left(\mathcal{P}^{\square}\left(3_{1}\right)(a, q, t)\right)^{2} .
$$




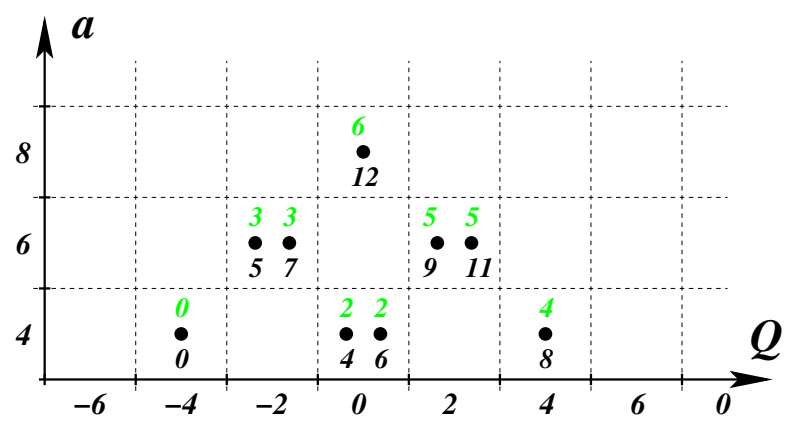

Fig. 5. The tilde-version of the $S^{2}$-colored homology of the trefoil knot $\left(Q=q+t_{r}-t_{c}\right)$

As for the second-antisymmetric representation, we see that the $\Lambda^{2}$-colored HOMFLYPT polynomial of the trefoil is given by

$$
\begin{aligned}
P^{\Xi}\left(3_{1}\right)(a, q)= & a^{4}\left(q^{-8}+q^{-4}+q^{-2}+q^{4}\right) \\
& -a^{6}\left(q^{-8}+q^{-6}+q^{-2}+1\right)+a^{8} q^{-6} .
\end{aligned}
$$

The Poincaré polynomial of the quadruply-graded homology $\mathcal{H}^{\boxminus}\left(3_{1}\right)$ equals

$$
\begin{aligned}
\mathcal{P}^{\Xi}\left(3_{1}\right)\left(a, q, t_{r}, t_{c}\right)=a^{4}\left(q^{-8}+q^{-4} t_{r}^{6} t_{c}^{2}+q^{-2} t_{r}^{4} t_{c}^{2}+q^{4} t_{r}^{8} t_{c}^{4}\right) \\
+a^{6}\left(q^{-8} t_{r}^{7} t_{c}^{3}+q^{-6} t_{r}^{5} t_{c}^{3}+q^{-2} t_{r}^{11} t_{c}^{5}+t_{r}^{9} t_{c}^{5}\right)+a^{8} q^{-6} t_{r}^{12} t_{c}^{6} .
\end{aligned}
$$

The corresponding diagram, together with the colored differentials, is presented in Figure 6. By comparing Figures 4 and 6 one can immediately see

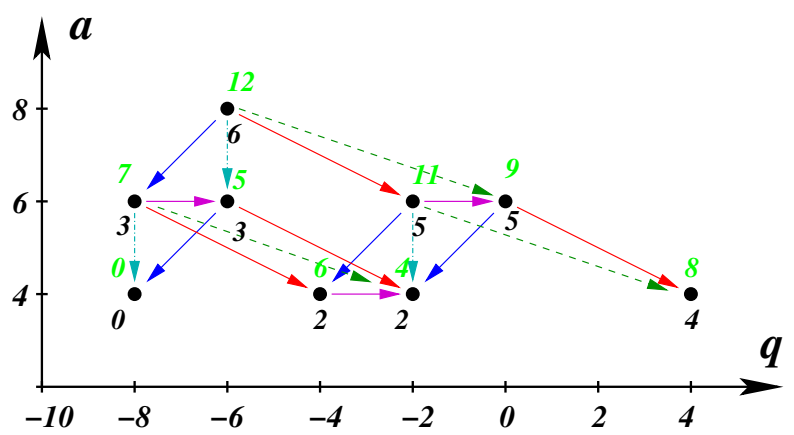

Fig. 6. The $\Lambda^{2}$-colored homology of the trefoil knot

mirror symmetry (2.11). This symmetry sends $q \mapsto-q$, and commutes with the action of the colored differentials, as can be seen from the diagrams. We remark that all re-gradings from the isomorphisms induced by colored differentials are exactly as predicted in Sections 2.5 and 2.7 .

Mirror symmetry is even better seen on the tilde-version of the homology. For the second-antisymmetric representation $\boxminus$, the $Q$-grading is obtained by 
$Q=\left(q+t_{r}-t_{c}\right) / 2$, and so

$$
\begin{aligned}
\widetilde{\mathcal{P}} \boxminus\left(3_{1}\right)(a, Q, & \left.t_{r}, t_{c}\right)=a^{4}\left(Q^{-4}+t_{r}^{6} t_{c}^{2}+t_{r}^{4} t_{c}^{2}+Q^{4} t_{r}^{8} t_{c}^{4}\right) \\
& +a^{6}\left(Q^{-2} t_{r}^{7} t_{c}^{3}+Q^{-2} t_{r}^{5} t_{c}^{3}+Q^{2} t_{r}^{11} t_{c}^{5}+Q^{2} t_{r}^{9} t_{c}^{5}\right)+a^{8} t_{r}^{12} t_{c}^{6} .
\end{aligned}
$$

Its diagram is given in Figure 7 .

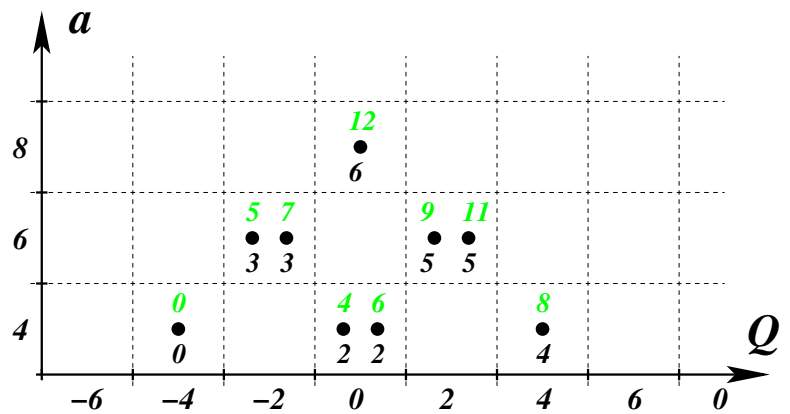

Fig. 7. The tilde-version of the $\Lambda^{2}$-colored homology of the trefoil $\operatorname{knot}(Q=$ $\left.\left(q+t_{r}-t_{c}\right) / 2\right)$

Comparison of Figures 5 and 7 shows that the two are related by a simple exchange of the $t_{r}$ - and $t_{c}$-gradings, as predicted by mirror symmetry 2.10 . Also, one can easily check that the explicit form of the other mirror symmetry 2.11 is satisfied, too.

Before passing to further examples, we note that with the explicit mirror symmetries, whenever we know the quadruply-graded $\lambda$-colored homology of a knot $K$, then we automatically also have its $\lambda^{t}$-colored homology:

$$
\widetilde{\mathcal{P}}^{\lambda^{t}}(K)\left(a, Q, t_{r}, t_{c}\right)=\widetilde{\mathcal{P}}^{\lambda}(K)\left(a, Q, t_{c}, t_{r}\right) .
$$

Therefore, in the remaining examples we present the results only for one diagram for each pair $\left(\lambda, \lambda^{t}\right)$.

3.2. Figure-eight knot. We emphasize that our conjectures and predicted structures are for all knots, and not just torus knots. In particular, all homologies conjectured in [44] (that includes $S^{2}$-colored homologies of all prime knots with up to six crossings) can be extended to quadruply-graded homologies which have all of the required properties. Here we present explicit expressions for the conjectural $S^{2}$-colored homology of the figure-eight knot. The uncolored homology of the figure-eight knot is given by

$$
\mathcal{P}^{\square}\left(4_{1}\right)(a, q, t)=a^{2} t^{2}+\left(q^{-2} t^{-1}+1+q^{2} t\right)+a^{-2} t^{-2},
$$

and its $S$-invariant is $\Sigma\left(4_{1}\right)=0$. The triply-graded homology of the figureeight knot is presented in Figure 3 (right). The $\square$-colored HOMFLYPT 
polynomial of the figure-eight knot equals

$$
\begin{aligned}
P^{\square}\left(4_{1}\right)(a, q)= & a^{4} q^{4}+a^{2}\left(-q^{-2}+q^{2}-q^{4}-q^{6}\right)+\left(q^{-6}-q^{-4}+3-q^{4}+q^{6}\right) \\
& +a^{-2}\left(-q^{-6}-q^{-4}+q^{-2}-q^{2}\right)+a^{-4} q^{-4} .
\end{aligned}
$$

The quadruply-graded $\square$-colored HOMFLYPT homology of the figure-eight knot that we computed is given by

$$
\begin{aligned}
\mathcal{P}^{\square} & \left(4_{1}\right)\left(a, q, t_{r}, t_{c}\right)=a^{4} q^{4} t_{r}^{4} t_{c}^{8}+a^{2}\left(q^{-2} t_{r} t_{c}+t_{r} t_{c}^{3}+t_{r}^{2} t_{c}^{2}+q^{2} t_{r}^{2} t_{c}^{4}+q^{4} t_{r}^{3} t_{c}^{5}+q^{6} t_{r}^{3} t_{c}^{7}\right) \\
& +\left(q^{-6} t_{r}^{-2} t_{c}^{-4}+q^{-4} t_{r}^{-1} t_{c}^{-3}+q^{-2} t_{r}^{-1} t_{c}^{-1}+q^{-2} t_{c}^{-2}+3+q^{2} t_{c}^{2}+q^{2} t_{r} t_{c}+q^{4} t_{r} t_{c}^{3}+q^{6} t_{r}^{2} t_{c}^{4}\right) \\
& +a^{-2}\left(q^{-6} t_{r}^{-3} t_{c}^{-7}+q^{-4} t_{r}^{-3} t_{c}^{-5}+q^{-2} t_{r}^{-2} t_{c}^{-4}+t_{r}^{-2} t_{c}^{-2}+t_{r}^{-1} t_{c}^{-3}+q^{2} t_{r}^{-1} t_{c}^{-1}\right)+a^{-4} q^{-4} t_{r}^{-4} t_{c}^{-8} .
\end{aligned}
$$

It is presented in diagram form in Figure 8. All generators have the same

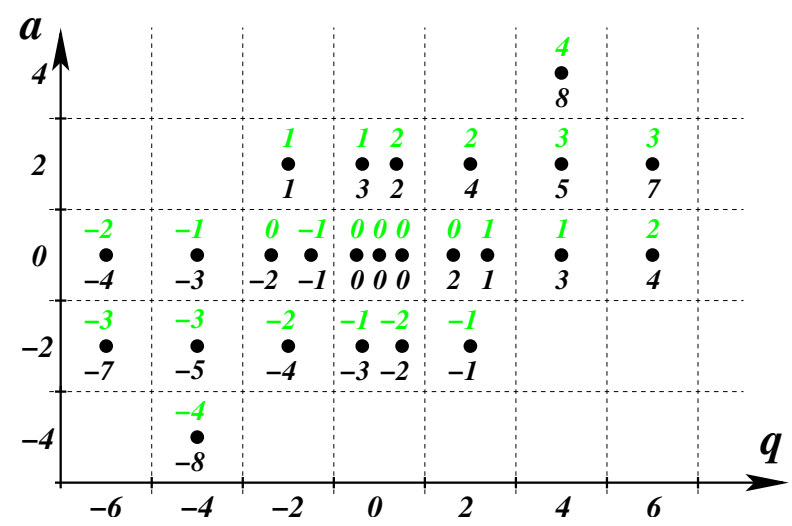

Fig. 8. The $S^{2}$-colored homology of the figure-eight knot

$\delta$-degree 0 , in accordance with the fact that $4_{1}$ is a thin knot. The homology $\mathcal{H}^{\square}\left(4_{1}\right)$ has five colored differentials: two canceling ones $d_{1 \mid 0}^{\text {col }}$ and $d_{0 \mid 2}^{\text {col }}$, two

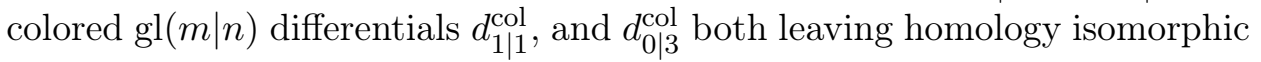
to $\mathcal{H}^{\square}\left(4_{1}\right)$, and the universal one $d^{\leftarrow}$ also leaving homology isomorphic to $\mathcal{H}^{\square}\left(4_{1}\right)$. All re-gradings are as given in Sections 2.5 and 2.7 .

The $Q$-grading is given by $Q=q+t_{r}-t_{c}$. So, the tilde-version of the $\square$-colored HOMFLYPT homology of the figure-eight knot becomes

$$
\begin{aligned}
\widetilde{\mathcal{P}}^{\square}\left(4_{1}\right) & \left(a, Q, t_{r}, t_{c}\right)=a^{4} t_{r}^{4} t_{c}^{8}+a^{2}\left(Q^{-2} t_{r} t_{c}+Q^{-2} t_{r} t_{c}^{3}+t_{r}^{2} t_{c}^{2}+t_{r}^{2} t_{c}^{4}+Q^{2} t_{r}^{3} t_{c}^{5}+Q^{2} t_{r}^{3} t_{c}^{7}\right) \\
& +\left(Q^{-4} t_{r}^{-2} t_{c}^{-4}+Q^{-2} t_{r}^{-1} t_{c}^{-3}+Q^{-2} t_{r}^{-1} t_{c}^{-1}+t_{c}^{-2}+3+t_{c}^{2}+Q^{2} t_{r} t_{c}+Q^{2} t_{r} t_{c}^{3}+Q^{4} t_{r}^{2} t_{c}^{4}\right) \\
& +a^{-2}\left(Q^{-2} t_{r}^{-3} t_{c}^{-7}+Q^{-2} t_{r}^{-3} t_{c}^{-5}+t_{r}^{-2} t_{c}^{-4}+t_{r}^{-2} t_{c}^{-2}+Q^{2} t_{r}^{-1} t_{c}^{-3}+Q^{2} t_{r}^{-1} t_{c}^{-1}\right)+a^{-4} t_{r}^{-4} t_{c}^{-8} .
\end{aligned}
$$

The tilde-version is presented in Figure 9 in $\left(a, Q, t_{r}, t_{c}\right)$-gradings. It indeed satisfies the self-symmetry 2.8 and the refined exponential growth 2.34).

3.3. (3, 4)-torus knot. In this section we describe the conjectural quadruply-graded $S^{2}$-colored homology of the $(3,4)$-torus knot $T_{3,4}$ (i.e. $8_{19}$ knot) and show that it has all the desired properties. This knot is a thick knot, 


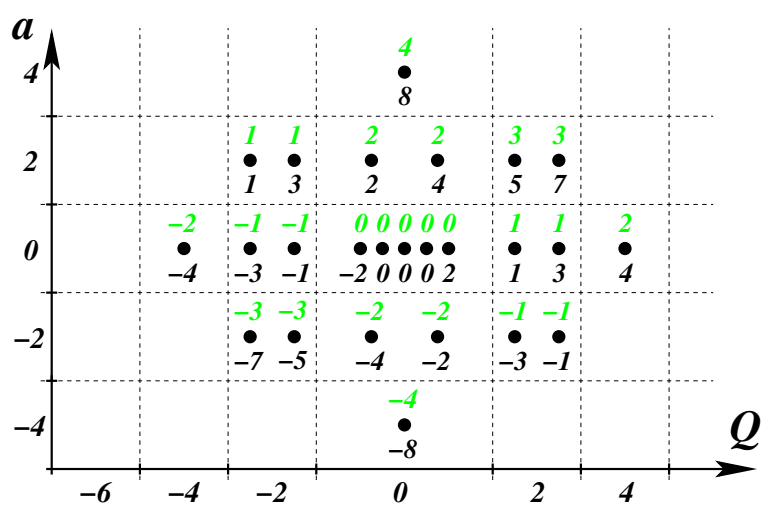

Fig. 9. The tilde-version of the $S^{2}$-colored homology of the figure-eight knot

and has nontrivial differential $d_{1 \mid 1}$ (i.e. $d_{0}$ ) on the uncolored homology, which makes this a highly nontrivial consistency check of all predicted properties. The conjectural uncolored homology of $T_{3,4}$ is given by (see [20])

$$
\begin{aligned}
\mathcal{P}^{\square}\left(T_{3,4}\right)(a, q, t)= & a^{6} q^{-6}+a^{8} q^{-4} t^{3}+a^{6} q^{-2} t^{2}+a^{8} t^{5}+a^{6} q^{2} t^{4}+a^{8} q^{4} t^{7} \\
& +a^{6} q^{6} t^{6}+a^{10} t^{8}+a^{8} q^{-2} t^{5}+a^{8} q^{2} t^{7}+a^{6} t^{4} .
\end{aligned}
$$

Its $S$-invariant is $\Sigma\left(T_{3,4}\right)=6$. There is a nontrivial differential $d_{0}$ (or $\left.d_{1 \mid 1}\right)$ on $\mathcal{H}^{\square}\left(T_{3,4}\right)$ of $(a, q, t)$-degree $(-2,0,-3)$ leaving 5 -dimensional homology isomorphic to the knot Floer homology of $T_{3,4}$ :

$$
\left(\mathcal{H}^{\square}\left(T_{3,4}\right), d_{1 \mid 1}\right)=a^{6} q^{-6}+a^{8} q^{-4} t^{3}+a^{6} t^{4}+a^{8} q^{4} t^{7}+a^{6} q^{6} t^{6} .
$$

The quadruply-graded $\square$-colored homology of $T_{3,4}$ that we have computed has 121 generators. The Poincaré polynomial of the tilde-version $\widetilde{\mathcal{H}}^{\square}\left(T_{3,4}\right)$ in $\left(a, Q, t_{r}, t_{c}\right)$ variables is given by

$$
\begin{array}{r}
\widetilde{\mathcal{P}}^{\square}\left(T_{3,4}\right)\left(a, Q, t_{r}, t_{c}\right)=a^{12}\left(Q^{-12}+Q^{-8} t_{r}^{2}\left(t_{c}^{4}+t_{c}^{6}\right)+Q^{-6} t_{r}^{4}\left(t_{c}^{8}+t_{c}^{10}\right)\right. \\
+Q^{-4} t_{r}^{4}\left(t_{c}^{8}+t_{c}^{10}+t_{c}^{12}\right)+Q^{-2} t_{r}^{6}\left(t_{c}^{12}+t_{c}^{14}\right)+t_{r}^{6}\left(t_{c}^{12}+t_{c}^{14}+t_{c}^{16}+t_{c}^{18}\right)+t_{r}^{8} t_{c}^{16} \\
\left.+Q^{2} t_{r}^{8}\left(t_{c}^{16}+t_{c}^{18}\right)+Q^{4} t_{r}^{8}\left(t_{c}^{16}+t_{c}^{18}+t_{c}^{20}\right)+Q^{6} t_{r}^{10}\left(t_{c}^{20}+t_{c}^{22}\right)+Q^{8} t_{r}^{10}\left(t_{c}^{20}+t_{c}^{22}\right)+Q^{12} t_{r}^{12} t_{c}^{24}\right) \\
+a^{14}\left(Q^{-10} t_{r}^{3}\left(t_{c}^{5}+t_{c}^{7}\right)+Q^{-8} t_{r}^{5}\left(t_{c}^{9}+t_{c}^{11}\right)+Q^{-6} t_{r}^{5}\left(t_{c}^{9}+2 t_{c}^{11}+t_{c}^{13}\right)\right. \\
+Q^{-4} t_{r}^{7}\left(2 t_{c}^{13}+3 t_{c}^{15}+t_{c}^{17}\right)+Q^{-2} t_{r}^{7}\left(t_{c}^{13}+2 t_{c}^{15}+2 t_{c}^{17}+t_{c}^{19}\right)+Q^{-2} t_{r}^{9}\left(t_{c}^{17}+t_{c}^{19}\right) \\
+t_{r}^{9}\left(2 t_{c}^{17}+3 t_{c}^{19}+t_{c}^{21}\right)+Q^{2} t_{r}^{9}\left(t_{c}^{17}+2 t_{c}^{19}+2 t_{c}^{21}+t_{c}^{23}\right)+Q^{2} t_{r}^{11}\left(t_{c}^{21}+t_{c}^{23}\right) \\
+Q^{4} t_{r}^{11}\left(2 t_{c}^{21}+3 t_{c}^{23}+t_{c}^{25}\right)+Q^{6} t_{r}^{11}\left(t_{c}^{21}+2 t_{c}^{23}+t_{c}^{25}\right)+Q^{8} t_{r}^{13}\left(t_{c}^{25}+t_{c}^{27}\right) \\
\left.+Q^{10} t_{r}^{13}\left(t_{c}^{25}+t_{c}^{27}\right)\right)+a^{16}\left(Q^{-8} t_{r}^{6} t_{c}^{12}+Q^{-6} t_{r}^{8}\left(t_{c}^{14}+2 t_{c}^{16}+t_{c}^{18}\right)+Q^{-4} t_{r}^{8}\left(t_{c}^{16}+t_{c}^{18}\right)\right. \\
+Q^{-4} t_{r}^{10} t_{c}^{20}+Q^{-2} t_{r}^{10}\left(t_{c}^{18}+3 t_{c}^{20}+2 t_{c}^{22}\right)+t_{r}^{10}\left(t_{c}^{20}+t_{c}^{22}+t_{c}^{24}\right)+t_{r}^{12}\left(t_{c}^{22}+2 t_{c}^{24}+t_{c}^{26}\right) \\
+Q^{2} t_{r}^{12}\left(t_{c}^{22}+3 t_{c}^{24}+2 t_{c}^{26}\right)+Q^{4} t_{r}^{12}\left(t_{c}^{24}+t_{c}^{26}\right)+Q^{4} t_{r}^{14} t_{c}^{28}+Q^{6} t_{r}^{14}\left(t_{c}^{26}+2 t_{c}^{28}+t_{c}^{30}\right) \\
\left.+Q^{8} t_{r}^{14} t_{c}^{28}\right)+a^{18}\left(Q^{-4} t_{r}^{11}\left(t_{c}^{21}+t_{c}^{23}\right)+Q^{-2} t_{r}^{13}\left(t_{c}^{25}+t_{c}^{27}\right)+t_{r}^{13}\left(t_{c}^{25}+t_{c}^{27}\right)\right. \\
\left.+Q^{2} t_{r}^{15}\left(t_{c}^{29}+t_{c}^{31}\right)+Q^{4} t_{r}^{15}\left(t_{c}^{29}+t_{c}^{31}\right)\right)+a^{20} t_{r}^{16} t_{c}^{32} .
\end{array}
$$


The Poincaré polynomial in $\left(a, q, t_{r}, t_{c}\right)$-gradings can be obtained from the above expression by

$$
\mathcal{P}^{\square}\left(T_{3,4}\right)\left(a, q, t_{r}, t_{c}\right)=\widetilde{\mathcal{P}}^{\square}\left(T_{3,4}\right)\left(a, Q=q, t_{r}=t_{r} q^{-1}, t_{c}=t_{c} q\right) .
$$

This homology has all the expected properties: there are four colored $\operatorname{gl}(n \mid m)$ differentials $d_{1 \mid 0}^{\text {col }}, d_{0 \mid 2}^{\text {col }}, d_{1 \mid 1}^{\text {col }}$ and $d_{0 \mid 3}^{\text {col }}$, with the properties and re-gradings exactly matching the predicted behavior from Section 2.5. Moreover, there exists a universal colored differential $d^{\leftarrow}$ as predicted in Section 2.7. Furthermore, the homology $\widetilde{\mathcal{H}} \square\left(T_{3,4}\right)$ has the self-symmetry property

$$
\widetilde{\mathcal{P}} \square\left(T_{3,4}\right)\left(a, Q^{-1} t_{r}^{-1} t_{c}^{-2}, t_{r}, t_{c}\right)=\widetilde{\mathcal{P}} \square\left(T_{3,4}\right)\left(a, Q, t_{r}, t_{c}\right) .
$$

It also satisfies the refined exponential growth property

$$
\widetilde{\mathcal{P}}^{\square}\left(T_{3,4}\right)\left(a, Q=q, t_{r}=t, t_{c}=1\right)=\left(\mathcal{P}^{\square}\left(T_{3,4}\right)(a, q, t)\right)^{2} .
$$

Moreover, since the HFK differential $d_{0}$ on $\mathcal{H}^{\square}\left(T_{3,4}\right)$ is nontrivial, this time the HFK-like exponential growth property (2.42) gives a new relation on the homologies. Indeed, there exists a differential $d_{1 \mid 2}$ on $\widetilde{\mathcal{H}} \square\left(T_{3,4}\right)$ of $\left(a, Q, t_{r}, t_{c}\right)$-degree $(-2,0,-3,-5)$, so that the homology $H^{*}\left(\widetilde{\mathcal{H}}^{\square}\left(T_{3,4}\right), d_{1 \mid 2}\right)$ is 25-dimensional with Poincaré polynomial

$$
\begin{array}{r}
\left(\widetilde{\mathcal{H}}^{\boxplus}\left(T_{3,4}\right), d_{1 \mid 2}\right)\left(a, Q, t_{r}, t_{c}\right)=a^{12}\left(Q^{-12}+Q^{-6} t_{r}^{4}\left(t_{c}^{8}+t_{c}^{10}\right)+t_{r}^{6}\left(t_{c}^{16}+t_{c}^{18}\right)+t_{r}^{8} t_{c}^{16}\right. \\
\left.+Q^{6} t_{r}^{10}\left(t_{c}^{20}+t_{c}^{22}\right)+Q^{12} t_{r}^{12} t_{c}^{24}\right)+a^{14}\left(Q^{-10} t_{r}^{3}\left(t_{c}^{5}+t_{c}^{7}\right)+Q^{-4} t_{r}^{7}\left(t_{c}^{13}+t_{c}^{15}\right)\right. \\
\left.+Q^{-2} t_{r}^{7}\left(t_{c}^{17}+t_{c}^{19}\right)+Q^{2} t_{r}^{9}\left(t_{c}^{21}+t_{c}^{23}\right)+Q^{4} t_{r}^{11}\left(t_{c}^{21}+t_{c}^{23}\right)+Q^{10} t_{r}^{13}\left(t_{c}^{25}+t_{c}^{27}\right)\right) \\
+a^{16}\left(Q^{-8} t_{r}^{6} t_{c}^{12}+t_{r}^{10}\left(t_{c}^{22}+t_{c}^{24}\right)+Q^{8} t_{r}^{14} t_{c}^{28}\right)
\end{array}
$$

and such that

$$
\left(\widetilde{\mathcal{H}}^{\square}\left(T_{3,4}\right), d_{1 \mid 2}\right)\left(a, Q=q, t_{r}=t, t_{c}=1\right)=\left(\left(\mathcal{H}^{\square}\left(T_{3,4}\right), d_{1 \mid 1}\right)(a, q, t)\right)^{2} .
$$

3.4. (2,2)-representation. In this section we describe the $\boxplus$-colored HOMFLYPT homology of the trefoil knot. It should categorify the reduced $\boxplus$-colored HOMFLYPT polynomial of the trefoil:

$$
\begin{aligned}
& P^{\boxplus}\left(3_{1}\right)(a, q)=a^{16}+a^{14}\left(-q^{-6}-2 q^{-4}-q^{-2}-q^{2}-2 q^{4}-q^{6}\right) \\
& +a^{12}\left(4+2 q^{-10}+2 q^{-8}+2 q^{-6}+q^{-4}+3 q^{-2}+3 q^{2}+q^{4}+2 q^{6}+2 q^{8}+2 q^{10}\right) \\
& \quad+a^{10}\left(-2-q^{-14}-2 q^{-12}-q^{-10}-q^{-8}-3 q^{-6}\right. \\
& \left.-4 q^{-4}-3 q^{-2}-3 q^{2}-4 q^{4}-3 q^{6}-q^{8}-q^{10}-2 q^{12}-q^{14}\right) \\
& +a^{8}\left(2+q^{-16}+q^{-10}+2 q^{-8}+q^{-6}+q^{-4}+q^{-2}+q^{2}+q^{4}+q^{6}+2 q^{8}+q^{10}+q^{16}\right) .
\end{aligned}
$$

The $\boxplus$-colored HOMFLYPT homology of the trefoil knot that we computed has 81 generators. Its four-variable Poincaré polynomial is given by

$$
\begin{aligned}
\mathcal{P} \boxplus\left(3_{1}\right)\left(a, q, t_{r}, t_{c}\right)=a^{16} t_{r}^{24} t_{c}^{24}+a^{14}\left(q^{-6} t_{r}^{19} t_{c}^{17}+q^{-4}\left(t_{r}^{17} t_{c}^{17}+t_{r}^{19} t_{c}^{19}\right)\right. \\
\left.+q^{-2} t_{r}^{17} t_{c}^{19}+q^{2} t_{r}^{23} t_{c}^{21}+q^{4}\left(t_{r}^{21} t_{c}^{21}+t_{r}^{23} t_{c}^{23}\right)+q^{6} t_{r}^{21} t_{c}^{23}\right)
\end{aligned}
$$




$$
\begin{array}{r}
+a^{12}\left(q^{-10}\left(t_{r}^{12} t_{c}^{10}+t_{r}^{14} t_{c}^{12}\right)+2 q^{-8} t_{r}^{12} t_{c}^{12}+q^{-6}\left(t_{r}^{10} t_{c}^{12}+t_{r}^{12} t_{c}^{14}\right)+q^{-4} t_{r}^{18} t_{c}^{14}\right. \\
+q^{-2}\left(t_{r}^{16} t_{c}^{14}+2 t_{r}^{18} t_{c}^{16}\right)+\left(3 t_{r}^{16} t_{c}^{16}+t_{r}^{18} t_{c}^{18}\right)+q^{2}\left(t_{r}^{14} t_{c}^{16}+2 t_{r}^{16} t_{c}^{18}\right) \\
\left.+q^{4} t_{r}^{14} t_{c}^{18}+q^{6}\left(t_{r}^{20} t_{c}^{18}+t_{r}^{22} t_{c}^{20}\right)+2 q^{8} t_{r}^{20} t_{c}^{20}+q^{10}\left(t_{r}^{18} t_{c}^{20}+t_{r}^{20} t_{c}^{22}\right)\right) \\
+a^{10}\left(q^{-14} t_{r}^{7} t_{c}^{5}+q^{-12}\left(t_{r}^{5} t_{c}^{5}+t_{r}^{7} t_{c}^{7}\right)+q^{-10} t_{r}^{5} t_{c}^{7}+q^{-8} t_{r}^{13} t_{c}^{9}+q^{-6}\left(2 t_{r}^{11} t_{c}^{9}+t_{r}^{13} t_{c}^{11}\right)\right. \\
+q^{-4}\left(t_{r}^{9} t_{c}^{9}+3 t_{r}^{11} t_{c}^{11}\right)+q^{-2}\left(2 t_{r}^{9} t_{c}^{11}+t_{r}^{11} t_{c}^{13}\right)+\left(t_{r}^{9} t_{c}^{13}+t_{r}^{17} t_{r}^{13}\right) \\
+q^{2}\left(2 t_{r}^{15} t_{c}^{13}+t_{r}^{17} t_{c}^{15}\right)+q^{4}\left(t_{r}^{13} t_{c}^{13}+3 t_{r}^{15} t_{c}^{15}\right)+q^{6}\left(2 t_{r}^{13} t_{c}^{15}+t_{r}^{15} t_{c}^{17}\right) \\
\left.+q^{8} t_{r}^{13} t_{c}^{17}+q^{10} t_{r}^{19} t_{c}^{17}+q^{12}\left(t_{r}^{17} t_{c}^{17}+t_{r}^{19} t_{c}^{19}\right)+q^{14} t_{r}^{17} t_{c}^{19}\right) \\
+a^{8}\left(q^{-16}+q^{-10} t_{r}^{6} t_{c}^{4}+q^{-8}\left(t_{r}^{4} t_{c}^{4}+t_{r}^{6} t_{c}^{6}\right)+q^{-6} t_{r}^{4} t_{c}^{6}+q^{-4} t_{r}^{12} t_{c}^{8}+q^{-2} t_{r}^{10} t_{c}^{8}+t_{r}^{8} t_{c}^{8}\right. \\
\left.+t_{r}^{10} t_{c}^{10}+q^{2} t_{r}^{8} t_{c}^{10}+q^{4} t_{r}^{8} t_{c}^{12}+q^{6} t_{r}^{14} t_{c}^{12}+q^{8}\left(t_{r}^{12} t_{c}^{12}+t_{r}^{14} t_{c}^{14}\right)+q^{10} t_{r}^{12} t_{c}^{14}+q^{16} t_{r}^{16} t_{c}^{16}\right) .
\end{array}
$$

Let us list the various differentials and their $\left(a, q, t_{r}, t_{c}\right)$-degrees.

1. Categorification:

$$
\mathcal{P} \boxplus\left(3_{1}\right)\left(a, q, t_{r}=-1, t_{c}=1\right)=P \boxplus\left(3_{1}\right)(a, q)=\mathcal{P} \boxplus\left(3_{1}\right)\left(a, q, t_{r}=1, t_{c}=-1\right) .
$$

2. Canceling differentials: There exist differentials $d_{2 \mid 0}^{\text {col }}$ and $d_{0 \mid 2}^{\text {col }}$, with degrees $(-2,4,-1,-1)$ and $(-2,-4,-5,-5)$, respectively. The remaining generators have degrees $(8,-16,0,0)$ and $(8,16,16,16)$, respectively.

3. Row-removing differentials: There exist differentials $d_{3 \mid 0}^{\text {col }}$ and $d_{1 \mid 2}^{\text {col of }}$ degrees $(-2,6,-3,-1)$ and $(-2,-2,-7,-5)$, respectively, such that

$$
H^{*}\left(\mathcal{H}^{\boxplus}\left(3_{1}\right), d_{3 \mid 0}^{\text {col }}\right) \cong \mathcal{H}^{\square}\left(3_{1}\right), \quad H^{*}\left(\mathcal{H}^{\boxplus}\left(3_{1}\right), d_{1 \mid 2}^{\text {col }}\right) \cong \mathcal{H}^{\square}\left(3_{1}\right) .
$$

4. Column-removing differentials: There exist differentials $d_{0 \mid 3}^{\text {col }}$ and $d_{2 \mid 1}^{\text {col }}$ of degrees $(-2,-6,-5,-7)$ and $(-2,2,-1,-3)$, respectively, such that

$$
H^{*}\left(\mathcal{H}^{\boxplus}\left(3_{1}\right), d_{0 \mid 3}^{\text {col }}\right) \cong \mathcal{H}^{\boxminus}\left(3_{1}\right), \quad H^{*}\left(\mathcal{H}^{\boxplus}\left(3_{1}\right), d_{2 \mid 1}^{\text {col }}\right) \cong \mathcal{H}^{\boxminus}\left(3_{1}\right) .
$$

5. Universal colored differentials: There exist differentials $d^{\uparrow}$ and $d^{\leftarrow}$ of degrees $(0,2,-2,0)$ and $(0,2,0,2)$, respectively, such that

$$
H^{*}\left(\mathcal{H}^{\boxplus}\left(3_{1}\right), d^{\uparrow}\right) \cong \mathcal{H}^{\square}\left(3_{1}\right), \quad H^{*}\left(\mathcal{H}^{\boxplus}\left(3_{1}\right), d^{\leftarrow}\right) \cong \mathcal{H}^{\boxminus}\left(3_{1}\right) .
$$

The remaining properties are best seen in $\left(a, Q, t_{r}, t_{c}\right)$-gradings. Recall that in this case the $Q$-grading can be expressed via the other ones by

$$
Q=\left(q+t_{r}-t_{c}\right) / 2 \text {. }
$$

We denote the $\square$-colored homology in these four gradings by $\widetilde{\mathcal{H}} \boxplus\left(3_{1}\right)$, and the corresponding four-variable Poincaré polynomial by $\widetilde{\mathcal{P}} \boxplus\left(3_{1}\right)\left(a, Q, t_{r}, t_{c}\right)$. In terms of $\mathcal{P} \boxplus\left(3_{1}\right)$, we have

$$
\widetilde{\mathcal{P}} \boxplus\left(3_{1}\right)\left(a, Q, t_{r}, t_{c}\right)=\mathcal{P} \boxplus\left(3_{1}\right)\left(a, Q^{1 / 2}, t_{r} Q^{1 / 2}, t_{c} Q^{-1 / 2}\right) .
$$


6. Self-symmetry:

$$
\widetilde{\mathcal{H}}_{i, j, k, l}^{\boxplus}\left(3_{1}\right) \cong \widetilde{\mathcal{H}}_{i,-j, k-2 j, l-2 j}^{\boxplus}\left(3_{1}\right) .
$$

7. Mirror symmetry: Since $\boxplus^{t}=\boxplus$, the mirror symmetry in this case is another, different symmetry on $\widetilde{\mathcal{H}} \boxplus\left(3_{1}\right)$ :

$$
\widetilde{\mathcal{H}}_{i, j, k, l}^{\boxplus}\left(3_{1}\right) \cong \widetilde{\mathcal{H}}_{i, j, l, k}^{\boxplus}\left(3_{1}\right) .
$$

8. Refined exponential growth:

$$
\widetilde{\mathcal{P}} \boxplus\left(3_{1}\right)\left(a, Q, t_{r}, t_{c}=1\right)=\left(\widetilde{\mathcal{P}}^{\boxminus}\left(3_{1}\right)\left(a, Q, t_{r}, t_{c}=1\right)\right)^{2} .
$$

3.5. $(2,2,2)$-representation. In this section we give the result for the $\boxplus$-colored homology of the trefoil. Although this homology is very large (729-dimensional) it contains all colored differentials predicted in Section 2.5 . Due to its size, and in order to simplify the presentation, we give the homology and the differentials in $\left(a, q, t_{c}\right)$-gradings, and simply denote $t_{c}$ by $t$. In $\left(a, q, t_{c}\right)$-gradings the Poincaré polynomial is given by

$$
\begin{aligned}
& \mathcal{P} \boxplus\left(3_{1}\right)=a^{12}\left(q^{-36}+q^{-30} t^{4}+q^{-28}\left(t^{4}+t^{6}\right)+q^{-26}\left(t^{4}+t^{6}\right)+q^{-24}\left(t^{6}+t^{8}\right)+q^{-22} t^{8}\right. \\
& +q^{-20}\left(2 t^{8}+t^{10}\right)+q^{-18}\left(t^{8}+2 t^{10}\right)+q^{-16}\left(t^{8}+2 t^{10}+t^{12}\right)+q^{-14}\left(t^{10}+2 t^{12}\right) \\
& +q^{-12}\left(3 t^{12}+t^{14}\right)+q^{-10}\left(2 t^{12}+2 t^{14}\right)+q^{-8}\left(t^{12}+3 t^{14}\right)+q^{-6}\left(t^{12}+2 t^{14}+t^{16}\right) \\
& +q^{-4}\left(t^{14}+2 t^{16}\right)+2 q^{-2} t^{16}+q^{0}\left(2 t^{16}+2 t^{18}\right)+q^{2}\left(t^{16}+2 t^{18}\right)+q^{4}\left(t^{16}+2 t^{18}+t^{20}\right) \\
& \left.+q^{6}\left(t^{18}+t^{20}\right)+q^{8} t^{20}+q^{10} t^{20}+q^{12}\left(t^{20}+t^{22}\right)+q^{14}\left(t^{20}+t^{22}\right)+q^{16} t^{22}+q^{24} t^{24}\right) \\
& +a^{14}\left(q^{-36} t^{5}+q^{-34}\left(t^{5}+t^{7}\right)+q^{-32}\left(t^{5}+t^{7}\right)+q^{-30}\left(t^{7}+t^{9}\right)+q^{-28}\left(2 t^{9}+t^{11}\right)\right. \\
& +q^{-26}\left(3 t^{9}+3 t^{11}\right)+q^{-24}\left(2 t^{9}+5 t^{11}+t^{13}\right)+q^{-22}\left(t^{9}+4 t^{11}+3 t^{13}\right) \\
& +q^{-20}\left(2 t^{11}+5 t^{13}+t^{15}\right)+q^{-18}\left(5 t^{13}+4 t^{15}\right)+q^{-16}\left(4 t^{13}+7 t^{15}+t^{17}\right) \\
& +q^{-14}\left(2 t^{13}+8 t^{15}+3 t^{17}\right)+q^{-12}\left(t^{13}+5 t^{15}+6 t^{17}\right)+q^{-10}\left(2 t^{15}+7 t^{17}+2 t^{19}\right) \\
& +q^{-8}\left(6 t^{17}+5 t^{19}\right)+q^{-6}\left(4 t^{17}+8 t^{19}+t^{21}\right)+q^{-4}\left(2 t^{17}+8 t^{19}+3 t^{21}\right) \\
& +q^{-2}\left(t^{17}+5 t^{19}+5 t^{21}\right)+q^{0}\left(2 t^{19}+5 t^{21}+t^{23}\right)+q^{2}\left(4 t^{21}+2 t^{23}\right)+q^{4}\left(3 t^{21}+4 t^{23}\right) \\
& +q^{6}\left(2 t^{21}+5 t^{23}+t^{25}\right)+q^{8}\left(t^{21}+4 t^{23}+2 t^{25}\right)+q^{10}\left(2 t^{23}+2 t^{25}\right)+q^{12} t^{25}+q^{14} t^{25} \\
& \left.+q^{16}\left(t^{25}+t^{27}\right)+q^{18}\left(t^{25}+t^{27}\right)+q^{20} t^{27}\right)+a^{16}\left(q^{-34}\left(t^{10}+t^{12}\right)+q^{-32}\left(t^{10}+2 t^{12}\right)\right. \\
& +q^{-30}\left(t^{10}+3 t^{12}+t^{14}\right)+q^{-28}\left(2 t^{12}+2 t^{14}\right)+q^{-26}\left(t^{12}+3 t^{14}+2 t^{16}\right) \\
& +q^{-24}\left(3 t^{14}+5 t^{16}+t^{18}\right)+q^{-22}\left(2 t^{14}+8 t^{16}+3 t^{18}\right)+q^{-20}\left(t^{14}+7 t^{16}+6 t^{18}\right) \\
& +q^{-18}\left(4 t^{16}+8 t^{18}+2 t^{20}\right)+q^{-16}\left(t^{16}+7 t^{18}+5 t^{20}\right)+q^{-14}\left(5 t^{18}+10 t^{20}+2 t^{22}\right) \\
& +q^{-12}\left(2 t^{18}+11 t^{20}+5 t^{22}\right)+q^{-10}\left(t^{18}+9 t^{20}+9 t^{22}+t^{24}\right)+q^{-8}\left(4 t^{20}+9 t^{22}+2 t^{24}\right) \\
& +q^{-6}\left(t^{20}+7 t^{22}+5 t^{24}\right)+q^{-4}\left(4 t^{22}+7 t^{24}+t^{26}\right)+q^{-2}\left(2 t^{22}+9 t^{24}+3 t^{26}\right) \\
& +q^{0}\left(t^{22}+7 t^{24}+6 t^{26}\right)+q^{2}\left(4 t^{24}+6 t^{26}+t^{28}\right)+q^{4}\left(t^{24}+4 t^{26}+t^{28}\right)+q^{6}\left(2 t^{26}+2 t^{28}\right) \\
& \left.+q^{8}\left(t^{26}+2 t^{28}\right)+q^{10}\left(t^{26}+3 t^{28}+t^{30}\right)+q^{12}\left(2 t^{28}+t^{30}\right)+q^{14}\left(t^{28}+t^{30}\right)\right)
\end{aligned}
$$




$$
\begin{array}{r}
+a^{18}\left(q^{-32} t^{17}+q^{-30}\left(t^{15}+2 t^{17}+t^{19}\right)+q^{-28}\left(3 t^{17}+2 t^{19}\right)+q^{-26}\left(2 t^{17}+3 t^{19}\right)\right. \\
+q^{-24}\left(t^{17}+3 t^{19}+2 t^{21}\right)+q^{-22}\left(2 t^{19}+4 t^{21}+t^{23}\right)+q^{-20}\left(t^{19}+6 t^{21}+4 t^{23}\right) \\
+q^{-18}\left(6 t^{21}+7 t^{23}+t^{25}\right)+q^{-16}\left(3 t^{21}+8 t^{23}+2 t^{25}\right)+q^{-14}\left(t^{21}+6 t^{23}+4 t^{25}\right) \\
+q^{-12}\left(3 t^{23}+6 t^{25}+t^{27}\right)+q^{-10}\left(t^{23}+7 t^{25}+4 t^{27}\right)+q^{-8}\left(6 t^{25}+7 t^{27}+t^{29}\right) \\
+q^{-6}\left(3 t^{25}+8 t^{27}+2 t^{29}\right)+q^{-4}\left(t^{25}+5 t^{27}+3 t^{29}\right)+q^{-2}\left(2 t^{27}+3 t^{29}\right)+q^{0}\left(t^{27}+3 t^{29}+t^{31}\right) \\
\left.+q^{2}\left(3 t^{29}+2 t^{31}\right)+q^{4}\left(2 t^{29}+3 t^{31}\right)+q^{6}\left(t^{29}+2 t^{31}+t^{33}\right)+q^{8} t^{31}\right)+a^{20}\left(q^{-28}\left(t^{22}+t^{24}\right)\right. \\
+q^{-26}\left(t^{22}+2 t^{24}\right)+q^{-24}\left(t^{22}+3 t^{24}+t^{26}\right)+q^{-22}\left(2 t^{24}+t^{26}\right)+q^{-20}\left(t^{24}+2 t^{26}\right) \\
+q^{-18}\left(2 t^{26}+2 t^{28}\right)+q^{-16}\left(2 t^{26}+4 t^{28}+t^{30}\right)+q^{-14}\left(t^{26}+5 t^{28}+2 t^{30}\right)+q^{-12}\left(3 t^{28}+3 t^{30}\right) \\
+q^{-10}\left(t^{28}+2 t^{30}\right)+q^{-8}\left(2 t^{30}+t^{32}\right)+q^{-6}\left(t^{30}+2 t^{32}\right)+q^{-4}\left(t^{30}+3 t^{32}+t^{34}\right) \\
\left.+q^{-2}\left(2 t^{32}+t^{34}\right)+q^{0}\left(t^{32}+t^{34}\right)\right)+a^{22}\left(q^{-24} t^{29}+q^{-22}\left(t^{29}+t^{31}\right)+q^{-20}\left(t^{29}+t^{31}\right)\right. \\
\left.+q^{-18} t^{31}+q^{-14} t^{33}+q^{-12}\left(t^{33}+t^{35}\right) q^{-10}\left(t^{33}+t^{35}\right)+q^{-8} t^{35}\right) a^{24} q^{-18} t^{36} .
\end{array}
$$

We note that the above Poincaré polynomial, after an appropriate change of variables, matches the result from [14]. In addition, on such a big homology $\mathcal{H}^{\boxplus}\left(3_{1}\right)$ categorifying the reduced $\boxplus$-colored HOMFLYPT polynomial of the trefoil knot, there exist all of the predicted colored differentials. These straightforward, and clearly long and tedious checks are summarized below:

1. Canceling differential $d_{3 \mid 0}^{\text {col }}$ of $\left(a, q, t_{c}\right)$-degree $(-2,6,-1)$ such that the homology $H^{*}\left(\mathcal{H} \boxplus\left(3_{1}\right), d_{3 \mid 0}^{\text {col }}\right)$ is one-dimensional, and the remaining generator has $\left(a, q, t_{c}\right)$-degree $(12,-36,0)$.

2. Canceling differential $d_{0 \mid 2}^{\text {col }}$ of degree $(-2,-4,-5)$ such that the homology $H^{*}\left(\mathcal{H}^{\boxplus}\left(3_{1}\right), d_{0 \mid 2}^{\text {col }}\right)$ is one-dimensional, and the remaining generator has $\left(a, q, t_{c}\right)$-degree $(12,24,24)$.

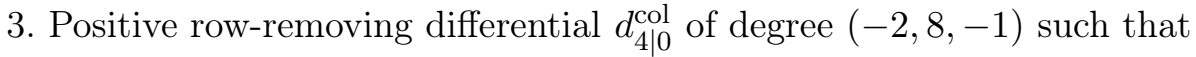

$$
H^{*}\left(\mathcal{H}^{\boxplus}\left(3_{1}\right), d_{4 \mid 0}^{\text {col }}\right) \cong \mathcal{H}^{\square}\left(3_{1}\right) \text {. }
$$

4. Negative row-removing differential $d_{1 \mid 2}^{\text {col }}$ of degree $(-2,-2,-5)$ such that

$$
H^{*}\left(\mathcal{H}^{\boxplus}\left(3_{1}\right), d_{1 \mid 2}^{\text {col }}\right) \cong \mathcal{H}^{\square}\left(3_{1}\right)
$$

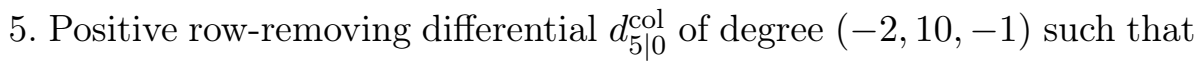

$$
H^{*}\left(\mathcal{H} \boxplus\left(3_{1}\right), d_{5 \mid 0}^{\text {col }}\right) \cong \mathcal{H}^{\boxplus}\left(3_{1}\right)
$$

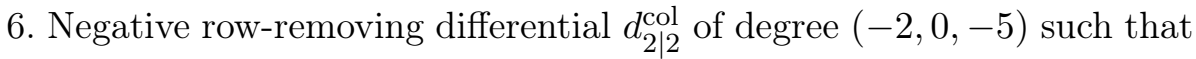

$$
H^{*}\left(\mathcal{H}^{\boxplus}\left(3_{1}\right), d_{2 \mid 2}^{\text {col }}\right) \cong \mathcal{H}^{\boxplus}\left(3_{1}\right)
$$


7. Negative column-removing differential $d_{0 \mid 3}^{\text {col }}$ of degree $(-2,-6,-7)$ such that

$$
H^{*}\left(\mathcal{H}^{\boxplus}\left(3_{1}\right), d_{0 \mid 3}^{\text {col }}\right) \cong \mathcal{H}^{\text {日 }}\left(3_{1}\right) .
$$
that

8. Positive column-removing differential $d_{3 \mid 1}^{\text {col }}$ of degree $(-2,-4,-3)$ such

$$
H^{*}\left(\mathcal{H}^{\boxplus}\left(3_{1}\right), d_{3 \mid 1}^{\text {col }}\right) \cong \mathcal{H}^{⿴ 囗}\left(3_{1}\right) .
$$

9. Universal colored differential $d^{\leftarrow}$ of degree $(0,2,2)$ such that

$$
H^{*}\left(\mathcal{H}^{\boxplus}\left(3_{1}\right), d^{\leftarrow}\right) \cong \mathcal{H}^{\boxminus}\left(3_{1}\right) .
$$

\section{Bosonic and fermionic generators}

4.1. The algebra of BPS states and knot cobordisms. While most of this paper is probably aimed at a more mathematical reader, here we offer a geometric interpretation of some of our observations in the framework more familiar to physicists. Therefore, the purpose of this section is twofold. First, it will help physicists to better understand some of our observations (which, we believe, may go beyond the scope of the present paper and are worth studying further in the context of BPS state counting). Second, the physical interpretation/motivation provides further evidence for the structures of knot homologies discussed in this paper. In Section 6.4 we also comment on the connection with the equivariant instanton counting in the presence of a surface operator.

We begin with a lightning review of the physical interpretation of knot homologies, in which $\mathcal{H}_{\text {knot }}$ is identified with a space of supersymmetric particles (the so-called BPS states) [43, 41, 96]:

$$
\mathcal{H}_{\text {knot }}=\mathcal{H}_{\text {BPS }} \text {. }
$$

In particular, it will allow us to see bosonic and fermionic generators of homological knot invariants and will also help us in Section 6.4 to understand the limit $a \rightarrow 0$. As we shall see, the latter allows one to focus on the "bottom row" of the superpolynomial which turns out to have a very elegant and simple geometric interpretation in the physical framework.

In one of the duality frames, the physical system involves a compactification on a very particular (noncompact) Calabi-Yau 3-fold $X$ in the presence of five-branes supported on a Lagrangian submanifold $L_{K} \subset X$ :

$$
\begin{array}{ll}
\text { space-time: } & \mathbb{R} \times X \times M_{4}, \\
\text { M5-branes: } & \mathbb{R} \times L_{K} \times D .
\end{array}
$$

Specifically, $X$ is the total space of the $\mathcal{O}(-1) \oplus \mathcal{O}(-1)$ bundle over $\mathbb{C} \mathbf{P}^{1}$, while the Lagrangian submanifold $L_{K}$ is determined by a knot $K \subset \mathbf{S}^{3}$ 
(cf. [73, 89, 62]). Furthermore, the Kähler modulus of $X$ is related to the variable $a$ in the superpolynomial:

$$
a=\exp \left(-\operatorname{Vol}\left(\mathbb{C} \mathbf{P}^{1}\right)\right),
$$

whereas the number of five-branes in 4.2 is equal to the number of columns in $|\lambda|$ (see e.g. [73, 63] for further details on how $\lambda$ is encoded in the five-brane configuration (4.2)).

This summary was very telegraphic, of course, but the reader can always consult e.g. 44] for further details, references, and an outline of the relation between different ways of looking at the physical system (4.2). As reviewed there, the system (4.2) was studied in physics as well as in math literature from many different angles, e.g. from the target space point of view [43, 45] that leads to a description of (4.1) in terms of enumerative invariants of $\left(X, L_{K}\right)$, or from the viewpoint of the five-brane [96], or from the viewpoint of the $\mathcal{N}=2$ gauge theory on a 4-manifold $M_{4}$ with ramification [19]. However, only recently [31, 29] has $(4.2)$ been considered from the viewpoint of the two-dimensional theory on $D$, which will be yet another motivation for the discussion in Section 6.4.

Now we are ready to discuss the BPS states that contribute to the reduced and unreduced superpolynomials, $\mathcal{P}^{\lambda}(a, q, t)$ and $\overline{\mathcal{P}}^{\lambda}(a, q, t)$, respectively.

As discussed e.g. in [73, 43, 68, there are two kinds of BPS particles that contribute to (4.1) in the problem at hand: M2-branes supported on bordered surfaces embedded in $X$ with boundary on $L_{K}$, and momentum modes (=D0-branes). The charge of the former is precisely the $a$-grading, while the charge of the latter is the $q$-grading. Both types of states can mix together (forming stable BPS particles that carry both charges) and both can carry a nontrivial spin $j_{3}$, which becomes the third $t$-grading on (4.1). To summarize, the dictionary between the three gradings on the colored HOMFLYPT homology and the charges of the BPS particles is as follows:

$$
\begin{array}{lll}
\text { " } a \text {-grading" }=\beta \in H_{2}\left(X, L_{K}\right) \cong \mathbb{Z} & & \text { ("winding number"), } \\
\text { " } q \text {-grading" }=n \in \mathbb{Z} & & \text { ("D0-brane charge"), } \\
\text { " } t \text {-grading" }=2 j_{3} \in \mathbb{Z} & & \text { (spin) } .
\end{array}
$$

Using this dictionary, we can identify every generator of (4.1) with the corresponding BPS particle. For example, if we see a term $a^{m} q^{n} t^{k}$ in the superpolynomial, the proper translation to the physics language is

$$
\begin{array}{r}
\text { monomial } \\
a^{m} q^{n} t^{k} \subset \mathcal{P}^{\lambda}
\end{array} \leftrightarrow \begin{gathered}
\text { "a BPS particle with winding number } m, \\
D 0 \text {-brane charge } n, \text { and spin } k / 2 " .
\end{gathered}
$$

The particles that carry no winding number $(m=0)$ are usually called simply D0-branes. 
One well-known property of the BPS states in the present setup is that particles that carry nontrivial winding number $m=1$ are fermions, whereas D0-brane states (with $m=0$ ) are bosonic [18, 53. When translated to the language of knot homologies, this statement says that the statistics of the generators in the colored HOMFLYPT homology is correlated to the $a$-grading!

Namely, the generators with trivial $a$-grading are bosonic, whereas the generators with $a$-grading equal to 1 are fermions. This will play a key role in what follows.

Another useful lesson that we can learn from the connection with enumerative (BPS) invariants 4.1)-4.2) is the behavior of colored HOMFLYPT homology with respect to cobordisms. First, however, it is convenient to consider a dual brane system related to 4.2 by a geometric transition $\left({ }^{8}\right)$ [34, 73]:

$$
\begin{aligned}
\text { space-time: } & \mathbb{R} \times T^{*} W \times M_{4}, \\
N \text { M5-branes: } & \mathbb{R} \times W \times D, \\
|\lambda| \text { M5-branes: } & \mathbb{R} \times L_{K} \times D,
\end{aligned}
$$

where $W=S^{3}$ in most of our applications (or its close cousin $W=\mathbb{R}^{3}$ ). This system is relevant to $\operatorname{sl}(N)$-homological knot invariants and has been studied from various points of view in [2, 52, 96]. One advantage of the duality frame (4.6) that will serve us well in building knot cobordisms is that the Lagrangian submanifold $L_{K} \subset T^{*} W$ can be defined simply as the conormal bundle to the knot $K \subset W$.

Now, it is easy to describe how knot cobordisms can be implemented in the physical setup. Since the factor of $\mathbb{R}$ in $(4.2)$ and $(4.6)$ plays the role of the "time" direction, one can replace $\mathbb{R} \times S^{3}$ by a general 4-manifold cobordism $V$ and replace $\mathbb{R} \times K$ embedded in it by a more general knot cobordism $\Sigma \subset V$, as in Figure 10 .

$$
\begin{aligned}
\text { space-time: } & X_{7} \times M_{4}, \\
N \text { M5-branes: } & V \times D, \\
|\lambda| \text { M5-branes: } & L_{\Sigma} \times D .
\end{aligned}
$$

Supersymmetry imposes certain constraints, which ensure that the physical setup here continues to maintain the relation between supersymmetric configurations (BPS states) and functors on knot homologies. Specifically, the 7-manifold $X_{7}$ must be a special holonomy manifold with holonomy group $G_{2}$, while the 4-manifold $L_{\Sigma}$ must be a coassociative submanifold

$\left({ }^{8}\right)$ Sometimes also called "large $N$ duality", which is somewhat of a misnomer since the duality holds for all values of $N$, as long as one is careful with differentials and wall crossing [20, 44]. 
in $X_{7}$. Both can be constructed in a fairly simple way, starting with the data of $V$ and $\Sigma$, respectively.

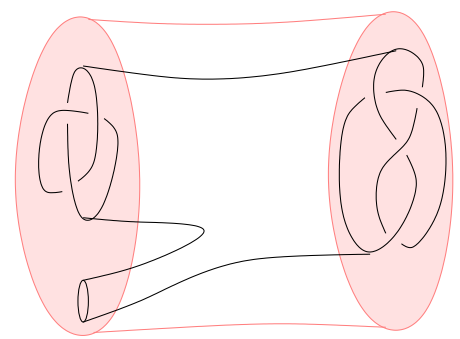

Fig. 10. A real isotropic knot cobordism $\Sigma \subset \mathbb{R} \times S^{3}$ defines a coassociative 4-manifold $L_{\Sigma} \subset \mathbb{R} \times T^{*} S^{3}$.

Just like $T^{*} W$ is a "canonical" Calabi-Yau 3-fold associated to a 3manifold $W$, from a 4-manifold $V$ one can build a "canonical" $G_{2}$-holonomy manifold $X_{7}=\Lambda^{2,+}(V)$ as the total space of the bundle of self-dual 2-forms. This 7-manifold $X_{7}=\Lambda^{2,+}(V)$ replaces $\mathbb{R} \times T^{*} W$ and reduces to it when $V=\mathbb{R} \times W$. In what follows, we restrict our attention only to this case, and moreover assume $W=S^{3}$. Then, given a knot cobordism $\Sigma \subset V=\mathbb{R} \times S^{3}$, we wish to construct a coassociative 4-manifold $L_{\Sigma} \subset \Lambda^{2,+}(V)$ with the property $L_{\Sigma} \cap V=\Sigma$, which is a suitable $G_{2}$-manifold version of the property $L_{K} \cap S^{3}=K$ in (4.6). By analogy with the conormal bundle construction of $L_{K}$, the desired coassociative 4-manifold $L_{\Sigma}$ can be defined as the subbundle of $\Lambda^{2,+}(V)$ over $\Sigma \hookrightarrow V$ such that restriction of the fiber to $\Sigma$ is trivial, provided that $\Sigma$ is a real isotropic minimal surface [51, 54]. Details of this construction and its implications for functors on colored knot homologies will be discussed elsewhere. Here, we simply point out that the existence of such a geometric construction implies the existence of a map

$$
\overline{\mathcal{H}}^{\lambda}(\bigcirc) \otimes \overline{\mathcal{H}}^{\lambda}(K) \rightarrow \overline{\mathcal{H}}^{\lambda}(K) \text {. }
$$

Indeed, applying geometric transition one more time to the setup 4.7) with $V=\mathbb{R} \times S^{3}$ we get a "cobordism version" of 4.2 ):

space-time: $\quad \mathbb{R} \times X \times M_{4}$,

M5-branes: $L_{\Sigma} \times D$,

where $L_{\Sigma}$ is a coassociative 4 -manifold in $\mathbb{R} \times X$. When $\Sigma$ is a link cobordism from $K \sqcup \bigcirc$ to $K$, the setup (4.9) defines a functor on the space of refined open BPS states and, via 4.1), leads to a functor (4.8) on colored HOMFLYPT homology.

Note, that (4.8) is very similar - and, in fact, possibly related - to the conjecture 44] that $\overline{\mathcal{H}}^{\lambda}(K)=\mathcal{H}_{\mathrm{BPS}}^{\text {open }}$ is a module over the algebra of closed 
BPS states $\mathcal{H}_{\mathrm{BPS}}^{\text {closed: }}$

$$
\begin{array}{cc}
\text { refined open BPS states: } & \mathcal{H}_{\mathrm{BPS}}^{\text {open }} \\
\text { refined closed BPS states: } & \mathcal{H}_{\mathrm{BPS}}^{\text {closed }}
\end{array}
$$

Even though we did not pursue the detailed comparison of $\mathcal{A}=\overline{\mathcal{H}}(\bigcirc)$ and the algebra $\mathcal{H}_{\mathrm{BPS}}^{\text {closed }}$ here, it is worth mentioning that the two have some similarities. For instance, much like $\overline{\mathcal{H}}(\bigcirc)$ has $\operatorname{sl}(N)$-specializations indexed by $N$ and realized by the action of differentials $d_{N}$ (see below), the space $\mathcal{H}_{\mathrm{BPS}}^{\text {closed }}$ for the problem at hand also has an infinite set of chambers, conveniently labeled by $N$ (see Figure 11). Moreover, in the $N$ th chamber the space $\mathcal{H}_{\mathrm{BPS}}^{\text {closed }}$ has $N$ stable BPS objects [68]: D0/D2 bound states of charge $(n, \beta)=(j, 1)$, where $0 \leq j<N$, nicely matching the fact that $\overline{\mathcal{H}}^{\operatorname{sl}(N), \square}(\bigcirc)$ is also $N$-dimensional.

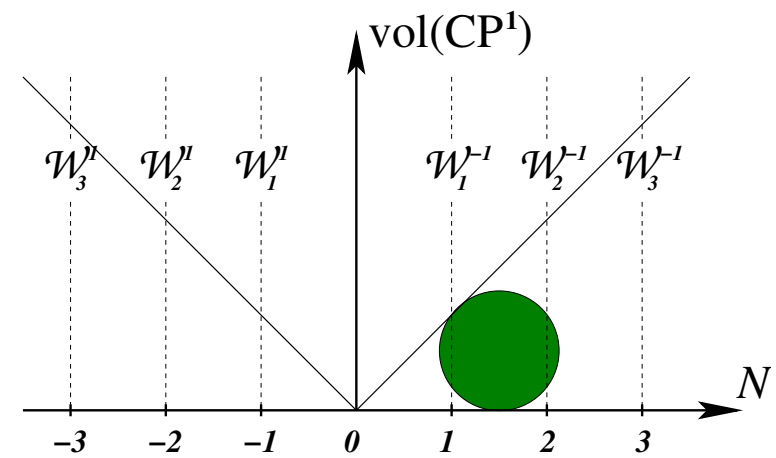

Fig. 11. Walls and chambers for the conifold $X$ in 4.2

4.1.1. Supersymmetry and $\mathrm{gl}(n \mid m)$ representations. One of the structures we predict on HOMFLYPT homology is the structure of $\mathrm{gl}(\mathrm{n} \mid \mathrm{m})$ differentials - see Section 2.4, and in general, $\mathrm{gl}(n \mid m)$ representations. This structure has appeared in the physics literature [91, 95] as a symmetry of brane/antibrane systems, which makes it especially easy to implement in the brane system 4.2 or, better yet, in the dual system 4.6 where the rank $N$ enters as the number of five-branes. In this framework, replacing $\operatorname{sl}(N)$ by a Lie superalgebra $g l(n \mid m)$ can be easily implemented by replacing $N$ five-branes with a system of $n$ five-branes and $m$ antibranes.

Another, more supersymmetric way to realize quantum and homological invariants of knots labeled by $\operatorname{gl}(n \mid m)$ representations is to consider $n$ fivebranes supported on $\mathbb{R} \times W \times D$ exactly as in (4.6) and introduce $m$ new fivebranes supported on $\mathbb{R} \times W \times D^{\prime}$, such that $\mathbb{C} \cong D^{\prime} \subset M_{4}$ and $D \cap D^{\prime}=\{0\}$ : 
space-time: $\mathbb{R} \times T^{*} W \times M_{4}$,

$n$ M5-branes: $\mathbb{R} \times W \times D$,

$m$ M5-branes: $\mathbb{R} \times W \times D^{\prime}$.

For instance, if $M_{4} \cong \mathbb{C}^{2}$ is parametrized by coordinates $\left(z_{1}, z_{2}\right)$, then we can choose $D=\left\{z_{1}=0\right\}$ and $D^{\prime}=\left\{z_{2}=0\right\}$. Furthermore, identifying $M_{4}$ with the Taub-NUT space and reducing on the circle fiber takes us from M-theory to type IIA string theory with a system of D4-branes and D6-branes shown in Figure 12 .

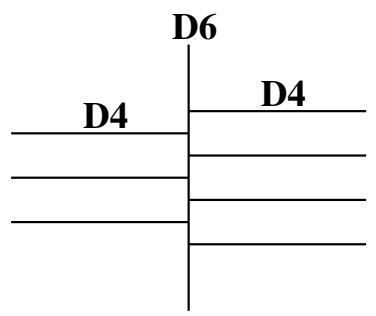

Fig. 12. In the approach of [96], homological invariants of knots labeled by representations of $\operatorname{gl}(n \mid m)$ can be realized by a system of $n$ semiinfinite D4-branes ending on one side of the D6-brane, and $m$ semiinfinite D4-branes ending on the other side of the D6-brane.

Then, as in [96], homological gl $(n \mid m)$ knot invariants can be obtained by "counting" solutions to the following partial differential equations on a 5-manifold $\mathbb{R} \times W \times \mathbb{R}_{y}$ :

$$
F^{+}-\frac{1}{4} B \times B-\frac{1}{2} D_{y} B=0, \quad F_{y i}+D^{j} B_{j i}=0
$$

with the prescribed behavior near the "interface" $y=0$ :

$$
B \simeq B_{0}^{( \pm)} / y \quad \text { as } y \rightarrow \pm 0 .
$$

Here, $B$ is a section of $\Omega^{2,+}(\mathbb{R} \times W) \otimes \operatorname{ad}(E)$, so that

$$
(B \times B)_{i j}=\sum_{k}\left[B_{i k}, B_{j k}\right] .
$$

We relegate further study of this physical system to a future work.

4.2. Colored polynomials of the unknot. For a Young diagram $\lambda$ and a box $x$ in it we define the arm $a(x)$, leg $l(x)$, co-arm $a^{\prime}(x)$ and coleg $l^{\prime}(x)$ as shown in Figure 13 . The hook-length of $x$ is defined as $h(x)=$ $a(x)+l(x)+1$, and the content of $x$ is defined as $c(x)=a^{\prime}(x)-l^{\prime}(x)$.

Let $\lambda$ be a Young diagram with $k$ boxes, let $V_{\lambda}$ denote the irreducible representation of $S_{k}$ labeled by $\lambda$, and let $U_{\lambda}=\mathbb{S}_{\lambda}\left(\mathbb{C}^{N}\right)$ denote the irreducible representation of $\operatorname{sl}(N)$ labeled by $\lambda$. The $\operatorname{sl}(N)$ quantum invariant of the $\lambda$ colored unknot coincides with the character of the representation $U_{\lambda}$ and is 


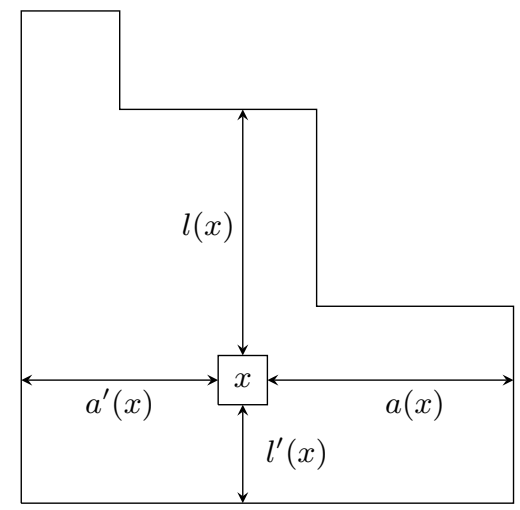

Fig. 13. Arm, leg, co-arm and co-leg

given by 4.12 below. This character is given by the hook formula

$$
\operatorname{ch} U_{\lambda}=\prod_{x \in \lambda} \frac{1-\mathbf{q}^{N+c(x)}}{1-\mathbf{q}^{h(x)}} .
$$

If we substitute $\mathbf{q}^{N}=A$, we get

$$
P^{\lambda}(A, \mathbf{q})=\prod_{x \in \lambda} \frac{1-A \mathbf{q}^{c(x)}}{1-\mathbf{q}^{h(x)}} .
$$

Let $M_{\lambda}\left(\mathbf{q}, \mathbf{t} ; p_{i}\right)$ be a Macdonald polynomial corresponding to a diagram $\lambda$, where $p_{i}$ denotes the $i$ th power sum. Recall that its evaluation can be computed by the formula (see [65])

$$
M_{\lambda}^{*}(A, \mathbf{q}, \mathbf{t})=M_{\lambda}\left(p_{i}=\frac{1-A^{i}}{1-\mathbf{t}^{i}}\right)=\prod_{x \in \lambda} \frac{\mathbf{t}^{l^{\prime}(x)}-A \mathbf{q}^{a^{\prime}(x)}}{1-\mathbf{q}^{a(x)} \mathbf{t}^{l(x)+1}} .
$$

Note that at $\mathbf{q}=\mathbf{t}$ Macdonald polynomials degenerate to Schur polynomials and (4.13) degenerates to 4.12). In all approaches based on the refined BPS invariants the (unreduced) colored superpolynomials of the unknot are given by the Macdonald dimensions

$$
\overline{\mathcal{P}}^{\lambda}(\bigcirc)=M_{\lambda}^{*}(A, \mathbf{q}, \mathbf{t}) .
$$

In order to write these expressions with positive coefficients, one needs to convert to the variables $(a, q, t)$ that we use throughout the paper:

$$
A=-a^{2} t, \quad \mathbf{q}=q^{2} t^{2}, \quad \mathbf{t}=q .
$$

The first few values of (4.14 read

$$
\overline{\mathcal{P}}^{\square}(\bigcirc)=\frac{1-A^{2}}{1-\mathbf{t}^{2}}=\frac{1+a^{2} t}{1-q^{2}},
$$




$$
\overline{\mathcal{P}}^{\square}(\bigcirc)=\frac{\left(1-A^{2}\right)\left(1-A^{2} \mathbf{q}^{2}\right)}{\left(1-\mathbf{t}^{2}\right)\left(1-\mathbf{q}^{2} \mathbf{t}^{2}\right)}=\frac{\left(1+a^{2} t\right)\left(1+a^{2} q^{2} t^{3}\right)}{\left(1-q^{2}\right)\left(1-q^{4} t^{2}\right)},
$$

where we normalized by overall powers of $A$, $\mathbf{q}$, and $\mathbf{t}$, so that all expressions (4.14) have the form of a power series $\overline{\mathcal{P}}^{\lambda}(\bigcirc)=1+\cdots$.

The variable $t$ will be also denoted by $t_{c}$. The equation (4.14) in these variables has the form

$$
\overline{\mathcal{P}}^{\lambda}(\bigcirc)=\prod_{x \in \lambda} \frac{1+a^{2} q^{2 c(x)} t^{2 a^{\prime}(x)+1}}{1-q^{2 h(x)} t^{2 a(x)}} .
$$

Note that the right-hand side is a polynomial in $a$ and a series in $q$ with all nonnegative coefficients.

4.3. Schur-Weyl duality. Following [58], one can identify the uncolored HOMFLYPT homology of the unknot with the polynomial algebra in one even and one odd variable, which is isomorphic to the space of differential forms on a line. More precisely, one can write

$$
\mathcal{H}^{\square}(\bigcirc)=\Omega^{\bullet}(\mathbb{C})=\mathbb{C}[x, d x] .
$$

The $\operatorname{sl}(N)$ differential is given by the Koszul complex: $d_{N}(x)=0$ and $d_{N}(d x)=x^{N}$. It is clear that $H^{*}\left(\mathcal{H}^{\square}, d_{N}\right) \simeq U_{\square}(\operatorname{sl}(N))$. The $k$ th tensor power of this construction will give

$$
\mathcal{H}^{\square^{\otimes k}}=\Omega^{\bullet}\left(\mathbb{C}^{k}\right)=\mathbb{C}\left[x_{1}, \ldots, x_{k} ; d x_{1}, \ldots, d x_{k}\right],
$$

and the differentials are given by $d_{N}\left(x_{i}\right)=x_{i}^{N}$. Following the ideas of [45], define

$$
\mathcal{H}_{\text {alg }}^{\lambda}:=\operatorname{Hom}_{S_{k}}\left(V_{\lambda}, \Omega^{\bullet}\left(\mathbb{C}^{k}\right)\right) .
$$

It is naturally equipped with two gradings: the $a$-grading is given by the degree of a differential form, and the $q$-grading is a standard polynomial grading. In other words, the $(a, q)$-degree for $x_{i}$ equals $q^{2 i}$, and for $d x_{i}$ it equals $a^{2}$.

Proposition 4.1. For any $\lambda$ the $(a, q)$-character of the space $\mathcal{H}_{\text {alg }}^{\lambda}$ agrees with the colored HOMFLYPT polynomial of the unknot.

Proof. It follows from the results of [59] (see also [67]) that the character of $\mathcal{H}^{\lambda}$ equals

$$
\operatorname{ch} \mathcal{H}_{\mathrm{alg}}^{\lambda}(a, q)=\prod_{(i, j) \in \lambda} \frac{q^{2(i-1)}+a^{2} q^{2(j-1)}}{1-q^{2 h(i, j)}} .
$$

Therefore, by 4.12,

$$
\operatorname{ch} \mathcal{H}_{\mathrm{alg}}^{\lambda}(a, q)=q^{\nu(\lambda)} P^{\lambda}(a \sqrt{-1}, q),
$$

where $\nu(\lambda)=\sum(i-1) \lambda_{i}$. 
For the symmetric representation we have

$$
\mathcal{H}_{\text {alg }}^{S^{k}}=\left(\Omega^{\bullet}\left(\mathbb{C}^{k}\right)\right)^{S_{k}}=\mathbb{C}\left[u_{1}, \ldots, u_{k} ; d u_{1}, \ldots, d u_{k}\right],
$$

where the $u_{i}$ denote the elementary symmetric polynomials. Note that the $(a, q)$-degree for $u_{i}$ equals $q^{2 i}$, and for $d u_{i}=\sum \frac{\partial u_{i}}{\partial x_{j}} d x_{j}$ it equals $a^{2} q^{2 i-2}$. Therefore the bigraded Poincaré series of $\mathcal{H}_{\text {alg }}^{S^{k}}$ has the form

$$
P\left(\mathcal{H}^{S^{k}}\right)=\prod_{i=1}^{k} \frac{1+a^{2} q^{2 i-2}}{1-q^{2 i}},
$$

in agreement with 4.12) (keeping in mind the change of variables 4.15), and (4.21).

Conjecture 4.2. There exists an additional t-grading on the space $\mathcal{H}_{\text {alg }}^{\lambda}$ such that the $(a, q, t)$-graded Poincaré series agrees with (4.18).

For example, for $\lambda=S^{k}$ the space $\mathcal{H}_{\text {alg }}^{S^{k}}$ is a free polynomial algebra in even and odd generators and one can easily assign an additional $t$-grading to its generators.

4.4. Colored homology of the unknot. Consider a free supercommutative algebra $\mathcal{A}$ with even generators $u_{1}, \ldots, u_{r}$ and odd generators $\xi_{1}, \ldots, \xi_{s}$. Suppose that the algebra is graded so that $\operatorname{deg} u_{i}=\alpha_{i}, \operatorname{deg} \xi_{i}=\beta_{i}$. It is well known that the Hilbert series of this algebra is given by

$$
H_{\alpha, \beta}(q)=\frac{\prod_{i=1}^{s}\left(1+q^{\beta_{i}}\right)}{\prod_{i=1}^{r}\left(1-q^{\alpha_{i}}\right)} .
$$

The similarity of this formula to 4.12 and 4.18 suggests the following construction. We conjecture that the homology $\mathcal{H}^{\lambda}$ of the unknot colored by a rectangle with $R$ rows and $C$ columns is isomorphic to the free graded supercommutative algebra $\mathcal{A}$.

As we will mostly discuss rectangle-shaped Young diagrams, let us fix some notations for them. Let $\lambda$ be a rectangle with $R$ rows and $C$ columns. For a box $x=(i, j), 1 \leq i \leq C, 1 \leq j \leq R$, in it we have

$$
\begin{aligned}
a(x) & =C-i, \quad l(x)=R-j, \quad h(x)=R+C-i-j+1, \\
a^{\prime}(x) & =i-1, \quad l^{\prime}(x)=j-1 .
\end{aligned}
$$

CONJECTURE 4.3. The HOMFLYPT homology of the unknot colored by the $R \times C$ rectangle $\lambda$ is a free polynomial algebra with one bosonic generator $u_{i j}$ and one fermionic generator $\xi_{i j}$ per box $(i, j) \in \lambda$. Their gradings are given by

$$
\begin{aligned}
\left(q, a, t_{c}\right)\left[u_{i j}\right] & =(2(R+C-i-j+1), 0,2(C-i)), \\
\left(q, a, t_{c}\right)\left[\xi_{i j}\right] & =(2(i-j), 2,2 i-1) .
\end{aligned}
$$


We define the fourth grading using the mirror symmetry between $R \times C$ and $C \times R$ rectangles. To write down the isomorphism $M_{\lambda}: \mathcal{H}^{\lambda} \rightarrow \mathcal{H}^{\lambda^{t}}$, one can define it on the level of generators. We let

$$
M_{\lambda}\left(u_{i j}\right):=u_{R-j+1, C-i+1}, \quad M_{\lambda}\left(\xi_{i j}\right):=\xi_{j i} .
$$

The correspondence between boxes is shown in Figure 14. Note that for bosonic generators we fix the southeast corner of the rectangle (marked with $*$ ) while for the fermionic ones we fix the southwest corner. We define the grading $t_{r}$ using the mirror symmetry relation $t_{r}(w)=t_{c}\left(M_{\lambda}(w)\right)$, so

$$
t_{r}\left(u_{i j}\right)=2 j-2, \quad t_{r}\left(\xi_{i j}\right)=2 j-1 .
$$

From the formula

$$
Q=\frac{q+t_{r}-t_{c}}{R}
$$

we get

$$
Q\left(u_{i j}\right)=2, \quad Q\left(\xi_{i j}\right)=0 .
$$

For $\lambda=S^{r}$, Conjecture 4.3 was proven by Hogancamp:
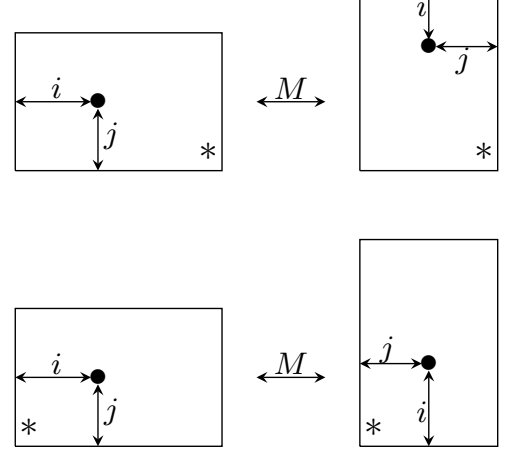

Fig. 14. Mirror symmetry for $u_{i j}$ (upper pair) and for $\xi_{i j}$ (lower pair)

THEOREM 4.4 ([50]). The $S^{r}$-colored HOMFLYPT homology of the un$k n o t$ is isomorphic to the free polynomial algebra in $r$ even and $r$ odd generators.

4.5. Potentials and differentials: antisymmetric case. Let $\mathcal{H}^{\lambda}(K)$ and $\mathcal{H}^{\mathrm{sl}(N), \lambda}(K)$ respectively denote the HOMFLYPT and $\operatorname{sl}(N)$ homology of a knot $K$. By Theorem 1.1 there exists a differential $d_{N}$ of $(a, q)$-degree $(-2,2 N)$ such that

$$
\mathcal{H}^{\mathrm{sl}(N), \lambda}(K)=H^{*}\left(\mathcal{H}^{\lambda}(K), d_{N}\right) .
$$

Note that $d_{N}$ preserves the grading $N a+q$, and its Euler characteristic with respect to this grading equals $P^{\lambda}\left(K ; a=q^{N}, q\right)=P^{\mathrm{sl}(N), \lambda}(K)$. 
On the other hand, it is known [45] that for some diagrams $\lambda$ one can construct a family of potentials $W_{\lambda, N}\left(u_{1}, \ldots, u_{d}\right)$ such that $\overline{\mathcal{H}}^{\mathrm{sl}(N), \lambda}(\bigcirc)$ coincides with the Jacobi ring $J=\mathbb{C}\left[u_{i}\right] /\left(\frac{\partial W_{\lambda, N}}{\partial u_{i}}\right)$. More generally, $\overline{\mathcal{H}}^{\mathrm{sl}(N), \lambda}(\bigcirc)$ is expected to coincide with the Hochschild homology of the category of matrix factorizations of $W_{\lambda, N}$, that is, with the homology of the Koszul complex associated with the sequence of the partial derivatives of $W_{\lambda, N}$. The latter homology contains the Jacobi ring $J$, and is equal to it if and only if $W_{\lambda, N}$ has only isolated singularities. In other words, if the potentials $W_{\lambda, N}\left(u_{1}, \ldots, u_{d}\right)$ exist then one can consider the free supercommutative algebra $\mathcal{A}$ with even generators $u_{1}, \ldots, u_{d}$, odd generators $\xi_{1}, \ldots, \xi_{d}$ and the differential

$$
d_{N}: \mathbb{C}\left[u_{i}, \xi_{j}\right] \rightarrow \mathbb{C}\left[u_{i}, \xi_{j}\right], \quad d_{N}\left(\xi_{i}\right)=\frac{\partial W_{\lambda, N}}{\partial u_{i}}, \quad d_{N}\left(u_{i}\right)=0 .
$$

This matches the above construction of differentials if one identifies $u_{i}$ and $\xi_{i}$ with the even and odd generators of $\overline{\mathcal{H}}^{\lambda}(\bigcirc)$, so that $\mathbb{C}\left[u_{i}, \xi_{j}\right]=\overline{\mathcal{H}}^{\lambda}(\bigcirc)$.

The potential $W_{\Lambda^{k}, N}$ was found in [45]:

$$
W_{\Lambda^{k}, N}\left(u_{1}, \ldots, u_{k}\right)=\operatorname{Coef}_{N+1}\left[\ln \left(1+z u_{1}+\cdots+z^{k} u_{k}\right)\right],
$$

where Coef $_{k}$ denotes the coefficient of $z^{k}$. This potential has an isolated singularity at the origin, and the Jacobi rings of $W_{\Lambda^{k}, N}$ and $W_{\Lambda^{N-k, N}}$ are isomorphic.

Proposition 4.5. Up to some change of variables $\left(u_{i}\right) \mapsto\left(v_{i}\right)$ we have

$$
\begin{aligned}
W_{\Lambda^{k}, 2 k-j}\left(u_{1}, \ldots, u_{k}\right)= & W_{\Lambda^{k-j}, 2 k-j}\left(v_{1}, \ldots, v_{k-j}\right) \\
& +\frac{1}{2} \sum_{s=1}^{j} v_{k-j+s} v_{k+1-s} .
\end{aligned}
$$

Proof. Let us explain the construction of $v_{i}$ in general. Consider supplementary variables $w_{j}$ defined by

$$
w_{s}=\operatorname{Coef}_{j}\left[\frac{1+z u_{1}+\cdots+z^{k} u_{k}}{1+z u_{1}+\cdots+z^{k-j} u_{k-j}}\right] .
$$

Clearly, $w_{s}=0$ for $s \leq k-j$, and the change of variables

$$
\left(u_{1}, \ldots, u_{k}\right) \mapsto\left(u_{1}, \ldots, u_{k-j}, w_{k-j+1}, \ldots, w_{k}\right)
$$

is invertible. Now

$$
\begin{aligned}
W_{\Lambda^{k}, 2 k-j}\left(u_{1}, \ldots, u_{k}\right)- & W_{\Lambda^{k-j}, 2 k-j}\left(u_{1}, \ldots, u_{k-j}\right) \\
& =\operatorname{Coef}_{2 k-j+1} \ln \left[\frac{1+z u_{1}+\cdots+z^{k} u_{k}}{1+z u_{1}+\cdots+z^{k-j} u_{k-j}}\right]
\end{aligned}
$$




$$
\begin{aligned}
& =\operatorname{Coef}_{2 k-j+1} \ln \left(1+w_{k-j+1} z^{k-j+1}+\cdots+w_{k} z^{k}+\cdots\right) \\
& =\frac{1}{2} \sum_{s=1}^{j} w_{k-j+s} w_{k+1-s}+\cdots
\end{aligned}
$$

Since the quadratic part of this expression is nondegenerate, one can apply the Morse lemma with parameters and get rid of terms of higher degree in w's. See also [47] for an alternative proof of this result and further discussions.

For example, one can check that $W_{\Lambda^{2}, 3}=-u_{1}^{4} / 4+u_{1}^{2} u_{2}-u_{2}^{2} / 2$ and $W_{\Lambda^{1}, 3}=-u_{1}^{4} / 4$, hence

$$
W_{\Lambda^{2}, 3}=-W_{\Lambda^{1}, 3}-\left(u_{2}-u_{1}^{2}\right)^{2} / 2 .
$$

The equation 4.25 can be rewritten as $W_{\Lambda^{k}, 2 k-j}=W_{\Lambda^{k-j}, 2 k-j}+W^{\prime}$, and we get the diagram

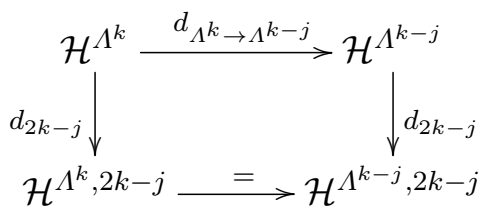

Here the colored differential $d_{\Lambda^{k} \rightarrow \Lambda^{k-j}}$ coincides with the one defined in [44]. It is defined on HOMFLYPT homology colored by $\Lambda^{k}$, and its homology is isomorphic to HOMFLYPT homology colored by $\Lambda^{k-j}$. It corresponds to the "remainder potential" $W^{\prime}=\frac{1}{2} \sum_{s=1}^{j} v_{k-j+s} v_{k+1-s}$ in 4.25. The corresponding Koszul complex is associated to the sequence of polynomials $v_{k-j+1}, \ldots, v_{k}$ :

$$
d_{\Lambda^{k} \rightarrow \Lambda^{k-j}}\left(\xi_{i}\right)=v_{i+k-j},
$$

and its homology is isomorphic to a free superpolynomial algebra generated by $v_{1}, \ldots, v_{k-j}, \xi_{j+1}, \ldots, \xi_{n}$. We can easily see this differential on the picture with the bosonic and fermionic generators labeled by the boxes of a Young diagram. Recall that their $q$-degrees are twice the content and the hooklength of the boxes of $\Lambda^{k}$ respectively, as shown in Figure 15 . The differential $d_{\Lambda^{k} \rightarrow \Lambda^{k-j}}$ has $q$-degree $4 k-2 j$,

$$
(a, q)\left[d_{\Lambda^{k} \rightarrow \Lambda^{k-j}}\right]=(-2,4 k-2 j) .
$$

4.6. Potentials and differentials: extension to $n \lambda$. Let us first fix some notations. Let $\lambda=\left(\lambda_{1}, \ldots, \lambda_{r}\right)$ be a Young diagram. Then the boxes of $n \lambda=\left(n \lambda_{1}, \ldots, n \lambda_{r}\right)$ are split into groups by $n$ boxes labeled by the boxes of $\lambda$. If $x$ is a box of $\lambda$, then we label the corresponding boxes in $n \lambda$ by $x^{(1)}, \ldots, x^{(n)}$.

For every $x$ in $\lambda$ we can also consider the generators $u_{x}$ and $\xi_{x}$ in $\mathcal{H}^{\lambda}$ and the generators $u_{x}^{(i)}$ and $\xi_{x}^{(i)}$ in $\mathcal{H}^{n \lambda}$. For convenience, we can consider 


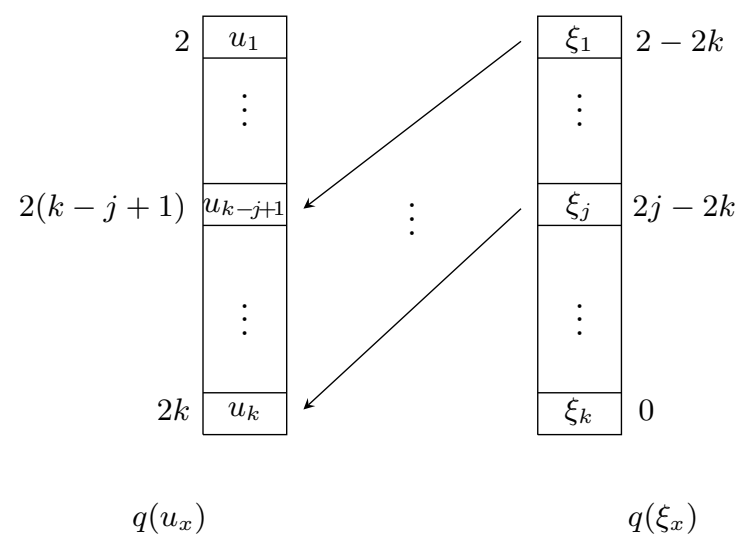

Fig. 15. Action of the differential $d_{\Lambda^{k} \rightarrow \Lambda^{k-j}}$ and the $q$-degrees of the generators $u_{x}, \xi_{x}$ the generating functions

$$
u_{x}(\tau)=\sum_{i=1}^{n} u_{x}^{(i)} \tau^{i-1}, \quad \xi_{x}(\tau)=\sum_{i=1}^{n} \xi_{x}^{(i)} \tau^{i-1} .
$$

Suppose that the diagram $\lambda$ admits a potential $W_{\lambda, N}\left(u_{1}, \ldots, u_{d}\right)$.

CONJECTURE 4.6. For all $n$ the diagrams $n \lambda$ admit potentials $W_{n \lambda, N}$ such that

$$
\widehat{W}(\tau)=\sum_{n=1}^{\infty} \tau^{n-1} W_{n \lambda, N}=W_{\lambda, N}\left(u_{1}(\tau), \ldots, u_{d}(\tau)\right) .
$$

The $\operatorname{sl}(N)$ differential for $n \lambda$ corresponds to $W_{n \lambda, N}$ by 4.23).

Since $W_{\Lambda^{2}, 3}\left(u_{1}, u_{2}\right)=-u_{1}^{4} / 4+u_{1}^{2} u_{2}-u_{2}^{2} / 2$, we have

$$
\begin{aligned}
W_{2 \Lambda^{2}, 3}\left(u_{1}^{(1)}, u_{1}^{(1)}, u_{2}^{(1)},\right. & \left.u_{2}^{(2)}\right)=\operatorname{Coef}_{1}\left[-u_{1}(\tau)^{4} / 4+u_{1}(\tau)^{2} u_{2}(\tau)-u_{2}(\tau)^{2} / 2\right] \\
& =-\left(u_{1}^{(1)}\right)^{3} u_{1}^{(2)}+\left(u_{1}^{(1)}\right)^{2} u_{2}^{(2)}+2 u_{1}^{(1)} u_{1}^{(2)} u_{2}^{(1)}-u_{2}^{(1)} u_{2}^{(2)} .
\end{aligned}
$$

Note that the $u_{i}^{(j)}$ correspond to the boxes of the $2 \times 2$ rectangle (see Figure 16), so that their $q$-degrees are equal to the hook-lengths:

$$
q\left(u_{1}^{(1)}\right)=q\left(u_{1}\right)=2, \quad q\left(u_{1}^{(2)}\right)=4, \quad q\left(u_{2}^{(1)}\right)=q\left(u_{2}\right)=4, \quad q\left(u_{2}^{(2)}\right)=6 .
$$

The potential $W_{2 \Lambda^{2}, 3}$ is homogeneous of $q$-degree 10 .

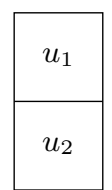

\begin{tabular}{|l|l|}
\hline$u_{1}^{(2)}$ & $u_{1}^{(1)}$ \\
\hline$u_{2}^{(2)}$ & $u_{2}^{(1)}$ \\
\hline
\end{tabular}

Fig. 16. Bosonic generators for $\Lambda^{2}$ and $2 \Lambda^{2}$ 


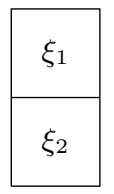

\begin{tabular}{|l|l|}
\hline$\xi_{1}^{(1)}$ & $\xi_{1}^{(2)}$ \\
\hline$\xi_{2}^{(1)}$ & $\xi_{2}^{(2)}$ \\
\hline
\end{tabular}

Fig. 17. Fermionic generators for $\Lambda^{2}$ and $2 \Lambda^{2}$

Moreover, suppose that we have some differential $D$ on $\mathcal{H}^{\lambda}$ given by the formula $D\left(\xi_{l}\right)=p_{l}\left(u_{1}, \ldots, u_{d}\right)$. Such a differential can be naturally extended to $n \lambda$ by

$$
D_{n}\left(\xi_{l}^{(i)}\right)=\operatorname{Coef}_{n-1-i}\left[p_{l}\left(u_{1}(\tau), \ldots, u_{d}(\tau)\right)\right] .
$$

Observe that equations (4.29) and (4.30) agree with each other. Indeed, every potential $W$ induces a differential $D_{W}$ such that $D_{W}\left(\xi_{l}\right)=\frac{\partial W}{\partial u_{l}}$, if we match a bosonic variable $u_{l}$ with the fermionic variable $\xi_{l}$. We can match $u_{l}^{(i)}$ with $\xi_{l}^{(i)}$ so that

$$
\frac{\partial W_{n \lambda, N}}{\partial u_{l}^{(i)}}=\operatorname{Coef}_{n-1}\left[\frac{\partial \widehat{W}}{\partial u_{l}} \frac{\partial u_{l}(\tau)}{\partial u_{l}^{(i)}}\right]=\operatorname{Coef}_{n-1-i}\left[p_{l}\left(u_{1}(\tau), \ldots, u_{d}(\tau)\right)\right] .
$$

As an example of this construction, consider $\lambda=(1)$, so that $n \lambda=(n)$ $=S^{n}$. We have $W_{\lambda, N}=x^{N+1}$, and it was suggested in [39] (see also [40]) that

$$
W_{S^{n}, N}=\operatorname{Coef}_{n-1}\left(x^{(1)}+x^{(2)} \tau+\cdots+x^{(n)} \tau^{n-1}\right)^{N+1} .
$$

This differential differs from the one proposed in [45], but its homology agrees with the known categorifications of the Jones-Wenzl projectors [15, 16, 28].

As a more interesting example, let us compute the colored differential for the $R \times C$ rectangular diagram. Such a diagram can be presented as $C \cdot \Lambda^{R}$, and as such admits an extension of the colored differential $d_{\Lambda^{R} \rightarrow \Lambda^{j}}$. By 4.27) we have

$$
d_{\Lambda^{R} \rightarrow \Lambda^{R-j}}\left(\xi_{l}\right)=u_{l+R-j},
$$

so

$$
d_{R \times C \rightarrow(R-j) \times C}\left(\xi_{l}^{(m)}\right)=u_{l+R-j}^{(m)} .
$$

In Figure 18 we show the action of the differential $d_{2 \Lambda^{2} \rightarrow 2 \Lambda^{1}}$. The left and

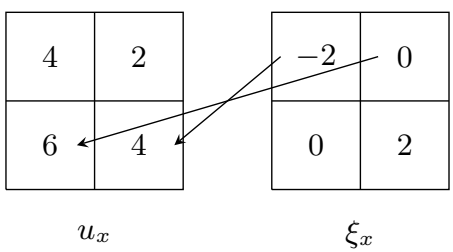

Fig. 18. Colored differential $d_{2 \Lambda^{2} \rightarrow 2 \Lambda^{1}}$ 
right rectangles illustrate the $q$-degrees of the bosonic and fermionic generators respectively. The differential has $q$-degree 6 (the same as $d_{\Lambda^{2} \rightarrow \Lambda^{1}}$ ), and its homology is a free graded supercommutative algebra isomorphic to $\mathcal{H}^{S^{2}}$.

4.7. Hilbert scheme interpretation. In [38, following the earlier work in [37, 40, the authors suggested a relation between braids and coherent sheaves on the Hilbert scheme of points on the plane. Recall that the Hilbert scheme of $n$ points on $\mathbb{C}^{2}$ is the moduli space of ideals $I \subset \mathbb{C}[x, y]$ of codimension $n$. It is a smooth algebraic symplectic variety of dimension $2 n$. It admits a Hamiltonian action of $\mathbb{C}^{*}$ which lifts the Hamiltonian action $t \cdot(x, y)=\left(t^{-1} x, t y\right)$ on $\mathbb{C}^{2}$. The fixed points of this action correspond to monomial ideals and are labeled by Young diagrams of size $n$. The Hilbert scheme carries the tautological bundle $\mathcal{T}$ of rank $n$ whose fiber over a point $I$ is isomorphic to $\mathbb{C}[x, y] / I$. We refer to [69] for all details and further information on Hilbert schemes of points.

Definition 4.7. Given a fixed point $I_{\lambda}$, one can consider the attracting subvariety

$$
Z_{\lambda}:=\left\{I \in \operatorname{Hilb}^{n} \mid \lim _{t \rightarrow \infty} t \cdot I=I_{\lambda}\right\} .
$$

By construction, $Z_{\lambda}$ is a smooth $n$-dimensional subvariety in Hilb ${ }^{n}\left(\mathbb{C}^{2}\right)$ (it is Lagrangian with respect to the symplectic form) isomorphic to $\mathbb{C}^{n}$. The following proposition is well known (see e.g. [69]).

Proposition 4.8. Given an ideal $I$, the limit $\lim _{t \rightarrow \infty} t \cdot I$ exists if and only if I is set-theoretically supported on the line $\{y=0\}$; we denote the set of such ideals by $\operatorname{Hilb}^{n}\left(\mathbb{C}^{2}\right.$, line $)$. In other words,

$$
\operatorname{Hilb}^{n}\left(\mathbb{C}^{2}, \text { line }\right):=\bigcup_{\lambda} Z_{\lambda} .
$$

In short, the main conjecture of [38] can be formulated as follows:

Conjecture 4.9. Given a braid $\beta$ on $n$ strands (or, more generally, an element of the Soergel category categorifying the Hecke algebra), there is an object $\mathcal{F}(\beta) \in \mathcal{D}\left(\mathrm{Hilb}^{n}\left(\mathbb{C}^{2}\right.\right.$, line $\left.)\right)$ such that the HOMFLYPT homology of $\beta$ is isomorphic to the equivariant sheaf cohomology of $\bigoplus_{i=1}^{n} \mathcal{F}(\beta) \otimes \bigwedge^{i} \mathcal{T}^{*}$. The a-grading is equal to $i$, and the differential $d_{M \mid N}$ is given by contraction with the section $s_{M \mid N}$ of $\mathcal{T}$ corresponding to the monomial $x^{M} y^{N}$.

In general, the construction of $\mathcal{F}_{\beta}$ is rather subtle, and we refer the reader to [38] for further details. We are interested in the homology of the unknot colored by a diagram $\lambda$, which corresponds to a categorified Jones-Wenzl projector $\mathcal{P}_{\lambda}$ in the Soergel category. It was argued in [38] that $\mathcal{P}_{\lambda}$ naturally corresponds to the fixed point $I_{\lambda^{t}}$; more precisely, the class $\left[\mathcal{F}\left(\mathcal{P}_{\lambda}\right)\right]$ in the equivariant $\mathrm{K}$-theory is proportional (but not equal) to the class of this fixed 
point. On the other hand, the class $\left[Z_{\lambda^{t}}\right]$ is also proportional to the class $\left[I_{\lambda^{t}}\right]$, so the skyscraper sheaf $\mathcal{O}_{Z_{\lambda t}}$ is naturally related to a certain multiple of the categorified projector $\mathcal{P}_{\lambda}$ (strictly speaking, an object in the triangulated subcategory generated by the direct sums of $\mathcal{P}_{\lambda}$ ). We summarize all these observations in the following:

ConjeCture 4.10. Let $\lambda$ be a rectangular Young diagram. The $\lambda$-colored homology of the unknot (in the normalization used in this paper) corresponds to the sheaf cohomology of $\bigoplus_{i=1}^{n} \mathcal{O}_{Z_{\lambda^{t}}} \otimes \bigwedge^{i} \mathcal{T}^{*}$. The differential $d_{M \mid N}$ is given by contraction with the section $s_{M \mid N}$ of $\mathcal{T}$, corresponding to the monomial $x^{M} y^{N}$.

Proposition 4.11. The colored HOMFLYPT homologies of the unknot described in Conjectures 4.3 and 4.10 agree.

Proof. The $R \times C$ diagram $\lambda$ corresponds to the monomial ideal $I_{\lambda}=$ $\left(x^{C}, y^{R}\right)$. The quotient $\mathbb{C}[x, y] / I_{\lambda}$ is spanned by the monomials $x^{i} y^{j},(i, j)$ $\in \lambda$. The neighborhood of $I_{\lambda}$ in the Hilbert scheme consists of ideals such that the same monomials span the quotient:

$$
I=\left(x^{C}+\sum_{(i, j) \in \lambda} a_{i j} x^{i} y^{j}, y^{R}+\sum_{(i, j) \in \lambda} b_{i j} x^{i} y^{j}\right) .
$$

The $2 C \cdot R$ coefficients $a_{i j}, b_{i j}$ form a natural coordinate system on $\operatorname{Hilb}^{n}\left(\mathbb{C}^{2}\right)$ around $I_{\lambda}$. Under the action of the Hamiltonian torus, $a_{i j}$ has weight $t^{-C+i-j}$ and $b_{i j}$ has weight $t^{R+i-j}$, so

$$
\lim _{t \rightarrow \infty} t \cdot a_{i j}=0, \quad \lim _{t \rightarrow \infty} t \cdot b_{i j}=\infty .
$$

Therefore the attracting subvariety $Z_{\lambda}$ is a vector space spanned by the coordinate vectors corresponding to $a_{i j}$ :

$$
Z_{\lambda}=\left\{I=\left(x^{C}+\sum_{(i, j) \in \lambda} a_{i j} x^{i} y^{j}, y^{R}\right)\right\} .
$$

Furthermore, $\mathcal{T}^{*}$ is trivial on $Z_{\lambda}$ and has a basis $\xi_{i j}$ which is dual to $x^{i} y^{j}$. Therefore

$$
H^{*}\left(\operatorname{Hilb}^{n}\left(\mathbb{C}^{2}, \text { line }\right), \bigoplus_{i=1}^{n} \mathcal{O}_{Z_{\lambda}} \otimes \bigwedge^{i} \mathcal{T}^{*}\right)=\mathbb{C}\left[a_{i j}\right] \otimes \bigwedge\left(\xi_{i j}\right)
$$

Furthermore, this construction agrees with the description of the differentials $d_{N}$.

TheOREM 4.12. If $C=1$ then the homology of $d_{N}$ is isomorphic to the Jacobi ring of $W_{S^{R}, N}$. If $R=1$ then the homology of $d_{N}$ agrees with the Jacobi ring of $W_{\bigwedge^{C}, N}$. 
Proof. If $C=1$ then

$$
Z_{\lambda}=\left\{I=\left(x+\sum_{i=0}^{R-1} a_{i} y^{i}, y^{R}\right)\right\}
$$

The basis $\xi_{i}$ is dual to $y^{i}$. Now $d_{N}\left(\xi_{i}\right)$ is the coefficient of $y^{i}$ in $x^{N}$, but

$$
x^{N}=(-1)^{N}\left(\sum_{i=0}^{R-1} a_{i} y^{i}\right)^{N} \bmod y^{R} .
$$

This agrees (up to sign) with 4.31.

If $R=1$ then

$$
Z_{\lambda}=\left\{I=\left(x^{C}+\sum_{i=0}^{C-1} a_{i} x^{i}, y\right)\right\}
$$

We can write

$$
x^{N}+\sum_{i=0}^{C-1} a_{i}^{(N)} x^{i}=0 \bmod I .
$$

The basis $\xi_{i}$ is dual to $x^{i}$, so $d_{N}\left(\xi_{i}\right)=a_{i}^{(N)}$. For each fixed $i$ one gets a recurrence relation

$$
a_{i}^{(N)}+\sum_{j=0}^{C-1} a_{j} a_{i}^{(N-C+j)}=0, \quad N \geq C,
$$

with initial condition $a_{i}^{(N)}=\delta_{i, N}$ for $N<C$. Therefore

$$
\left(1+\sum_{j=0}^{C-1} a_{j} t^{C-j}\right) \sum_{N=0}^{\infty} t^{N} a_{i}^{(N)}=t^{i}+a_{C-1} t^{i+1}+\cdots+a_{i+1} t^{C-1}
$$

and the generating function for $a_{i}^{(N)}$ is defined by the equation

$$
\sum_{N=0}^{\infty} t^{N} a_{i}^{(N)}=\frac{t^{i}+a_{C-1} t^{i+1}+\cdots+a_{i+1} t^{C-1}}{1+a_{C-1} t+a_{C-2} t^{2}+\cdots+a_{0} t^{C}} .
$$

Finally, let us define $h_{k}\left(a_{0}, \ldots, a_{C-1}\right)$ by the equation

$$
\sum_{k=0}^{\infty} h_{k} t^{k}=\frac{1}{1+a_{C-1} t+a_{C-2} t^{2}+\cdots+a_{0} t^{C}}
$$


then we get

$$
\left\{\begin{array}{l}
a_{C-1}^{(N)}=h_{N-C+1}, \\
a_{C-2}^{(N)}=h_{N-C+2}+a_{C-1} h_{N-C+1}, \\
a_{C-3}^{(N)}=h_{N-C+3}+a_{C-1} h_{N-C+2}+a_{C-2} h_{N-C+1}, \\
\cdots \\
a_{0}^{(N)}=h_{N}+a_{C-1} h_{N-1}+\cdots+a_{1} h_{N-C+1} .
\end{array}\right.
$$

The ideal generated by $d_{N}\left(\xi_{i}\right)=a_{i}^{(N)}$ coincides with the ideal generated by $h_{N-C+1}, \ldots, h_{N}$, and the higher Koszul homologies vanish. This agrees with 4.24): if

$$
W=\operatorname{Coef}_{N+1}\left[\ln \left(1+a_{C-1} t+a_{C-2} t^{2}+\cdots+a_{0} t^{C}\right)\right]
$$

then

$$
\frac{\partial W}{\partial a_{i}}=\operatorname{Coef}_{N+1-C+i} \frac{1}{1+a_{C-1} t+a_{C-2} t^{2}+\cdots+a_{0} t^{C}}=h_{N+1-C+i}
$$

and

$$
\left(\frac{\partial W}{\partial a_{0}}, \ldots, \frac{\partial W}{\partial a_{C-1}}\right)=\left(h_{N-C+1}, \ldots, h_{N}\right)
$$

5. Colored HOMFLYPT homology for torus knots. In this part we describe various algebraic and geometric approaches to the colored HOMFLYPT homology of torus knots.

5.1. Colored HOMFLYPT polynomials of torus knots. Let us first recall the general formula for colored invariants of torus knots 64, 79, 85. Define the coefficients $c_{\lambda, n}^{\mu}$ by the equation

$$
s_{\lambda}\left(x_{1}^{n}, x_{2}^{n}, \ldots\right)=\sum_{|\mu|=n|\lambda|} c_{\lambda, n}^{\mu} s_{\mu}\left(x_{1}, x_{2}, \ldots\right),
$$

where $s_{\lambda}\left(x_{1}, x_{2}, \ldots\right)$ denotes the Schur polynomial. The $\lambda$-colored invariant of the $(n, m)$-torus knot can be expressed via the colored invariants of the unknot by the following formula (we omit an overall scaling factor $\left({ }^{9}\right)$ ):

$$
\bar{P}^{\lambda}(T(n, m))=\sum_{|\mu|=n|\lambda|} q^{-\frac{m}{n} \kappa(\mu)} c_{\lambda, n}^{\mu} \bar{P}^{\mu}(\bigcirc)
$$

where $\kappa(\mu)=\sum_{(i, j) \in \mu}(i-j)=\frac{1}{2} \sum_{j} \mu_{j}\left(\mu_{j}-2 j+1\right)$ is the content of $\mu$.

$\left({ }^{9}\right)$ Throughout this section we rescale the HOMFLYPT polynomials so that the lowest $a$-degree is $a=0$, and the lowest $q$-degree among all nonzero terms with $a=0$ is $q=0$. In other words, the polynomials are rescaled (i.e. multiplied by an overall factor of the form $\left.a^{i} q^{j}\right)$ so that the lowest term has both $a$ - and $q$-degree equal to zero. 
Etingof, the first author and Losev [23] categorified (5.2) as follows. The rational Cherednik algebra $H_{c}\left(S_{n}\right)$ with parameter $c$ is defined by generators $x_{1}, \ldots, x_{n}, y_{1}, \ldots, y_{n}$ and $g \in S_{n}$ and relations

$$
\begin{aligned}
& {\left[x_{i}, x_{j}\right]=0=\left[y_{i}, y_{j}\right], \quad g x_{i} g^{-1}=x_{g(i)}, \quad g y_{i} g^{-1}=y_{g(i)},} \\
& {\left[x_{i}, y_{j}\right]=c(i j) \quad \text { for } i \neq j, \quad\left[x_{i}, y_{i}\right]=1-c \sum_{j \neq i}(i j) .}
\end{aligned}
$$

We refer the reader to [24] and references therein for the detailed information on representation theory of this $H_{c}\left(S_{n}\right)$. Here we just use standard representations $M_{c}(\lambda)$ and simple representations $L_{c}(\lambda)$ labeled by partitions $\lambda$ of size $n$. All these are also graded representations of $S_{n}$.

Let $V$ denote the $(n-1)$-dimensional reflection representation of $S_{n}$. If $W$ is a graded representation of $S_{n}$, then the space $\bigoplus_{j=0}^{n-1} \operatorname{Hom}_{S_{n}}\left(\bigwedge^{j} V, W\right)$ is bigraded by $j$ (which we will refer to as $a$-degree) and by the grading on $W$ (which we will refer to as $q$-degree).

TheOREM 5.1 ([23]).

(a) The (a,q)-bigraded character of $\bigoplus_{j=0}^{n-1} \operatorname{Hom}_{S_{n}}\left(\bigwedge^{j} V, M_{c}(\mu)\right)$ equals $q^{-c \kappa(\mu)} P^{\mu}(\bigcirc)$.

(b) In the Grothendieck group of representations of $H_{c}\left(S_{n d}\right)$ (where $d=|\lambda|$ ),

$$
\left[L_{\frac{m}{n}}(n \lambda)\right]=\sum_{|\mu|=n \lambda} c_{\lambda, n}^{\mu}\left[M_{m / n}(\mu)\right]
$$

where the coefficients $c_{\lambda, n}^{\mu}$ are defined by (5.1).

(c) Consider the space

$$
\mathcal{H}_{m, n}^{\lambda}:=\bigoplus_{j=0}^{n d-1} \operatorname{Hom}_{S_{n d}}\left(\bigwedge^{j} V, L_{m / n}(n \lambda)\right) .
$$

Then the $(a, q)$-graded character of $\mathcal{H}_{m, n}^{\lambda}$ equals $P^{\lambda}(T(n, m))$.

Indeed, part (c) follows from (a) and (b) combined with (5.2). This suggests the following:

Conjecture 5.2. The space $\mathcal{H}_{m, n}^{\lambda}$ admits additional gradings (or filtrations) $t_{r}$ and $t_{c}$, and is isomorphic to $\mathcal{H}^{\lambda}\left(T_{m, n}\right)$.

For $\lambda=\square$, Conjecture 5.2 agrees with the main conjecture of [40], and the filtration was explicitly constructed there. For $\lambda=S^{r}$ we discuss a possible filtration below.

5.2. Stable HOMFLYPT homology of torus knots. We will be interested in the stable limit of the colored HOMFLYPT polynomials of $(n, m)$-torus knots as $m \rightarrow \infty$. 
Proposition 5.3. Let $\kappa(\mu)=\sum_{(i, j) \in \mu}(i-j)$. Define

$$
\bar{P}_{\mathrm{st}}^{\lambda}(T(n, m)):=q^{-m n \kappa(\lambda)+m / n \kappa(n \lambda)} a^{-\frac{m(n-1)|\lambda|}{2}} \bar{P}^{\lambda}(T(n, m)) .
$$

Then

$$
\lim _{m \rightarrow \infty} \bar{P}_{\mathrm{st}}^{\lambda}(T(n, m))=\bar{P}^{n \lambda}(\bigcirc) .
$$

Proof. One can check that in 5.1 one has $c_{\lambda, n}^{n \lambda}=1$, and $\kappa(\mu)<\kappa(n \lambda)$ if $\mu \neq n \lambda$ and $c_{\lambda, n}^{n \lambda} \neq 0$. Therefore by (5.2),

$$
\bar{P}_{\mathrm{st}}^{\lambda}(T(m, n))=\bar{P}^{n \lambda}(\bigcirc)+\cdots,
$$

where the dots denote higher order terms in $q^{m / n}$. In the limit $m \rightarrow \infty$, we get 5.3 .

We would like to use (5.3) as a definition of the stable colored homology of the $(n, \infty)$-torus knot.

ConjeCture 5.4. There exists a limit $\overline{\mathcal{H}}^{\lambda}(n, \infty)=\lim _{m \rightarrow \infty} \overline{\mathcal{H}}^{\lambda}(T(n, m))$ which is isomorphic to $\overline{\mathcal{H}}^{n \lambda}(\bigcirc)$ as a vector space. Moreover, the $\left(a, q, t_{c}\right)$ gradings on these spaces match. Following the conjectures in Section 4 , we suggest that for rectangular diagrams $\lambda$ the space $\overline{\mathcal{H}}^{\lambda}(n, \infty)$ is a free supercommutative algebra with bosonic and fermionic generators labeled by the boxes of $n \lambda$.

Theorem 5.5. For $\lambda=\square$, Conjecture 5.4 holds:

(a) [11, 80, 86] The limit of $\overline{\mathcal{H}}^{\square}(T(n, m))$ as $m \rightarrow \infty$ exists and agrees with the $S^{n}$-colored homology of the unknot.

(b) [50] The $S^{n}$-colored HOMFLYPT homology of the unknot is a free supercommutative algebra with $n$ even and $n$ odd generators.

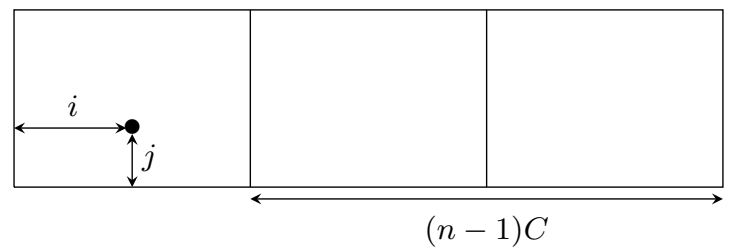

Fig. 19. Generators $u_{i j}^{(n)}$

Let us determine the gradings of the generators of $\overline{\mathcal{H}}^{\lambda}(n, \infty)$. The diagram $p \lambda$ is an $R \times p C$ rectangle, which can be naturally divided into $p$ different $R \times C$ rectangles. Let us denote by $u_{i j}^{(n)}$ a bosonic generator in the $n$th rectangle from the right at box $(i, j)$, and by $\xi_{i j}^{(n)}$ a fermionic generator in the $n$th rectangle from the left at box $(i, j)$, as in Figures 19 and 20. Their 


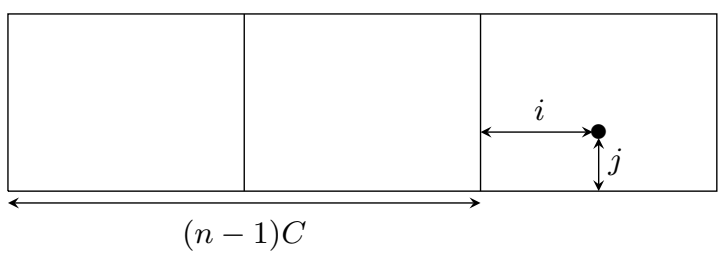

Fig. 20. Generators $\xi_{i j}^{(n)}$

gradings are given by

$$
\begin{aligned}
(a, q)\left[u_{i j}^{(n)}\right] & =(0,2(n C-i+R-j+1)), \\
\left(t_{c}, t_{r}\right)\left[u_{i j}^{(n)}\right] & =(2(n C-i), 2((n-1) R+j-1)), \\
(a, q)\left[\xi_{i j}^{(n)}\right] & =(2,2((n-1) C+i-j)), \\
\left(t_{c}, t_{r}\right)\left[\xi_{i j}^{(n)}\right] & =(2((n-1) C+i)-1,2((n-1) R+j)-1) .
\end{aligned}
$$

Note that $t_{r}$ is constant in rows and $t_{c}$ is constant in columns. This explains the notations $t_{r}$ and $t_{c}$. Using the equation $Q=\left(q+t_{r}-t_{c}\right) / R$, we get

$$
Q\left[u_{i j}^{(n)}\right]=2 n, \quad Q\left[\xi_{i j}^{(n)}\right]=2 n-2 .
$$

It is important to note that these elements generate unreduced stable homology. To work with reduced homology, one should consider $u_{i j}^{(n)}, \xi_{i j}^{(n)}$ for $n \geq 2$ since the subalgebra generated by $u_{i j}^{(1)}, \xi_{i j}^{(1)}$ coincides with the unreduced homology of the unknot.

There exists a map $M_{\lambda}: \mathcal{H}^{\lambda}(T(p, \infty)) \rightarrow \mathcal{H}^{\lambda^{t}}(T(p, \infty))$ such that for any $w$ we have

$$
\left(Q, t_{c}, t_{r}\right)\left[M_{\lambda}(w)\right]=\left(Q, t_{r}, t_{c}\right)[w] .
$$

The map $M_{\lambda}$ is defined by

$$
M_{\lambda}\left(u_{i j}^{(n)}\right):=u_{R-j+1, C-i+1}^{(n)}, \quad M_{\lambda}\left(\xi_{i j}^{(n)}\right):=\xi_{j i}^{(n)} .
$$

One can check that it indeed satisfies (5.4). The refined exponential growth conjecture also holds for the stable homology $\mathcal{H}^{\lambda}(T(p, \infty))$ :

$$
\mathcal{P}^{R \times C}\left(T(p, \infty) ; a, Q, t_{r}\right)=\left(\mathcal{P}^{\Lambda^{R}}\left(T(p, \infty) ; a, Q, t_{r}\right)\right)^{C} .
$$

The exponential growth conjecture follows from the fact that $t_{r}$ is constant in rows: if we consider generators in $\left(a, Q, t_{r}\right)$-gradings, then we get $C$ copies of $\mathcal{H}^{\Lambda^{R}}(T(p, \infty))$ in the same gradings, which in turn implies 5.5.

Let us recall that in $(2.9)$ we conjectured that the self-duality map $\Phi$ can be obtained as follows: there exists a "D0-brane" operator $L_{\lambda}: \mathcal{H}^{\lambda}(K) \rightarrow$ $\mathcal{H}^{\lambda}(K)$ such that

$$
\left(a, q, Q, t_{r}, t_{c}\right)\left[L_{\lambda}\right]=(0, R+C, 2, R, C), \quad \Phi_{\lambda}(x)=L_{\lambda}^{-Q(x)} .
$$


We conjecture that for torus knots colored by $R \times C$ rectangular diagrams $\lambda$ the operator $L_{\lambda}$ coincides with multiplication by $u_{C, 1}^{(2)}$. One can check that it has the prescribed degrees. Moreover, the mirror map $M_{\lambda}$ is chosen in such a way that $M_{\lambda}\left(u_{C, 1}^{(2)}\right)=u_{R, 1}^{(2)}$, and so for rectangular diagrams we have

$$
M_{\lambda} \circ L_{\lambda}=L_{\lambda^{t}} \circ M_{\lambda}
$$

and therefore

$$
M_{\lambda} \circ \Phi_{\lambda}=\Phi_{\lambda^{t}} \circ M_{\lambda} .
$$

This is indeed the required compatibility relation between $M$ and $\Phi$.

Now let us describe the action of colored differentials in this algebraic model. The positive row-removing differentials are given by

$$
d_{R \times C \rightarrow k \times C}^{+}\left(\xi_{i, j}^{(n)}\right)=u_{C+1-i, j-k}^{(n)}, \quad j>k .
$$

The positive column-removing differentials are given by

$$
d_{R \times C \rightarrow R \times l}^{+}\left(\xi_{i, j}^{(n)}\right)=u_{C+l+1-i, j}^{(n)}, \quad i>l .
$$

The negative row-removing differentials are given by

$$
d_{R \times C \rightarrow k \times C}^{-}\left(\xi_{1, k+1}^{(2)}\right)=1 .
$$

The negative column-removing differentials are given by

$$
d_{R \times C \rightarrow R \times l}^{-}\left(\xi_{l+1,1}^{(2)}\right)=1 .
$$

The differentials vanish on all other generators of colored HOMFLYPT homology. One can check that the degrees of these differentials agree with the expected ones.

The duality between positive and negative differentials is partly explained by the identities

$$
\begin{array}{rlrl}
d_{R \times C \rightarrow k \times C}^{+}\left(\xi_{1, k+1}^{(2)}\right) & =u_{C, 1}^{(2)}, & d_{R \times C \rightarrow k \times C}^{-}\left(\xi_{1, k+1}^{(2)}\right) & =1, \\
d_{R \times C \rightarrow R \times l}^{+}\left(\xi_{l+1,1}^{(2)}\right) & =u_{C, 1}^{(2)}, & d_{R \times C \rightarrow R \times l}^{-}\left(\xi_{l+1,1}^{(2)}\right)=1 .
\end{array}
$$

5.3. Differential forms. Let $\lambda$ be an $R \times C$ rectangular Young diagram, and let $K=T(p, q)$ be a $(p, q)$-torus knot. We assume that $\mathcal{H}^{\lambda}(K)$ is a quotient of $\mathcal{H}^{\lambda}(T(p, \infty))$, the latter space being $\left(a, q, t_{c}\right)$-graded isomorphic to the $p \lambda$-colored homology of the unknot. Under this assumption, the multiplicative generators of $\mathcal{H}^{\lambda}(K)$ coincide with the ones of $\mathcal{H}^{\lambda}(T(p, \infty))$, while some additional relations are imposed.

Definition 5.6. The unreduced moduli space $\overline{\mathcal{M}}_{p, q}(r)$ is defined in the affine space with the coordinates $u_{1}, \ldots, u_{r p} ; v_{1}, \ldots, v_{r q}$ by

$$
\left(1+u_{1} z+u_{2} z^{2}+\cdots+u_{r p} z^{r p}\right)^{q}=\left(1+v_{1} z+v_{2} z^{2}+\cdots+v_{r q} z^{r q}\right)^{p},
$$

which should hold at every coefficient of its expansion in powers of $z$. 
Proposition 5.7. The space $\overline{\mathcal{M}}_{p, q}(r)$ can also be defined in the affine space with the coordinates $u_{1}, \ldots, u_{r p}$ by

$\operatorname{Coef}_{k}\left[\left(1+u_{1} z+u_{2} z^{2}+\cdots+u_{r p} z^{r p}\right)^{q / p}\right]=0, \quad r q+1 \leq k \leq r q+r p-1$.

Proof. Let

$$
F(z)=\left(1+u_{1} z+u_{2} z^{2}+\cdots+u_{r p} z^{r p}\right)^{q / p}=\sum_{k=0}^{\infty} f_{k}\left(u_{1}, \ldots, u_{r p}\right) z^{k} .
$$

One can rewrite 5.7 as:

$$
\left(1+u_{1} z+u_{2} z^{2}+\cdots+u_{p r} z^{p r}\right)^{q / p}=1+v_{1} z+v_{2} z^{2}+\cdots+v_{q r} z^{q r} .
$$

This will express $v_{i}$ 's through $u_{j}$ 's as $v_{i}=f_{i}\left(u_{1}, \ldots, u_{r p}\right)$, and leave one with the infinite system of equations

$$
f_{k}\left(u_{1}, \ldots, u_{r p}\right)=0, \quad k>q r .
$$

We need to reduce this infinite system to a finite one by proving that $f_{q r+1}=$ $\cdots=f_{q r+p r-1}=0$ implies $f_{k}=0$ for all $k>q r$. Indeed, observe that

$$
F(z)=U(z)^{q / p} \Rightarrow F^{\prime}(z) \cdot U(z)=\frac{q}{p} F(z) U^{\prime}(z) .
$$

By considering the coefficients of $z^{k-1}$ one gets

$k f_{k}+(k-1) f_{k-1} u_{1}+\cdots+(k-p r) f_{k-p r} u_{p r}=\frac{q}{p}\left(f_{k-1} u_{1}+\cdots+p r f_{k-p r} u_{p r}\right)$.

Now we can prove by induction on $k>q r$ that $f_{k}=0$ given $f_{q r+1}=\cdots=$ $f_{q r+p r-1}=0$. For $k \in\{q r+1, \ldots, q r+p r-1\}$ this is obvious. For $k=q r+p r$ the coefficients of $f_{k-p r} u_{p r}$ on both sides of (5.8) are equal:

$$
k-p r=q r+p r-p r=q r=\frac{q}{p} \cdot p r .
$$

So these terms cancel, and $f_{q r+p r}$ is a linear combination of $f_{q r+p r-1}, \ldots, f_{q r+1}$ and hence vanishes. For $k>q r+p r$ the recursion (5.8) expresses $f_{k}$ as a linear combination of $f_{k-1}, \ldots, f_{k-p r}$, which all vanish by the induction assumption, hence $f_{k}=0$.

Proposition 5.8. The dimension of $\overline{\mathcal{M}}_{p, q}(r)$ equals $r$.

Proof. Indeed, let us forget the scheme structure and study the underlying subset of the affine space. One has $U(z)^{q}=V(z)^{p}$ for coprime $p$ and $q$ iff there exists a polynomial $G(z)$ such that $U(z)=G(z)^{p}, V(z)=G(z)^{q}$. Since $G(z)$ starts from 1 and has degree $r$, we have $r$ parameters at our disposal.

ConjeCture 5.9. The unreduced $S^{r}$-homology of the $(p, q)$-torus knot is the space of differential forms on $\overline{\mathcal{M}}_{p, q}(r)$ :

$$
\overline{\mathcal{H}}^{S^{r}}(T(p, q))=\Omega^{\bullet}\left(\overline{\mathcal{M}}_{p, q}(r)\right) .
$$


As an illustration of this conjecture, let us consider the example of the unknot. We can choose $p=q=1$, so (5.7) takes the form

$$
1+u_{1} z+u_{2} z^{2}+\cdots+u_{r} z^{r}=1+v_{1} z+v_{2} z^{2}+\cdots+v_{r} z^{r} .
$$

Therefore $\overline{\mathcal{M}}_{p, q}(r)=\operatorname{Spec} \mathbb{C}\left[u_{1}, \ldots, u_{r}\right]$, and

$$
\Omega^{\bullet}\left(\overline{\mathcal{M}}_{p, q}(r)\right)=\mathbb{C}\left[u_{1}, \ldots, u_{r}, d u_{1}, \ldots, d u_{r}\right] .
$$

This agrees with the above description of the unreduced $S^{r}$-colored triplygraded homology of the unknot.

Definition 5.10. The reduced moduli space $\mathcal{M}_{p, q}(r)$ is defined in the affine space with coordinates $u_{r+1}, \ldots, u_{p r} ; v_{r+1}, \ldots, v_{q r}$ by all coefficients in the $z$-expansion of the equation

$$
\begin{aligned}
\left(1+u_{r+1} z^{r+1}+u_{r+2} z^{r+2}+\cdots+u_{p r} z^{p r}\right)^{q} & \\
= & \left(1+v_{r+1} z^{r+1}+v_{r+2} z^{r+2}+\cdots+v_{q r} z^{q r}\right)^{p} .
\end{aligned}
$$

Similarly to Proposition 5.8 one can check that $\mathcal{M}_{p, q}(r)$ defines a single point 0 , so it is zero-dimensional. We propose the following:

CONJECture 5.11. The reduced $S^{r}$-homology of the $(p, q)$-torus knot is the space of differential forms on $\mathcal{M}_{p, q}(r)$ :

$$
\mathcal{H}^{S^{r}}(T(p, q))=\Omega^{\bullet}\left(\mathcal{M}_{p, q}(r)\right) .
$$

Conjectures 5.11 and 5.9 are motivated by the following result.

TheOREM $5.12([23,36])$.

(a) $\Omega^{\bullet}\left(\overline{\mathcal{M}}_{p, q}(r)\right) \simeq \mathcal{H}_{q, p}^{S^{r}}$, where the space $\mathcal{H}_{m, n}^{\lambda}$ was introduced in Theorem 5.1 .

(b) The $(a, q)$-character of $\Omega^{\bullet}\left(\overline{\mathcal{M}}_{p, q}(r)\right)$ is equal to the unreduced $S^{r}$-colored HOMFLYPT polynomial of the $(p, q)$-torus knot.

(c) The $(a, q)$-character of $\Omega^{\bullet}\left(\mathcal{M}_{p, q}(r)\right)$ is equal to the reduced $S^{r}$-colored HOMFLYPT polynomial of the $(p, q)$-torus knot.

(d) $\Omega^{\bullet}\left(\overline{\mathcal{M}}_{p, q}(r)\right) \simeq \Omega^{\bullet}\left(\mathcal{M}_{p, q}(r)\right) \otimes \mathbb{C}\left[u_{1}, \ldots, u_{r}, d u_{1}, \ldots, d u_{r}\right]$.

Let us describe the gradings on the space $\Omega^{\bullet}\left(\mathcal{M}_{p, q}(r)\right)$. The $a$-grading is defined by the degree of a differential form, so that $a\left(u_{i}\right)=0, a\left(d u_{i}\right)=2$. Furthermore, we define the $\left(q, t_{r}, t_{c}\right)$-gradings by

$$
\begin{aligned}
\left(q, t_{c}, t_{r}\right)\left[u_{i}\right] & =\left(2 i, 2 i-2,2\left\lfloor\frac{i-1}{r}\right\rfloor\right), \\
\left(q, t_{r}, t_{c}\right)\left[d u_{i}\right] & =\left(2 i-2,2 i-1,2\left\lfloor\frac{i-1}{r}\right\rfloor+1\right) .
\end{aligned}
$$


It is easy to check that the defining equations of $\mathcal{M}_{p, q}(r)$ are homogeneous in $q$-grading and not homogeneous in $t$-gradings. Therefore, strictly speaking, on $\Omega^{\bullet}\left(\mathcal{M}_{p, q}(r)\right)$ we get the $(a, q)$-bigrading and a pair of filtrations $\left(t_{r}, t_{c}\right)$.

For example, in the uncolored case the reduced homology of the trefoil knot has only one bosonic generator $u_{2}$, and the defining equation for $\mathcal{M}_{2,3}(1)$ reads

$$
u_{2}^{2}=0 \text {. }
$$

Similarly, for the $(3,4)$-torus knot the reduced uncolored homology has two bosonic generators $u_{2}$ and $u_{3}$, with the following defining equations for $\mathcal{M}_{3,4}(1)$ :

$$
u_{2} u_{3}=0, \quad \frac{2 u_{2}^{3}}{9}=u_{3}^{2} .
$$

The reduced $S^{2}$-colored homology of the trefoil knot has two even generators $u_{3}$ and $u_{4}$ with the defining equations

$$
u_{3} u_{4}=u_{4}^{2}=u_{3}^{3}=0 .
$$

Their differentials have the form

$$
u_{3} d u_{4}+u_{4} d u_{3}=2 u_{4} d u_{4}=3 u_{3}^{2} d u_{3}=0 .
$$

So one can check that the monomial basis in $\Omega^{*}\left(\mathcal{M}_{2,3}(2)\right)$ is given by

$$
1, u_{3}, u_{4}, u_{3}^{2}, d u_{3}, d u_{4}, u_{3} d u_{3}, u_{3} d u_{4}, d u_{3} \wedge d u_{4} \text {. }
$$

We illustrate this homology in Figure 21 (compare with the figures in Section 3.1.

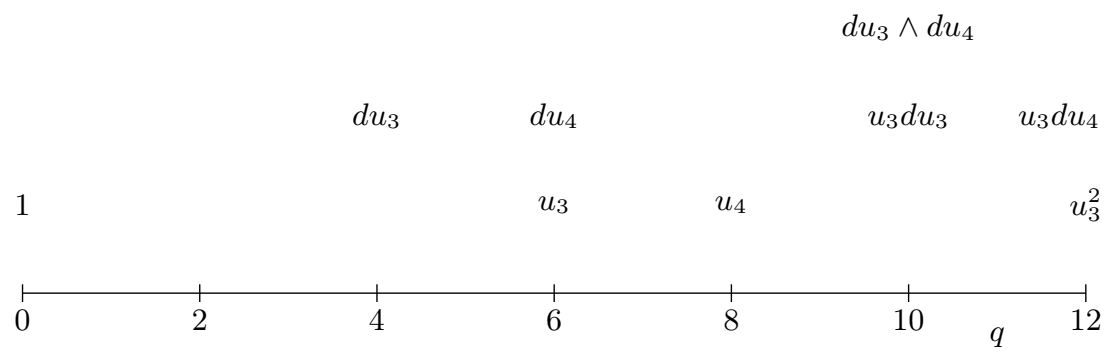

Fig. 21. Algebraic model for the $S^{2}$-homology of the trefoil

For example, by computing the gradings of the basic elements 5.15 one finds the Poincare polynomial of the $S^{2}$-colored trefoil knot (in the $\left(a, q, t_{r}\right)$ grading): $\mathcal{P}^{S^{2}}\left(3_{1}\right)=1+q^{6} t^{2}+q^{8} t^{2}+q^{12} t^{4}+a^{2} q^{4} t^{3}+a^{2} q^{6} t^{3}+a^{2} q^{10} t^{5}+a^{2} q^{12} t^{5}+a^{4} q^{10} t^{6}$. 
More generally, for the reduced $S^{r}$-colored homology of the trefoil knot the $\left(a, q, t_{r}\right)$-gradings of the generators are given by

$$
\left(a, q, t_{r}\right)\left[u_{i}\right]=(0,2 i, 2), \quad\left(a, q, t_{r}\right)\left[d u_{i}\right]=(2,2 i-2,3) .
$$

Conjecture 5.13 ([30, 31, 29]). The Poincaré polynomial of the reduced HOMFLYPT homology of the $S^{r}$-colored trefoil equals

$$
\mathcal{P}^{S^{r}}\left(3_{1}\right)=a^{2 r} q^{-r} \sum_{i+j \leq r} a^{2 j} q^{2(r+1) i+2 r j+j(j-1)} t_{r}^{2 i+3 j} \frac{[r] !}{[i] ![j] ![r-i-j] !} .
$$

Here $[N]$ ! is the unbalanced quantum factorial:

$$
[N] !=[N]^{\prime} \cdot[N-1]^{\prime} \cdot \ldots \cdot[2]^{\prime} \cdot[1]^{\prime}, \quad[N]^{\prime}=\frac{q^{2 N}-1}{q^{2}-1}=1+q^{2}+\cdots+q^{2(N-1)} .
$$

We expect that (5.17) follows directly from Conjecture 5.11, but we do not pursue this combinatorial exercise here. In particular, the monomial $u_{r+1}^{i} \cdot d u_{r+1} \wedge d u_{r+2} \wedge \ldots \wedge d u_{r+j}$ has grading

$i(0,2 r+2,2)+\sum_{l=1}^{j}(2,2 r+2 l-2,3)=(2 j, 2(r+1) i+2 j r+j(j-1), 2 i+3 j)$ and contributes $a^{2 j} q^{2(r+1) i+2 r j+j(j-1)} t_{r}^{2 i+3 j}$ to the Poincare polynomial.

Finally, let us match the gradings 5.12 with those in the construction of Section 4.4. We have $R=1, C=r$, so there are generators $u_{i, 1}^{(n)}, \xi_{i, 1}^{(n)}$, $1 \leq i \leq r$, with gradings

$$
\begin{aligned}
& \left(a, q, t_{c}, t_{r}\right)\left[u_{i, 1}^{(n)}\right]=(0,2+2 r n-2 i, 2 r n-2 i, 2 n-2), \\
& \left(a, q, t_{c}, t_{r}\right)\left[\xi_{i, 1}^{(n)}\right]=(2,2 r(n-1)+2 i-2,2 r(n-1)+2 i-1,2 n-1) .
\end{aligned}
$$

Indeed, these gradings agree with 5.12 if one identifies

$$
u_{i, 1}^{(n)}=u_{r n+1-i}, \quad \xi_{i, 1}^{(n)}=d u_{r(n-1)+i} .
$$

5.4. Differentials for supergroups. It has been conjectured in 35 , Conj. 3.22] that the differentials $d_{0}, d_{ \pm n}$ belong to a bigger algebra. In particular, for the uncolored homology of the $(4,5)$-torus knot the following operators were introduced: $\alpha_{2}$ of $(a, q, t)$-degree $(-2,-2,-5), \alpha_{1}$ of $(a, q, t)$ degree $(-2,2,-3)$. Their introduction was motivated by the observation that the "usual" differentials $d_{N}$ applied to the (unique) generator with top $a$-degree 6 do not generate uncolored triply-graded homology: there are seven nontrivial differentials $d_{0}, d_{ \pm n}$, while there are nine generators with $a$-degree 4 . The extra differentials solve this problem. We can now identify these operators with the supergroup differentials:

$$
\alpha_{2}=d_{1 \mid 2}, \quad \alpha_{1}=d_{2 \mid 1} .
$$


In total, for the uncolored $(4,5)$ knot we have nine nontrivial differentials:

$$
\begin{gathered}
d_{1}=d_{1 \mid 0}, \quad d_{-1}=d_{0 \mid 1}, \\
d_{2}=d_{2 \mid 0}, \quad d_{0}=d_{1 \mid 1}, \quad d_{-2}=d_{0 \mid 2}, \\
d_{3}=d_{3 \mid 0}, \quad \alpha_{1}=d_{2 \mid 1}, \quad \alpha_{2}=d_{1 \mid 2}, \quad d_{-3}=d_{0 \mid 3} .
\end{gathered}
$$

For a general $(n, m)$-torus knot $(m>n)$ we will have all differentials $d_{a \mid b}$ with $a+b<n$, of total number

$$
2+3+4+\cdots+n=n(n+1) / 2-1=(n+2)(n-1) / 2 .
$$

It has been explained in [40] that the differentials are tightly related to the (conjectural) action of the rational Cherednik algebra $H_{c}$ on the triplygraded homology of torus knots. More precisely, [40, Definitions 7.2-7.3] assign a differential to every $S_{n}$-equivariant copy of the standard $(n-1)$ dimensional irreducible representation $V_{n}$ inside $H_{c}$. These differentials have some nice properties: for example, if the two copies $\alpha$ and $\beta$ are pure, then by [40, Lemma 7.7] the corresponding differentials $d_{\alpha}$ and $d_{\beta}$ anticommute.

The new supergroup differentials can be naturally embedded in this framework. Namely, recall that $H_{c}$ has generators $x_{i}, y_{j}$. We define $\alpha(m \mid k)$ to be a copy of $V_{n}$ spanned by $x_{i}^{m} y_{i}^{k}$, and

$$
d_{m \mid k}=d_{\alpha(m \mid k)} .
$$

Since this copy is pure in the sense of [40, the corresponding differentials anticommute. Moreover, one can check that the $\alpha(m \mid k)$ span $\operatorname{Hom}_{S_{n}}\left(V_{n}, H_{c}\right)$, so the supergroup differentials form a complete collection of differentials for uncolored torus knot homology. The grading conventions of [40] ensure that the $d_{m \mid n}$ have the prescribed gradings.

5.5. Examples. We have compared the results of Section 3.1 with the algebraic model in (5.15). Let us make similar comparisons for other torus knots of Section 3 .

The stable reduced $\boxplus$-colored homology of the $(2, \infty)$-torus knot has four even and four odd generators $u_{i j}^{(2)}$ and $\xi_{i j}^{(2)}$ (see Section 5.2. They have the following $\left(a, q, t_{c}, t_{r}\right)$-degrees:

$$
\begin{aligned}
\operatorname{deg}\left[u_{11}^{(2)}\right] & =(0,10,6,4), & \operatorname{deg}\left[u_{12}^{(2)}\right] & =(0,8,6,6), \\
\operatorname{deg}\left[u_{21}^{(2)}\right] & =(0,8,4,4), & \operatorname{deg}\left[u_{22}^{(2)}\right] & =(0,6,4,6), \\
\operatorname{deg}\left[\xi_{11}^{(2)}\right] & =(2,4,5,5), & \operatorname{deg}\left[\xi_{12}^{(2)}\right] & =(2,2,5,7), \\
\operatorname{deg}\left[\xi_{21}^{(2)}\right] & =(2,6,7,5), & \operatorname{deg}\left[\xi_{22}^{(2)}\right] & =(2,4,7,7) .
\end{aligned}
$$

As explained in Section $5.2, Q\left[u_{i j}^{(2)}\right]=4$ and $Q\left[\xi_{i j}^{(2)}\right]=2$. For example, the "bottom row" of the homology (with an appropriate shift of gradings) of the trefoil is a 16-dimensional quotient of this polynomial algebra with monomial basis 


$$
\begin{gathered}
1, u_{21}^{(2)},\left(u_{21}^{(2)}\right)^{2},\left(u_{21}^{(2)}\right)^{3},\left(u_{21}^{(2)}\right)^{4}, \\
u_{11}^{(2)}, u_{11}^{(2)} u_{21}^{(2)}, u_{11}^{(2)}\left(u_{21}^{(2)}\right)^{2}, \\
u_{12}^{(2)}, u_{12}^{(2)} u_{21}^{(2)}, u_{12}^{(2)}\left(u_{21}^{(2)}\right)^{2}, \\
u_{22}^{(2)}, u_{22}^{(2)} u_{21}^{(2)}, u_{22}^{(2)}\left(u_{21}^{(2)}\right)^{2}, \\
\left(u_{22}^{(2)}\right)^{2},\left(u_{11}^{(2)}\right)^{2} .
\end{gathered}
$$

The self-symmetry map $\Phi$ reflects the first four sets of monomials in the vertical axis, and preserves the remaining two monomials.

The stable reduced $\boxplus$-colored homology of the $(2, \infty)$-torus knot has six even and six odd generators. They have the following $\left(a, q, t_{c}, t_{r}\right)$-degrees:

$$
\begin{array}{ll}
\operatorname{deg}\left[u_{11}^{(2)}\right]=(0,12,6,6), & \operatorname{deg}\left[u_{12}^{(2)}\right]=(0,10,6,8), \\
\operatorname{deg}\left[u_{13}^{(2)}\right]=(0,8,6,10), & \operatorname{deg}\left[u_{21}^{(2)}\right]=(0,10,4,6), \\
\operatorname{deg}\left[u_{22}^{(2)}\right]=(0,8,4,8), & \operatorname{deg}\left[u_{23}^{(2)}\right]=(0,6,4,10), \\
\operatorname{deg}\left[\xi_{11}^{(2)}\right]=(2,4,5,7), & \operatorname{deg}\left[\xi_{12}^{(2)}\right]=(2,2,5,9), \\
\operatorname{deg}\left[\xi_{13}^{(2)}\right]=(2,0,5,11), & \operatorname{deg}\left[\xi_{21}^{(2)}\right]=(2,6,7,7), \\
\operatorname{deg}\left[\xi_{22}^{(2)}\right]=(2,4,7,9), & \operatorname{deg}\left[\xi_{23}^{(2)}\right]=(2,2,7,11) .
\end{array}
$$

Indeed, $Q\left[u_{i j}^{(2)}\right]=4$ and $Q\left[\xi_{i j}^{(2)}\right]=2$.

Finally, consider the $S^{2}$-colored $(3,4)$-torus knot. In the notation of Section 5.3, we have four generators $u_{3}, u_{4}, u_{5}, u_{6}$ of $\left(a, q, t_{c}, t_{r}\right)$-gradings

$$
\begin{array}{ll}
\operatorname{deg} u_{3}=(0,6,4,2), & \operatorname{deg} u_{4}=(0,8,6,2), \\
\operatorname{deg} u_{5}=(0,10,8,4), & \operatorname{deg} u_{6}=(0,12,10,4) .
\end{array}
$$

Note that in the notation of Section 5.2. one would have

$$
u_{3}=u_{21}^{(2)}, \quad u_{4}=u_{11}^{(2)}, \quad u_{5}=u_{21}^{(3)}, \quad u_{6}=u_{11}^{(3)},
$$

and the grading conventions agree. The defining relations have the form

$$
\begin{array}{r}
u_{3}^{3}-9 u_{4} u_{5}-9 u_{3} u_{6}=2 u_{3}^{2} u_{4}-3\left(u_{5}^{2}+2 u_{4} u_{6}\right)=u_{3} u_{4}^{2}+u_{3}^{2} u_{5}-3 u_{5} u_{6}=0, \\
5 u_{3}^{4}-12 u_{4}^{3}-72 u_{3} u_{4} u_{5}-36 u_{3}^{2} u_{6}+54 u_{6}^{2}=5 u_{3}^{3} u_{4}-9 u_{4}^{2} u_{5}-9 u_{3}\left(u_{5}^{2}+2 u_{4} u_{6}\right)=0 .
\end{array}
$$

One can check that the monomial basis in the "bottom row" is given by the following 25 monomials:

$$
\begin{gathered}
1, u_{3}, u_{3}^{2}, u_{3}^{3}, u_{3}^{4}, u_{3}^{5}, u_{3}^{6}, \\
u_{4}, u_{4} u_{3}, u_{4} u_{3}^{2}, u_{4} u_{3}^{3}, u_{4} u_{3}^{4}, \\
u_{5}, u_{5} u_{3}, u_{5} u_{3}^{2}, u_{5} u_{3}^{3}, \\
u_{6}, u_{6} u_{3}, u_{6} u_{3}^{2}, u_{6} u_{3}^{3}, \\
u_{4}^{2} u_{4}^{2} u_{3}, u_{4}^{2} u_{3}^{2}, \\
u_{5}^{2}, u_{4}^{3} .
\end{gathered}
$$


The self-symmetry map $\Phi$ reflects the first five sets of monomials in the vertical axis, and preserves the remaining two monomials.

6. HOMFLYPT homology from A-model and B-model. In this section, we wish to look for geometric and physical models for colored HOMFLYPT homology, eventually formulating it in the language of symplectic geometry or the so-called A-model.

Indeed, in mirror symmetry, the so-called B-model is famous for its formulation in the language of complex (algebraic) geometry, while the A-model side involves symplectic invariants, Fukaya category, and quantum cohomology [60]. Clearly, the algebraic model described in the previous section is closer to the B-model, and therefore it will be our natural starting point here. Then, we will relate it to various problems - interesting in their own right - in mirror symmetry and in symplectic geometry. We will give several reformulations, in terms of A-model and B-model, open and closed (i.e. with and without D-branes), in all of which colored HOMFLYPT homology will be realized as cohomology of a suitable BRST operator $Q$ such that $Q^{2}=0$ :

$$
\mathcal{H}^{\lambda}(K)=Q \text {-cohomology }
$$

In physics literature, $Q$ is often called "supercharge" and its cohomology is often called the space of BPS states since its elements are the so-called BPS states (= supersymmetric configurations). Even though precisely this interpretation of knot homology was proposed in [43] and studied from various vantage points in [2, 19, 41, 52, 96], the A-model and B-model reformulations discussed here appear to be completely new and do not make direct contact with any of the existent geometric/physical models of knot homologies. Therefore, by pursuing some of these new geometric descriptions further, one is likely to learn a lot about colored HOMFLYPT homology, and possibly even about homological mirror symmetry.

6.1. B-model on a supermanifold. With this goal in mind, we start by reformulating Conjectures 5.9 and 5.11 in terms of the B-model on a super-manifold $\mathbb{C}^{d \mid d}$ with a certain Landau-Ginzburg potential $W_{\text {super }}(K ; \lambda)$, such that

$$
\overline{\mathcal{H}}^{\lambda}(K) \cong \operatorname{Jac}\left(W_{\text {super }}(K ; \lambda)\right)
$$

Note that $\mathbb{C}^{d \mid d}$ is a super-Calabi-Yau manifold (see [3, 82, 97]).

Specifically, consider the potential $W\left(T(p, q) ; S^{r}\right)$ on the $u$-space given by

$$
W\left(T(p, q) ; S^{r}\right)=\operatorname{Coef}_{(p+q) r+1}\left(1+u_{1} z+\cdots+u_{p r} z^{p r}\right)^{(q+p) / p} .
$$

Let us rename $d u_{i}$ as $\xi_{i}$ and introduce a potential $W_{\text {super }}\left(T(p, q) ; S^{r}\right)$ on the 
superspace $\mathbb{C}^{p r \mid p r}$ with coordinates $u_{i}, \xi_{i}$ by

$$
W_{\text {super }}\left(T(p, q) ; S^{r}\right):=\sum_{i} \frac{\partial W}{\partial u_{i}} \xi_{i} \text {. }
$$

TheOREM 6.1. The space $\mathbb{C}\left[\overline{\mathcal{M}}_{p, q}(r)\right]$ of functions is isomorphic to the Jacobi ring of $W\left(T(p, q) ; S^{r}\right)$. The space $\Omega^{\bullet}\left(\overline{\mathcal{M}}_{p, q}(r)\right)$ of differential forms is isomorphic to the Jacobi ring of $W_{\text {super }}\left(T(p, q) ; S^{r}\right)$ :

$$
\Omega\left[\overline{\mathcal{M}}_{p, q}(r)\right]=\mathbb{C}\left[u_{1}, \ldots, u_{p r}, \xi_{1}, \ldots, \xi_{p r}\right] /\left(\frac{\partial W_{\text {super }}}{\partial u_{i}}, \frac{\partial W_{\text {super }}}{\partial \xi_{i}}\right) .
$$

Proof. Indeed, the partial derivatives of $W\left(T(p, q) ; S^{r}\right)$ are

$$
\begin{aligned}
& \frac{\partial W\left(T(p, q) ; S^{r}\right)}{\partial u_{i}}=\operatorname{Coef}_{(p+q) r+1} \frac{\partial}{\partial u_{i}}\left(1+u_{1} z+\cdots+u_{p r} z^{p r}\right)^{(q+p) / p} \\
& =\frac{q+p}{p} \operatorname{Coef}_{(p+q) r}\left[z^{i}\left(1+u_{1} z+\cdots+u_{p r} z^{p r}\right)^{q / p}\right] \\
& =\frac{q+p}{p} \operatorname{Coef}_{(p+q) r+1-i}\left(1+u_{1} z+\cdots+u_{p r} z^{p r}\right)^{q / p}, \quad i=1, \ldots, p r .
\end{aligned}
$$

Therefore by Proposition 5.7 the ring of functions on $\overline{\mathcal{M}}_{p, q}(r)$ coincides with the Jacobi ring of $W$ :

$$
\mathbb{C}\left[\overline{\mathcal{M}}_{p, q}(r)\right]=\mathbb{C}\left[u_{1}, \ldots, u_{p r}\right] /\left(\frac{\partial W}{\partial u_{i}}\right) .
$$

The full space $\Omega^{\bullet}\left(\overline{\mathcal{M}}_{p, q}(r)\right)$ of differential forms is the quotient of the space of all differential forms on $\mathbb{C}^{p r}$ by the equations

$$
\frac{\partial W}{\partial u_{i}}=0, \quad \sum_{j} \frac{\partial^{2} W}{\partial u_{i} \partial u_{j}} \cdot d u_{j}=0 .
$$

The Jacobi ring of $W_{\text {super }}$ is defined by the following equations:

$$
\frac{\partial W_{\text {super }}}{\partial \xi_{i}}=\frac{\partial W}{\partial u_{i}}=0, \quad \frac{\partial W_{\text {super }}}{\partial u_{i}}=\sum_{j} \frac{\partial^{2} W}{\partial u_{j} \partial u_{i}} \xi_{j}=0 .
$$

Since these equations coincide with (6.7), we get the relation 6.5).

In the mirror symmetry literature [82, 84, 3, 32, the setup we just described is called a Landau-Ginzburg B-model with target space $\mathbb{C}^{d \mid d}$ and superpotential $W_{\text {super }}(K ; \lambda)$. The function $W_{\text {super }}(K ; \lambda)$ is called the superpotential even when the target space is an ordinary (bosonic) manifold, e.g. $W\left(u_{i}\right)$ in our discussion might be called the superpotential of a LandauGinzburg model on $\mathbb{C}^{p r}$. As a final clarification of terminology, we should mention that a model is called Landau-Ginzburg when the superpotential is nonzero, and is referred to as the sigma-model (on a certain target manifold) when $W=0$. 
In our case, the colored HOMFLYPT homology (of torus knots) is realized as the Jacobi ring $\left({ }^{10}\right)$ 6.2 -6.5 of a Landau-Ginzburg model on $\mathbb{C}^{p r \mid p r}$ with the superpotential $W_{\text {super }}\left(T(p, q) ; S^{r}\right)$. There are several aspects of this B-model that make it rather special and interesting, the main being undoubtedly the fact that the target space $\mathbb{C}^{p r \mid p r}$ is a supermanifold.

It is instructive, though, to start with a more familiar and conventional Bmodel with a target space $X$. Then, we will extend it to a Landau-Ginzburg model with a superpotential $W$, and will see why (6.2)-(6.5) can be interpreted as a ring of observables with and without D-branes (called B-branes in the context of the B-model). In general, we shall use $X$ for the target space of the B-model and $Y$ for the target space of the $A$-model, so that $X$ and $Y$ are mirror manifolds in most of our applications.

First, as a warm-up and a lightning review of the relevant facts about the B-model, let us consider a B-model with target space $X$. The space of observables - or, to be more precise, the space of closed (string) observables - in such model is then identified with the $\bar{\partial}$-cohomology of $X$ with values in $\bigwedge^{p} T_{X}$ :

$$
H^{q}\left(X, \bigwedge^{p} T_{X}^{1,0}\right)
$$

Indeed, every $(0, q)$-form on $X$ with values in $\bigwedge^{p} T_{X}$,

$$
A=d \bar{z}^{\bar{k}_{1}} \ldots d \bar{z}^{\bar{k}_{q}} A_{\bar{k}_{1} \ldots \bar{k}_{q}}^{j_{1} \ldots j_{p}} \frac{\partial}{\partial z_{j_{1}}} \ldots \frac{\partial}{\partial z_{j_{p}}},
$$

in the topological B-model defines an observable:

$$
\mathcal{O}_{A}=\eta^{\bar{k}_{1}} \ldots \eta^{\bar{k}_{q}} A_{\bar{k}_{1} \ldots \bar{k}_{q}}^{j_{1} \ldots j_{p}} \theta_{j_{1}} \ldots \theta_{j_{p}} .
$$

Contracting this " $(-p, q)$-form" with the holomorphic $(n, 0)$-form $\Omega$ on $X$ we obtain an isomorphism between this space and $(n-p, q)$-forms, where $n$ is the total (complex) dimension of $X$. Moreover, $\left\{Q, \mathcal{O}_{A}\right\}=-\mathcal{O}_{\bar{\partial} A}$, so that observables in the B-model are Dolbeault cohomologies of forms valued in exterior powers of the holomorphic tangent bundle, i.e. elements of 6.8.

Incorporating a superpotential $W \neq 0$ leads to a Landau-Ginzburg model where the $Q$-operator acts as a differential $Q=\bar{\partial}+Q_{\text {bdry }}$, such that

$$
Q^{2}=W \cdot \mathrm{id} .
$$

As a result, the space of closed string states in the Landau-Ginzburg model can be described as a Hochschild homology, or equivalently as a hypercohomology:

$$
\mathrm{HH}_{*}(\mathrm{MF}(X, W)) \cong \mathbb{H}^{*}\left(\Lambda^{*} \Omega_{X}, d W \wedge\right)
$$

$\left({ }^{10}\right)$ Also known as the "chiral ring" or "ring of observables"; see below. 
where $\Lambda^{*} \Omega_{X}$ denotes the exterior powers of the sheaf of differential forms. The dual cohomology theory is

$$
\mathrm{HH}^{*}(\mathrm{MF}(X, W)) \cong \mathbb{H}^{*}\left(\Lambda^{*} T_{X},[W,-]\right)
$$

where $\Lambda^{*} T_{X}$ denotes the exterior powers of the tangent sheaf and $[W,-]$ denotes the Lie bracket with $W$. In particular, if $f_{1}, \ldots, f_{n}$ are sections of line bundles $\mathcal{E}_{i}$ on a smooth variety $V$, then they define a function $W$ : $\operatorname{Tot}\left(\oplus \mathcal{E}_{i}^{*}\right) \rightarrow \mathbb{C}$ and we have the following equivalence of categories $\left({ }^{11}\right)$.

$$
\operatorname{MF}\left(\operatorname{Tot}\left(\oplus \mathcal{E}_{i}^{*}\right), W\right) \cong \mathrm{QCoh}\left(\left\{f_{i}=0\right\}\right)
$$

that allows us to describe B-branes in a Landau-Ginzburg theory with the superpotential $W$ (i.e. objects in the category of matrix factorizations of $W$ ) in terms of the category of coherent sheaves on the critical variety of $W$.

THEOREM 6.2 ([74]).

$$
H^{0}(M F(W)) \cong \mathcal{D}_{\text {sing }}^{b}\left(W^{-1}(0)\right) .
$$

Note that this brings us very close to our applications (5.9) and (5.11), where the moduli spaces $\overline{\mathcal{M}}_{p, q}(r)$ and $\mathcal{M}_{p, q}(r)$ are defined by polynomial equations $f_{i}\left(u_{j}\right)=0$ in the space $X=\mathbb{C}^{p r}$ of $u$ 's. In situations like this, when the equations $f_{i}\left(u_{j}\right)=0$ integrate to a superpotential $W\left(u_{j}\right)$, the chiral ring (also known as the $(c, c)$ ring) of the Landau-Ginzburg model on $X=\mathbb{C}^{n}$ is isomorphic to the quotient of the ring of polynomial functions $\mathbb{C}\left[u_{1}, \ldots, u_{n}\right]$ by $d W$,

$$
\mathcal{R}_{\text {closed }}\left(\mathbb{C}^{n}, W\right)=\mathbb{C}\left[u_{i}\right] /(d W) .
$$

When $W$ is quasihomogeneous, this ring is graded by the R-charge symmetry $U(1)_{R}$.

In order to interpret the colored HOMFLYPT homology, though, in the Landau-Ginzburg model, not only do we need to find a natural home for the moduli spaces $\overline{\mathcal{M}}_{p, q}(r)$ and $\mathcal{M}_{p, q}(r)$ but also to describe the corresponding spaces of differential forms (5.9) and (5.11) in the B-model language. One way to do this is to replace the target space $\mathbb{C}^{n}$ by the supermanifold $X=\mathbb{C}^{n \mid n}$ and to replace the superpotential $W\left(u_{i}\right)$ by $W_{\text {super }}\left(u_{i} ; \xi_{i}\right)$ defined in 6.4). This gives

$$
\mathcal{R}_{\text {closed }}\left(\mathbb{C}^{n \mid n}, W_{\text {super }}\right)=\Omega^{*}(\mathcal{M})
$$

where $\mathcal{M}=\left\{u \in \mathbb{C} \mid f_{i}(u) \equiv \partial_{i} W(u)=0\right\}$. Specifically, for the superpotential $W_{\text {super }}$ defined by (6.3) and (6.4) we get a B-model realization of the $S^{r}$-colored HOMFLYPT homology of torus knots. For instance, from 5.13

$\left.{ }^{11}\right)$ The analogous statement for the topological A-model is

$$
\operatorname{Fuk}(Y, W) \cong \operatorname{Fuk}(\operatorname{Sing}(W)) .
$$


and 5.14 we find the following potentials in the simple examples considered earlier:

$$
\begin{aligned}
W\left(3_{1} ; \square\right) & =u_{2}^{3}, \quad W\left(8_{19} ; \square\right)=-\frac{7 u_{2}^{4}}{243}+\frac{14 u_{2} u_{3}^{2}}{27}, \\
W_{\text {super }}\left(3_{1} ; \square\right) & =3 u_{2}^{2} \xi_{2}, \quad W_{\text {super }}\left(8_{19} ; \square\right)=\left(-\frac{28 u_{2}^{3}}{243}+\frac{14 u_{3}^{2}}{27}\right) \xi_{2}+\frac{28 u_{2} u_{3}}{27} \xi_{3} .
\end{aligned}
$$

Although conceptually this Landau-Ginzburg model should be the effective two-dimensional $\mathcal{N}=2$ theory on $D$ in the brane construction 4.2 studied in [19, 31, 29], the setup appears to be surprisingly different. Yet, in both cases, the Poincaré polynomial of the colored HOMFLYPT homology is realized as a supersymmetric index (character) of the two-dimensional $\mathcal{N}=2$ theory on $D$. One important difference is that here we have a different $2 \mathrm{~d} \mathcal{N}=2$ theory for each particular value of the color, whereas in [19, 31, 29] a single $2 \mathrm{~d} \mathcal{N}=2$ theory determines $S^{r}$-colored superpolynomials for all values of $r$. It would be interesting to better understand how the new Landau-Ginzburg realization of the colored HOMFLYPT homology discussed here relates to the $2 \mathrm{~d} \mathcal{N}=2$ theory on $D$ studied in [19, 31, 29].

6.2. B-branes and open B-model. There is another way, however, to describe the space of differential forms that is familiar to practitioners of mirror symmetry. This will require introducing one more ingredient in our story, namely D-branes, and considering the space of open string states, i.e. the states of open strings stretched between branes. In the context of the topological B-model, branes are usually called B-branes. Mathematically, B-branes are objects of the derived category of coherent sheaves $\mathcal{D}^{b}(X)$ in the case of the sigma-model (when $W=0$ ), or the category $\operatorname{MF}(X, W)$ of matrix factorizations in the case of Landau-Ginzburg model with target space $X$ and superpotential $W$. We already saw both of these categories in our previous discussion and now we will spend more time discussing their objects, or B-branes.

Note that both the derived category of coherent sheaves and the category of matrix factorizations are also familiar to practitioners of knot homology: these categories play a key role in the constructions of e.g. [57, 58, 92, 98, 100.

Now, let us describe the "open" analogue of 6.8 and 6.16 , i.e. the space of states of open strings ending on a brane $\mathcal{B}$ in the B-model, starting with the sigma-model on $X$ (with $W=0$ ), as we did in our previous discussion, and then extend it to branes in more general Landau-Ginzburg models. In both cases, the conclusion will be that open strings ending on $\mathcal{B}$ form an 
algebra, which is the Ext algebra $\left({ }^{12}\right)$

$$
\mathcal{R}_{\text {open }}(\mathcal{B})=\operatorname{Ext}^{*}(\mathcal{B}, \mathcal{B})
$$

In order to see how it comes about, let us first consider two different branes, $\mathcal{B}_{1}$ and $\mathcal{B}_{2}$, represented by sheaves $\mathcal{E}$ and $\mathcal{F}$ supported on $S \subset X$. Then states of open strings stretched between $\mathcal{B}_{1}$ and $\mathcal{B}_{2}$ are elements of

$$
H^{q} t\left(S, \mathcal{E}^{\vee} \otimes \mathcal{F} \otimes \bigwedge^{p} \mathcal{N}_{S / X}\right) .
$$

There is a spectral sequence with $(6.19)$ as the second page that converges to (see [83])

$$
\operatorname{Ext}_{X}^{p+q}(\mathcal{E}, \mathcal{F})
$$

and in many cases one has

$$
\operatorname{Ext}_{X}^{n}(\mathcal{E}, \mathcal{F}) \cong \bigoplus_{p+q=n} H^{q}\left(S, \mathcal{E}^{\vee} \otimes \mathcal{F} \otimes \bigwedge^{p} \mathcal{N}_{S / X}\right)
$$

because the spectral sequence degenerates at $E_{2}$.

Now let us see how the boundary chiral ring (6.18) can help us to produce yet another interpretation of (5.9) and (5.11) in the open B-model. The simplest example is the unknot colored by $\lambda=S^{r}$, whose HOMFLYPT homology $\overline{\mathcal{H}}^{S^{r}}(\bigcirc)$ is realized as the space of differential forms on $V=$ Spec $\mathbb{C}\left[u_{1}, \ldots, u_{r}\right]$. The corresponding choice of B-brane is closely related to the SYZ brane [87, which plays an important role in mirror symmetry. Namely, it is well known that for a "zero-brane" (i.e. a skyscraper sheaf $\mathcal{O}_{p}$ ) supported at a smooth point on a manifold $X$ of complex dimension $r$ the open string algebra 6.18 is the exterior algebra

$$
\operatorname{Ext}^{*}\left(\mathcal{O}_{p}, \mathcal{O}_{p}\right) \cong \Lambda^{*}(V)
$$

where $V=T_{p} X \cong \Lambda^{*} \mathbb{C}^{r}$. In order to upgrade $\Lambda^{*}(V)$ to the space of differential forms $\Omega^{*}(V)=\mathbb{C}[V] \otimes \Lambda\left(V^{\vee}\right)$, one simply needs to replace $X \cong \mathbb{C}^{r}$ by its complexification $X_{\mathbb{C}} \cong X \times X$, and consider a B-brane $\mathcal{B}=\mathcal{O}_{\Delta}$, where $\Delta \cong \mathbb{C}^{r}$ is the diagonal in $X \times X \cong \mathbb{C}^{r} \times \mathbb{C}^{r}$. Note that the new target space $X \times X \cong \mathbb{C}^{2 r}$ can be viewed as a SYZ torus fibration with a singular fiber at the origin. To facilitate the discussion of the mirror A-model that will follow next, it is convenient to replace $X=\mathbb{C}^{r}$ by $X=\left(\mathbb{C}^{*}\right)^{r}$. Then the target space $X \times X \cong\left(\mathbb{C}^{*}\right)^{2 r}$ is also a $T^{2 r}$ fibration, but without singular fibers, and one has

$$
\mathcal{R}_{\text {open }}(\mathcal{B})=\operatorname{Ext}^{*}\left(\mathcal{O}_{\Delta}, \mathcal{O}_{\Delta}\right) \cong \mathbb{C}\left[u_{i}^{ \pm 1}, d u_{i}\right] .
$$

Before we proceed to the mirror A-model, let us briefly discuss another interesting feature of this example.

$\left({ }^{12}\right)$ The algebra structure comes from the Yoneda product on the self-Ext groups. 
Note that 6.23) can be identified with $\operatorname{HH}^{*}\left(\mathcal{D}^{b}(X)\right) \cong \mathbb{C}\left[u_{i}^{ \pm 1}, d u_{i}\right]$, which, according to our previous discussion, describes closed string states, not open. This is an illustration of a more general phenomenon: the open string states (6.18) of a B-brane supported on the diagonal $\Delta \subset X \times X$ can be identified with the closed string states (6.8) of a B-model on $X$. The mathematical content of this statement is summarized in the following:

Theorem 6.3 (Hochschild-Kostant-Rosenberg isomorphism [49]).

$$
\operatorname{Ext}_{X \times X}^{n}\left(\mathcal{O}_{\Delta}, \mathcal{O}_{\Delta}\right) \cong \bigoplus_{p+q=n} H^{q}\left(X, \bigwedge^{p} T_{X}\right)
$$

where $\Delta$ is the diagonal in $X \times X$.

Moreover, following [60], Yekutieli [99] shows that there is an isomorphism from the hypercohomology to self-Ext:

$$
\mathbb{H}^{n}\left(X, D_{\text {poly }}(X)\right)=\operatorname{Ext}_{X \times X}^{n}\left(\mathcal{O}_{X}, \mathcal{O}_{X}\right)
$$

which is compatible with the cup product on the left and the Yoneda product on the right. And using the isomorphism between Hochschild cohomology and polyvector field cohomology [33], one has (see [61]):

$$
\operatorname{HH}^{*}\left(\mathcal{D}^{b}(X)\right) \cong \operatorname{Ext}_{X \times X}^{*}\left(\mathcal{O}_{\Delta}, \mathcal{O}_{\Delta}\right) \cong H^{*}\left(X, \Lambda^{*} T_{X}\right) .
$$

6.3. HOMFLYPT homology from symplectic geometry. Now, let us see what kind of A-models one gets by applying mirror symmetry to the open and closed B-models in the above discussion.

In general, we denote by $Y$ the mirror of $X$. The simplest example is $X=\left(\mathbb{C}^{*}\right)^{r}$ viewed as a (trivial) $T^{r}$ fibration over $\mathbb{R}^{r}$. Then mirror symmetry is simply a T-duality (a "Fourier transform") along the fibers, and the mirror manifold is $Y \cong T^{*} T^{r}$. The mirror of the statement $\operatorname{HH}^{*}\left(\mathcal{D}^{b}(X)\right) \cong$ $\mathbb{C}\left[u_{i}^{ \pm 1}, d u_{i}\right]$ is the statement about the Hochschild cohomology of the Fukaya category $\mathcal{F}(Y)$,

$$
\mathrm{HH}^{*}\left(\mathcal{F}\left(T^{*} T^{r}\right)\right) \cong \mathbb{C}\left[u_{i}^{ \pm 1}, d u_{i}\right],
$$

which, by analogy with (6.26), can also be realized as the boundary OPE algebra in the A-model of $Y \times \bar{Y}$, where $\bar{Y}$ denotes the space $Y$ with the symplectic form $-\omega$. Indeed, in general, when a symplectic manifold $Y$ has "enough" Lagrangians, the Hochschild cohomology of its Fukaya category $\mathcal{F}(Y)$ is expected [1, 60, 17] to be isomorphic to the Lagrangian Floer cohomology of the (anti)diagonal $\Delta_{Y} \hookrightarrow Y \times \bar{Y}$,

$$
\operatorname{HH}^{*}(\mathcal{F}(Y)) \cong \operatorname{HF}^{*}\left(\Delta_{Y}, \Delta_{Y}\right) .
$$

Thus, in our basic example of 6.23 and 6.27), mirror symmetry maps a B-brane $\mathcal{B}=\mathcal{O}_{\Delta_{X}}$ to a Lagrangian A-brane supported on the conormal 
bundle $T_{\Delta}^{\vee}$ to $T_{\Delta} \subset T^{n} \times T^{n}$ in $Y \times Y=T^{*} T^{r} \times T^{*} T^{r}$, so that

$$
\operatorname{HF}^{*}\left(T_{\Delta}^{\vee}, T_{\Delta}^{\vee}\right) \cong \mathbb{C}\left[u_{i}^{ \pm 1}, d u_{i}\right] .
$$

This is an open A-model version of (6.27) and a concrete illustration of (6.28).

In general, this leads us to the following picture, where the (colored) HOMFLYPT homology is realized either in terms of the closed A-model of a symplectic manifold $Y$,

$$
\mathcal{H}^{S^{r}}(K)=\mathrm{HH}^{*}(\mathcal{F}(Y)),
$$

or in terms of the open A-model (Floer homology) of a certain Lagrangian submanifold $L$,

$$
\mathcal{H}^{S^{r}}(K)=\operatorname{HF}^{*}(L, L) .
$$

(In our previous discussion, $L$ was the (anti)diagonal $\Delta_{Y} \hookrightarrow Y \times \bar{Y}$, but we allow more general Lagrangian submanifolds and more general symplectic manifolds here.) For torus knots, the closed A-model version can be obtained by applying the supermanifold version of mirror symmetry [3, 32, 84] to the B-model in Section 6.1. We plan to return to a systematic study of this mirror symmetry elsewhere.

Here, let us simply point out that even for nontorus knots one might hope to find a realization of the form (6.31). For example, for the figure-eight knot $K=4_{1}$ and $r=1$ a natural guess is

$$
L=\mathbf{S}^{2} \text { with } 3 \text { punctures }
$$

embedded in some symplectic manifold $Y$, such that $\operatorname{HF}^{*}(L, L) \cong H^{*}(L)$.

6.4. "Bottom row" of the colored HOMFLYPT homology. In this part we wish to study the "bottom row" of the colored superpolynomial, defined as

$$
\mathcal{P}_{\text {bottom }}^{\lambda}(q, t):=\lim _{a \rightarrow 0} a^{\#} \mathcal{P}^{\lambda}(a, q, t),
$$

where $a^{\#}$ denotes the appropriate power of $a$, such that the product $a^{\#} \mathcal{P}^{\lambda}(a, q, t)$ contains only nonnegative $a$-degrees starting from zero.

The "bottom row" (6.33) has a simple and beautiful interpretation in terms of instanton/vortex counting. In order to explain the connection with instanton and vortices, based on [19], let us start with a simple vortex counting problem that is easy to do "by hand" and that has all the essential features. Then, we shall upgrade it to the equivariant instanton counting relevant to arbitrary knots and links.

Let $\mathcal{V}_{m}$ be the moduli space of $m$ abelian vortices on a two-dimensional plane, $D=\mathbb{R}^{2}$. In other words, $\mathcal{V}_{m}$ is the moduli space of solutions to the 
PDEs

$$
* F_{A}=i|\phi|^{2}-i t, \quad \bar{\partial}_{A} \phi=0,
$$

where $A$ is a connection on a $U(1)$ bundle of first Chern class $m$, and $t$ is a parameter. These equations describe supersymmetric configurations (BPS states) in a two-dimensional theory on $D$ with $\mathcal{N}=(2,2)$ supersymmetry:

$$
\mathcal{T}_{\text {vortex }}: U(1) \text { gauge theory with a single charged field } \phi .
$$

It is well known that $\mathcal{V}_{m}$ is a Kähler manifold of (real) dimension $2 m$. Namely,

$$
\mathcal{V}_{m}=\operatorname{Sym}^{m}(\mathbb{C}) \equiv \mathbb{C}^{m} / S_{m}
$$

where one can think of coordinates on $\mathcal{V}_{m}$ as vortex positions on $D=\mathbb{R}^{2} \cong \mathbb{C}$.

Since $D$ admits a circle action, one can consider the equivariant character, $\mathrm{Ch}_{q}\left(\mathcal{V}_{m}\right)$, with respect to the rotation group $U(1)_{q}$ acting on $D$. Indeed, $U(1)_{q}$ acts on $\mathcal{V}_{m}$ in a natural way, by equal phase rotations on all factors in the symmetric product 6.36). Moreover, identifying the vortex moduli space $\mathcal{V}_{m}=\operatorname{Sym}^{m}(\mathbb{C})$ with the space of monic polynomials of degree $m$,

$$
f(x)=\prod_{j=1}^{m}\left(x-x_{j}\right)=x^{m}+a_{1} x^{m-1}+\cdots+a_{m},
$$

we immediately deduce the isomorphism $\mathcal{V}_{m} \cong \mathbb{C}^{m}=\left(\left\{a_{1}, \ldots, a_{m}\right\}\right)$ and also learn that $U(1)_{q}$ acts on the space $\mathcal{V}_{m}$ with weights $(1, \ldots, m)$. Therefore, the $U(1)_{q}$-equivariant character of $\mathcal{V}_{m}$ is

$$
\mathrm{Ch}_{q}\left(\mathcal{V}_{m}\right)=\frac{1}{(1-q)\left(1-q^{2}\right) \ldots\left(1-q^{m}\right)},
$$

in which a careful reader will recognize the "bottom row" of the unnormalized $S^{m}$-colored HOMFLYPT polynomial of the unknot $\left({ }^{13}\right)$.

This is not an accident, of course, and the physics setup 4.2 predicts that the "bottom row" of any $\lambda$-colored HOMFLYPT homology of any knot $K$ has a similar interpretation in terms of equivariant vortex counting on $D=\mathbb{R}^{2}$. In order to explain this relation, based on [19], we need to generalize our warm-up example in two important ways: first, we need to introduce the homological $t$-grading, and secondly we need a generalization to arbitrary knots. Both of these problems can be achieved by embedding $D=\mathbb{R}^{2}$ into a larger space $M_{4}=\mathbb{R}^{4}$, and realizing our vortex counting problem on $D$ as a special case of counting solutions to coupled instanton-vortex equations, or equivalently via equivariant instanton counting on the 4-manifold $M_{4}$ in the presence of ramification along $D \subset M_{4}$.

$\left({ }^{13}\right)$ Up to a scaling of variable: $q$ from the $U(1)_{q}$-equivariant character throughout this section goes to $q^{2}$ in the conventions we are using for the HOMFLYPT polynomials and homologies in the rest of the paper. 
However, before we proceed to generalizations, let us stay a bit longer in the world of vortex equations and explore the picture which seems to emerge:

$$
\text { knot } K \rightsquigarrow 2 \text { d vortex theory } \mathcal{T}_{\text {vortex }}(K) \rightsquigarrow \mathcal{V}_{m}(K) \text {, }
$$

so that a suitable version $\mathrm{Ch}_{q, t}\left(\mathcal{V}_{m}\right)$ of the equivariant character computes the bottom row of the colored superpolynomial (6.33) with $\lambda=S^{m}$,

$$
\mathrm{Ch}_{q, t}\left(\mathcal{V}_{m}\right)=\mathcal{P}_{\text {bottom }}^{S^{m}}(q, t)
$$

The details of the construction of the $m$-vortex moduli space $\mathcal{V}_{m}(K)$ in the theory $\mathcal{T}_{\text {vortex }}(K)$ can be found in [19]. As already mentioned earlier, $\mathcal{V}_{m}(K)$ is a Kähler quotient; the definition and the practical computation of its equivariant character $\mathrm{Ch}_{q, t}\left(\mathcal{V}_{m}\right)$ can be found in [72, 70].

Already at this stage the reader might suspect that incorporating the $t$-grading and passing to knot homologies can be addressed by considering equivariant cohomology or K-theory of the vortex moduli space,

$$
H_{U(1)_{q}}^{*}\left(\mathcal{V}_{m}(K)\right)
$$

where the $t$-grading is identified with the homological grading. Although this idea is somewhat naive, it is actually on the right track, and it is instructive to pursue it a little further.

Therefore, as the natural next step let us consider a nonabelian generalization of 6.34 that describes supersymmetric solutions in the $2 \mathrm{~d}$ theory:

(6.42) $\mathcal{T}_{\text {vortex }}: U(p+1)$ gauge theory with $p+1$ fundamental fields $\phi_{i}$.

The corresponding vortex equations

$$
\begin{aligned}
* F_{A} & =i \sum_{i=1}^{p+1} \phi_{i} \phi_{i}^{\dagger}-i t, \\
\bar{\partial}_{A} \phi_{i} & =0
\end{aligned}
$$

involve a $U(p+1)$ gauge connection and $p+1$ Higgs fields $\phi_{i}$ in the fundamental representation of the gauge group. Again, the $m$-vortex moduli space is a Kähler manifold of real dimension

$$
\operatorname{dim} \mathcal{V}_{m}^{U(p+1)}=2 m(p+1) .
$$

In particular, the single-vortex moduli space is well known to be a $(2 p+2)$ dimensional space

$$
\mathcal{V}_{1}^{U(p+1)} \cong \mathbb{C} \times \mathbb{C} \mathbf{P}^{p},
$$

where the factor $\mathbb{C}$ is parametrized by the "center-of-mass" position, while $\mathbb{C P}^{p}$ encodes the "internal degrees of freedom" of a single nonabelian vortex. The rotation symmetry $U(1)_{q}$ acts on $\mathbb{C}$ with weight 1 , so we expect that it contributes to the equivariant character a factor $\frac{1}{1-q}$. However, unlike our first example (related to the unknot), now the moduli space $\mathcal{V}_{1}^{U(p+1)}$ has 
nontrivial topology, and therefore, if the $t$-grading is simply the homological grading on 6.41), the suitable generalization of the equivariant character should be a product of $\frac{1}{1-q}$ and the Poincaré polynomial of $\mathbb{C} \mathbf{P}^{p}$,

$$
\frac{1}{1-q} \sum_{i=0}^{p} t^{2 i} .
$$

This turns out to be, up to a change of variables $\left({ }^{14}\right)$ and an overall shift, the correct answer for the bottom row of the (uncolored) HOMFLYPT homology of the $(2,2 p+1)$-torus knot.

Now, following [19, let us explain why all these examples "work" and where the connection with vortex equations comes from. The reason, in fact, is already contained in Section 4.1 where we reviewed the interpretation (4.1) of homological knot invariants in terms of supersymmetric configurations (BPS states) in the string theory setup (4.2). What this interpretation tells us is that specializing to the bottom row (6.33), i.e. taking the limit $a \rightarrow 0$, means the "large volume limit". Indeed, according to the identification of the parameters (4.3), in this limit the interesting geometry (and topology) of the Calabi-Yau space $X$ is replaced by the simplest Calabi-Yau 3-fold, namely the flat space:

$$
X \stackrel{a \rightarrow 0}{\rightsquigarrow} \mathbb{C}^{3} .
$$

In other words, the bottom row of the colored HOMFLYPT homology is described by a much simpler "toy model" of 4.2 in which the pair $\left(X, L_{K}\right)$ is replaced by $\left(\mathbb{C}^{3}, L_{K}\right)$. (Not much happens to the Lagrangian submanifold $L_{K}$ in this limit.)

The next step is to look at this system from the vantage point of the 4-manifold $M_{4} \cong \mathbb{R}^{4}$ and the defect (called the "surface operator" [46]) supported on $D \cong \mathbb{R}^{2}$. Before we took the limit $a \rightarrow 0$, the compactification on $X$ produced abelian gauge theory on $M_{4}$ with gauge group $U(1)$ [55]. Incorporating an extra brane in this setup means including the so-called surface operator supported on $D$, or, mathematically speaking, ramification in the gauge theory on $M_{4}$.

Let $\mathcal{M}_{k, m}$ be the moduli space of abelian instantons on $M_{4}=\mathbb{R}^{4}$ with ramification along $D \subset M_{4}$. In other words, $\mathcal{M}_{k, m}$ is the moduli space of solutions to the self-duality equation on $M_{4} \backslash D$,

$$
F_{A}^{+}=0
$$

with the second Chern class $k$, with monopole number $m=\frac{1}{2 \pi} \int_{D} F_{A}$, and with the prescribed behavior along $D$. The latter is what we call the ramification data; e.g. a simple example of the so-called tame ramification can

$$
\left.{ }^{14}\right) q \rightarrow q^{2}, t \rightarrow t q^{2} .
$$


be obtained by introducing a $\delta$-function source in the self-duality equation 6.48) on $M_{4}$ :

$$
F_{A}^{+}=2 \pi \alpha\left(\delta_{D}\right)^{+} .
$$

The gauge connections which solve this equation have a first-order pole at $D$. More generally, one can study solutions to self-duality equations with singularities of arbitrarily high order,

$$
A=d z\left(\frac{\alpha_{1}}{z}+\frac{\alpha_{2}}{z^{2}}+\cdots\right)+d \bar{z}(\ldots) .
$$

This is called wild ramification and is precisely how the dependence on the knot $K$ will enter, via the choice of the ramification data $\left({ }^{15}\right)$. Let us denote the corresponding moduli space by $\mathcal{M}_{k, m}(K)$.

Much like the vortex moduli space $\mathcal{V}_{m}$ enjoyed an action of the rotation group $U(1)_{q}$, the moduli space of "ramified instantons" on $M_{4} \backslash D \cong \mathbb{C} \times \mathbb{C}^{*}$ has an action of the rotation group $U(1)_{q} \times U(1)_{t}$, where $U(1)_{q}$ acts by rotations of $D=\mathbb{C}$, and $U(1)_{t}$ acts by rotations of its normal bundle $\mathbb{C}^{*}$.

Then, in the limit 6.47) the physical interpretation 4.1) of the colored HOMFLYPT homology predicts $\left({ }^{16}\right)$

$$
\mathrm{Ch}_{q, t}\left(\mathcal{M}_{0, m}(K)\right)=\overline{\mathcal{P}}_{\text {bottom }}^{S^{m}}\left(K ; q^{1 / 2}, t\right),
$$

which is very close to 6.40, and in fact is exactly the sought-after proper generalization of it. The connection with vortex equations is now easy to see. At least for some knots the moduli space $\mathcal{M}_{k, m}(K)$ can be equivalently described as the moduli space of solutions to the coupled instanton-vortex equations

$$
\begin{aligned}
F_{A}^{+} & =2 \pi \phi \otimes \phi^{\dagger}\left(\delta_{D}\right)^{+}, \\
*_{D} F_{B} & =i \phi^{\dagger} \otimes \phi-i t \mathbf{1}_{E}, \\
\bar{\partial}_{A, B} \phi & =0,
\end{aligned}
$$

where $A$ is a unitary connection on the line bundle $\mathcal{L}$ over $M_{4}, B$ is a unitary connection on the bundle $E$ over $D \subset M_{4}, \phi$ is an element $\phi \in$ $H^{0}\left(\operatorname{Hom}\left(E,\left.\mathcal{L}\right|_{D}\right)\right)$, and $\phi^{\dagger}$ is its adjoint.

As far as we know, the instanton-vortex equations 6.52 are new and have not appeared in the literature previously. Their closest cousin is the set of so-called coupled vortex equations studied e.g. in [8, 9], where both

$\left({ }^{15}\right)$ For instance, it was conjectured [19] that $(2,2 p+1)$-torus knots correspond to wild ramification of order $p$.

$\left.{ }^{16}\right)$ We recall that $\overline{\mathcal{P}}^{S^{m}}(K ; a, q, t)$ on the right-hand side is the Poincare polynomial of the $S^{m}$-colored HOMFLYPT homology of the knot $K$. Its physical definition can be found in [4], and computation in various examples, apart from this paper, can be found in $[2,3,21,30,31,29,44,52,71$. 
$E$ and $\mathcal{L}$ are bundles over $D$. In other words, our equations 6.52 can be viewed as analogs of the coupled vortex equations where one of the bundles is extended over $M_{4}$. For instance, for the $(2,2 p+1)$-torus knot $T^{2,2 p+1}$ one takes $E$ to be a unitary bundle of rank $p+1$.

Then, as before, to get the "bottom row" of unreduced $S^{m}$-colored HOMFLYPT homology we need to consider the equivariant character of $\mathcal{M}_{0, m}(K)$ with instanton number $k=0$ and vortex number $m$. In situations where $\mathcal{M}_{k, m}(K)$ can be identified with the moduli space of solutions to 6.52 this boils down to studying nonabelian vortex equations on $D$. Thus, for $2 p+1$ torus knots we end up with $U(p+1)$ vortex equations, so that the equivariant character of $\mathcal{M}_{0, m}\left(T^{2,2 p+1}\right) \cong \mathcal{V}_{m}^{U(p+1)}$ essentially consists of $p+1$ copies of the abelian vortex character that we discussed earlier (cf. (6.38)).

Our prediction is that the equivariant character of the $m$-vortex moduli space $\mathcal{V}_{m}^{U(p+1)}$ matches the "bottom row" of the $S^{m}$-colored HOMFLYPT homology of the $(2,2 p+1)$-torus knot, i.e.

$$
\begin{aligned}
& \mathrm{Ch}_{q, t}\left(\mathcal{V}_{m}^{U(p+1)}\right)=\frac{q^{-p m}}{(q ; q)_{m}} \sum_{0 \leq k_{p} \leq k_{p-1} \leq \cdots \leq k_{1} \leq m}\left[\begin{array}{l}
m \\
k_{1}
\end{array}\right]\left[\begin{array}{c}
k_{1} \\
k_{2}
\end{array}\right] \ldots\left[\begin{array}{c}
k_{p-1} \\
k_{p}
\end{array}\right] \\
& \times q^{(2 m+1)\left(k_{1}+\cdots+k_{p}\right)-\sum_{i=1}^{p} k_{i-1} k_{i}} t^{2\left(k_{1}+\cdots+k_{p}\right)},
\end{aligned}
$$

with the convention $k_{0}:=m$ (see e.g. [29] for the RHS of the above equation). For example, when $m=2$ and $p=1, \mathrm{Ch}_{q, t}\left(\mathcal{V}_{2}^{U(2)}\right)$ can be computed using the equivariant $U(2)$ action on $\mathcal{V}_{2}^{U(2)}$ (see e.g. [19] and [88]):

$$
\mathrm{Ch}_{q, t}\left(\mathcal{V}_{2}^{U(2)}\right)=q^{-2} \frac{1+q^{3} t^{2}+q^{4} t^{2}+q^{6} t^{4}}{(1-q)\left(1-q^{2}\right)}
$$

and the right-hand side matches the Poincaré polynomial of the HOMFLYPT homology of the $(2,3)$-torus knot (= trefoil knot $3_{1}$ ) colored by $\lambda=\square$, as predicted. In the above example $(6.53)$ of the $S^{2}$-colored trefoil, the corresponding moduli space $\mathcal{V}_{2}^{U(2)}$ of two $U(2)$ vortices has been studied in the literature [48, 25, 4, 26, 5] and is known to have two strata, which correspond to separated vortices and coincident vortices. The first stratum - of complex dimension 4 -is simply the product of two single-vortex moduli spaces $\mathcal{V}_{1}^{U(2)}$, modulo the permutation of the two vortices,

$$
\mathcal{U}_{1}\left(\mathcal{V}_{2}^{U(2)}\right) \cong \mathbb{C} \times \frac{\mathbb{C} \times \mathbb{C P}^{1} \times \mathbb{C P}^{1}}{\mathbb{Z}_{2}}
$$

with the "diagonal" removed. The second stratum - of complex dimension 3 is known to be the space of Hecke modifications (times the center of mass position),

$$
\mathcal{U}_{2}\left(\mathcal{V}_{2}^{U(2)}\right) \cong \mathbb{C} \times \mathbb{W} \mathbb{C} \mathbf{P}_{(1,1,2)}^{2}
$$


See [44] for color graphics illustrating the bottom row of $S^{2}$-colored HOMFLYPT homology and the role of $\mathbb{W} \mathbb{C} \mathbf{P}_{(1,1,2)}^{2}$ in its construction.

Another direction for future work, already mentioned in Section 6.1, is the relation between the theory $\mathcal{T}_{\text {vortex }}(K)$ and the surface operator theory considered in [31, 29]. Indeed, here, in Section 6.1, and in [31, 29] the Poincaré polynomial of colored HOMFLYPT homology (known as the superpolynomial) is realized as a certain index (or character) of a two-dimensional $\mathcal{N}=2$ theory that describes the physics on $D$ in the brane construction 4.2 . Yet, the key difference is that here and in Section 6.1 there is a different $\mathcal{N}=2$ theory for each choice of "color" $\lambda$; the index of such theories determines colored superpolynomials, and a priori all such theories are unrelated. In [31, 29], on the other hand, a single $\mathcal{N}=2$ theory $\mathcal{T}_{K}$ (that depends only on the knot $K$ ) determines the entire tower of $S^{m}$-colored homological invariants, via specializations of its supersymmetric index:

$$
\overline{\mathcal{P}}_{K}^{S^{m}}(a, q, t)=\mathcal{I}_{\mathcal{T}_{K}}\left(x=q^{m} ; a, q, t\right) .
$$

Moreover, recursion relations found in [31, 71, 29], which have a natural meaning $\left({ }^{17}\right)$ in the $\mathcal{N}=2$ theory $\mathcal{T}_{K}$, relate equivariant characters of the form $\mathrm{Ch}_{q, t}\left(\mathcal{M}_{0, m}(K)\right)$ to different values of the "vortex number" $m=\frac{1}{2 \pi} \int_{D} F$. Similarly, from the vantage point of Section 6.1, the recursion relations of [31, 71, 29] relate B-models to target manifolds of different (super) dimension in a way reminiscent of the notions of "endoscopy" and "transfer" in the geometric Langlands correspondence [27]. Thus, in the case of the trefoil knot we have

$$
\mathcal{P}_{\text {bottom }}^{S^{m+2}}-\left(q^{-1}-t^{2} q^{m}+(1+q) q^{2 m} t^{2}\right) \mathcal{P}_{\text {bottom }}^{S^{m+1}}+t^{4} q^{3 m-1}\left(q^{m}-1\right) \mathcal{P}_{\text {bottom }}^{S^{m}}=0 .
$$

\section{Appendix A. Rectangle-colored invariants of $(2, m)$-torus knots.} As we use the quantum invariants of $(2, m)$-torus knots colored by representations labeled by rectangular Young diagrams in many examples throughout the paper, we would like to provide the reader with a handy formula for them. To compute $\bar{P}^{\lambda}(T(n, m))$, one just has to determine the "plethysm" coefficients $c_{\lambda, n}^{\mu}$ appearing in (5.1). There is no closed formula for them for general $\lambda, \mu$ and $n$. However, for $n=2$ and $\lambda$ being an $R \times C$ rectangle, a simple formula for the coefficients $c_{\lambda, n}^{\mu}$ was found in [10].

A Young diagram $\mu=\left(\mu_{1}, \ldots, \mu_{2 R}\right)$ is called $(C, R)$-balanced if

$$
\mu_{i}+\mu_{2 R+1-i}=2 C \quad \text { for all } 1 \leq i \leq R .
$$

Balanced diagrams are in 1-to-1 correspondence with splittings of the $C \times R$

$\left({ }^{17}\right)$ E.g. the characteristic variety of the corresponding $q$-difference equations has a nice interpretation as the moduli space of SUSY parameters, etc. 
rectangle into pairs of complementary diagrams, as shown in Figure 22 .

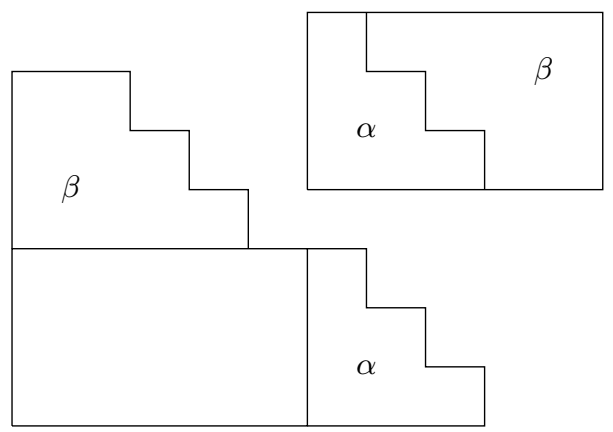

Fig. 22. Diagram $\mu$ is balanced if diagrams $\alpha$ and $\beta$ fill the rectangle.

For a $(C, R)$-balanced diagram $\mu$, let $\operatorname{sgn}(\mu)=(-1)^{|\beta|}=(-1)^{\sum_{i=1}^{R} \mu_{i}}$. Let $B(C, R)$ denote the set of $(C, R)$-balanced diagrams. By [10, Theorem 1],

$$
s_{\lambda}\left(x_{1}^{2}, x_{2}^{2}, \ldots\right)=\sum_{\mu \in B(C, R)}(-1)^{\operatorname{sgn}(\mu)} s_{\mu}\left(x_{1}, x_{2}, \ldots\right),
$$

hence

$$
c_{\lambda, 2}^{\mu}= \begin{cases}(-1)^{\operatorname{sgn}(\mu)} & \text { if } \mu \in B(C, R), \\ 0 & \text { otherwise. }\end{cases}
$$

The equations 5.2 and A.1 determine the invariant $\bar{P}^{\lambda}(T(2, m))$ completely.

Appendix B. A proposal for the (2,1)-colored trefoil. The reduced $\square$-colored HOMFLYPT polynomial of the trefoil is given by

$$
\begin{aligned}
P^{\boxplus}\left(3_{1}\right)(a, q)= & -a^{12}+a^{10}\left(q^{-6}+q^{-4}+q^{-2}+q^{2}+q^{4}+q^{6}\right) \\
& +a^{8}\left(-q^{-10}-2 q^{-6}-3 q^{-2}-3 q^{2}-2 q^{6}-q^{10}\right) \\
& +a^{6}\left(q^{-10}+2 q^{-6}-q^{-4}+2 q^{-2}+2 q^{2}-q^{4}+2 q^{6}+q^{10}\right) .
\end{aligned}
$$

This polynomial has 31 terms, and therefore any homology theory categorifying it should have at least 31 generators. This already indicates the distinction between rectangular and hook diagrams: this time, already for the trefoil, the exponential growth property cannot be satisfied (the uncolored homology of the trefoil is 3 -dimensional and $31>3^{3}$ ). However, some of the structural properties still exist in colored HOMFLYPT homology theories for hook-shaped Young diagrams.

The representations corresponding to $\lambda=\boxminus$ and $\lambda=\square$ have the same dimension and the same (up to scaling) R-matrices of the quantum versions, 
as the representation of any of the superalgebras $\mathrm{gl}(2 \mid 0)$, as $\mathrm{gl}(1 \mid 1)$ and as $\operatorname{gl}(0 \mid 2)$. Therefore, we expect three colored differentials, $d_{2 \mid 0}^{\text {col }}, d_{1 \mid 1}^{\text {col }}$ and $d_{0 \mid 2}^{\text {col }}$ on $\mathcal{H}^{\boxplus}(K)$, such that

$$
\begin{aligned}
& H^{*}\left(\mathcal{H}^{\boxplus}(K), d_{2 \mid 0}^{\text {col }}\right) \cong \mathcal{H}^{\square}(K), \\
& H^{*}\left(\mathcal{H}^{\boxplus}(K), d_{1 \mid 1}^{\text {col }}\right) \cong \mathcal{H}^{\square}(K), \\
& H^{*}\left(\mathcal{H}^{\boxplus}(K), d_{0 \mid 2}^{\text {col }}\right) \cong \mathcal{H}^{\square}(K) .
\end{aligned}
$$

The $(a, q, t)$-degrees of these three differentials are $\operatorname{deg} d_{2 \mid 0}^{\text {col }}=(-2,4,-1), \quad \operatorname{deg} d_{1 \mid 1}^{\text {col }}=(-2,0,-3), \quad \operatorname{deg} d_{0 \mid 2}^{\text {col }}=(-2,-4,-5)$.

The requirement that $\mathcal{H}^{\boxplus}\left(3_{1}\right)$ should categorify $P^{\boxplus}\left(3_{1}\right)(a, q)$, together with the existence of the colored differentials $d_{2 \mid 0}^{\text {col }}, d_{1 \mid 1}^{\text {col }}$ and $d_{0 \mid 2}^{\text {col }}$, essentially determines the $\boxplus$-colored homology of the trefoil knot. It has 41 generators and its Poincaré polynomial is given by

$$
\begin{array}{r}
(\mathrm{B} .5) \mathcal{P} \mathcal{P}_{\left(3_{1}\right)(a, q, t)=a^{12} t^{13}+a^{10}\left(q^{-6} t^{8}+q^{-4} t^{8}+q^{-2} t^{10}+t^{10}+t^{11}+q^{2} t^{12}\right.} \\
\left.+q^{4} t^{12}+q^{6} t^{14}\right)+a^{8}\left(q^{-10} t^{3}+2 q^{-6} t^{5}+q^{-4} t^{5}+q^{-4} t^{6}+3 q^{-2} t^{7}+t^{7}+t^{8}+3 q^{2} t^{9}+q^{4} t^{9}\right. \\
\left.+q^{4} t^{10}+2 q^{6} t^{11}+q^{10} t^{13}\right)+a^{6}\left(q^{-10}+2 q^{-6} t^{2}+q^{-4} t^{3}+2 q^{-2} t^{4}+t^{4}+t^{5}\right. \\
\left.+2 q^{2} t^{6}+q^{4} t^{7}+2 q^{6} t^{8}+q^{10} t^{10}\right) .
\end{array}
$$

An additional nice property that this homology satisfies, and which also exists in the rectangular case, is mirror symmetry. Since $\Xi^{t}=\boxplus$, this becomes a symmetry on $\mathcal{H}^{\boxplus}\left(3_{1}\right)$ :

$$
M_{\square}: \mathcal{H}_{i, j, k}^{\boxminus}\left(3_{1}\right) \rightarrow \mathcal{H}_{i,-j, k-j}^{\boxplus}\left(3_{1}\right),
$$

with the property that

$$
M_{\boxminus} d_{n \mid m}=d_{m \mid n} M_{\boxminus} \cdot
$$

There are some properties that we predict in the case of rectangular representations and which do not exist for the hook diagram. First of all, already for the trefoil, the exponential growth property is not satisfied. The homology is 41-dimensional, and the $\square$-colored HOMFLYPT polynomial already has 31 terms.

Second, this time the isomorphisms $($ B.1 $-(\sqrt{B .3})$ are valid only after an appropriate collapse of tri-grading, i.e. after setting $a=q^{n-m}$ in the case of the differential $d_{n \mid m}$. Additionally, there is no explicit re-grading in these isomorphisms, and we could not find the second $t$-grading. Therefore the theory that we propose is triply-graded. 
Finally, we note that there exists the SL(2) Lefschetz-like action on the homology (B.5) with raising operator of degree $(0,4,2)$. According to the "dictionary" (4.4), this raising operator corresponds to forming a bound state with a BPS state of D0-brane charge $n=4$.

\section{Appendix C. Unreduced colored HOMFLYPT homology}

C.1. General structure. In Section 2, we gave a large list of structural properties that should hold on reduced HOMFLYPT homology. Here we briefly discuss which of these properties should extend to the unreduced case. We first show which of these properties extend to the case of the unknot. Using the assumption that the Poincaré polynomials of the reduced and unreduced homologies are related by

$$
\overline{\mathcal{P}}^{\lambda}(K)=\mathcal{P}^{\lambda}(K) \overline{\mathcal{P}}^{\lambda}(\bigcirc),
$$

we then conclude that the properties that hold both for the reduced homology of any knot and for the unreduced homology of the unknot also hold for the unreduced homology of an arbitrary knot.

In order to simplify the presentation, we focus on the symmetric representations $\lambda=S^{r}$.

Some of the properties of reduced homology extend to the unreduced case: these include the existence and the behavior of the positive-colored differentials and the totally refined exponential growth property. By C.1 these properties will follow from the fact that the unreduced homology of the unknot satisfies these properties.

The unreduced colored homology of the unknot is described in Section 4.2 in terms of the bosonic and fermionic generators. Their explicit $\left(a, Q, t_{r}, t_{c}\right)$ gradings have been obtained, which gives the following Poincaré polynomial of the tilde-version of the unreduced $S^{r}$-colored homology of the unknot:

$$
\widetilde{\overline{\mathcal{P}}}^{S^{r}}(\bigcirc)\left(a, Q, t_{r}, t_{c}\right)=a^{-r} Q^{r} \prod_{i=1}^{r} \frac{1+a^{2} t_{r} t_{c}^{2 i-1}}{1-Q^{2} t_{c}^{2 i-2}} .
$$

In "standard" $\left(a, q, t_{r}, t_{c}\right)$-gradings we have (the same convention, $Q=q+$ $t_{r}-t_{c}$, holds for the symmetric representations as in the case of reduced homology):

$$
\overline{\mathcal{P}}^{S^{r}}(\bigcirc)\left(a, q, t_{r}, t_{c}\right)=a^{-r} q^{r} \prod_{i=1}^{r} \frac{1+a^{2} q^{2 i-2} t_{r} t_{c}^{2 i-1}}{1-q^{2 i} t_{c}^{2 i-2}} .
$$

The degrees of the positive-colored differentials are as in the reduced case:

$$
\left(a, q, t_{r}, t_{c}\right)\left[d_{1 \mid k}^{\mathrm{col}}\right]=(-2,2(1-k),-1,-2 k-1), \quad 0 \leq k<r .
$$


They also have the "colored" property, i.e.

$$
H^{*}\left(\overline{\mathcal{H}}^{S^{r}}(K), d_{1 \mid k}^{\text {col }}\right) \cong \overline{\mathcal{H}}^{S^{k}}(K), \quad 0 \leq k<r .
$$

Indeed, the latter is a consequence of the following lemma (proven by straightforward computation) which shows that (C.5) holds for the unknot and with the re-gradings in the corresponding isomorphisms being exactly the same as for reduced homology (Section 2.5):

Lemma C.1. For all nonnegative integers $r$ and $k$, with $k<r$, we have

$$
\begin{aligned}
\widetilde{\overline{\mathcal{P}}}^{S^{r}}(\bigcirc)\left(a, Q, t_{r}, t_{c}\right)-\widetilde{\overline{\mathcal{P}}}^{S^{k}}(\bigcirc)\left(a, Q t_{c}^{r-k}, t_{r}, t_{c}\right) \\
=\left(1+a^{-2} Q^{2} t_{r}^{-1} t_{c}^{-2 k-1}\right) X
\end{aligned}
$$

for some Laurent polynomial $X\left(a, Q, t_{r}, t_{c}\right)$ all of whose coefficients are nonnegative integers, i.e. $X \in \mathbb{Z}_{+}\left[a^{ \pm 1}, Q^{ \pm 1}, t_{r}^{ \pm 1}, t_{c}^{ \pm 1}\right]$.

The totally refined exponential growth property also holds for the unreduced homology of the unknot. Indeed,

$$
\begin{aligned}
\widetilde{\widetilde{\mathcal{P}}}^{S^{r}}(\bigcirc)\left(a, Q, t_{r}=t, t_{c}=1\right) & =\prod_{i=1}^{r} \frac{1+a^{2} t}{1-Q^{2}} \\
& =\left(\widetilde{\mathcal{\mathcal { P }}}^{\square}(\bigcirc)\left(a, Q, t_{r}=t, t_{c}=1\right)\right)^{r} .
\end{aligned}
$$

On the other hand, there are some differences between the reduced and unreduced homology of any knot.

The first clear distinction from the reduced case is that unreduced homology is infinite-dimensional, since so is the unreduced homology of the unknot. In particular, there can be no symmetry that inverts the $Q$ - or $q$-grading, and therefore the self-symmetry is not satisfied for unreduced homology. Consequently, there are no negative-colored differentials in this case (recall that self-symmetry exchanges positive- and negative-colored differentials).

The second distinction from the reduced homology case is that all differentials $d_{N \mid 0}$, for $N>0$, are now nontrivial, even for the unknot. This is the reason why there is no simple relation like (C.1) between unreduced and reduced $\operatorname{sl}(N)$ homology. In the next section we compute the $\operatorname{sl}(N)$-colored HOMFLYPT homology for some simple knots.

C.2. The unreduced $\mathrm{sl}(N)$-colored HOMFLYPT homology. The unreduced $\left(\operatorname{sl}(N), S^{r}\right)$-colored homology of a knot $K$, denoted $\overline{\mathcal{H}}^{\mathrm{sl}(N), S^{r}}(K)$, is obtained as the homology of $\overline{\mathcal{H}}^{S^{r}}(K)$ with respect to $d_{N \mid 0}$, followed by collapsing of grading by setting $a=q^{N}$. We view $\overline{\mathcal{H}}^{S^{r}}(K)$ here as a triplygraded theory in $\left(a, q, t_{c}\right)$-gradings, and also to simplify notation we denote $t_{c}$ just by $t$. Therefore, $\overline{\mathcal{H}}^{\text {sl( } N), S^{r}}(K)$ becomes a doubly-graded theory in $(q, t)$-degrees. 
Unlike the reduced homology case, here all differentials $d_{N \mid 0}$, with $N>0$, are highly nontrivial, even for the unknot. Below we compute sl(2) homologies for the fundamental and the second-symmetric representations of the unknot, the trefoil and the figure-eight knots.

The $(a, q, t)$-degrees of the $\operatorname{sl}(N)$ differentials $d_{N \mid 0}, N>0$, are as for reduced homology:

$$
(a, q, t)\left[d_{N \mid 0}\right]=(-2,2 N,-1), \quad N>0 .
$$

For all three knots we assume that $d_{N \mid 0}$ is such that it cancels all pairs of generators of $\overline{\mathcal{H}}^{S^{r}}(K)$ whose $(a, q, t)$-degrees differ by $(-2,2 N,-1)$.

C.2.1. Unknot. In the fundamental representation, the Poincaré polynomial of unreduced uncolored HOMFLYPT homology is

$$
\overline{\mathcal{P}}^{\square}(\bigcirc)(a, q, t)=a^{-1} q \frac{1+a^{2} t}{1-q^{2}} .
$$

Then the homology with respect to $d_{n \mid 0}$ is finite-dimensional for any $n$ :

$$
\left(\overline{\mathcal{H}}^{\square}(\bigcirc), d_{n \mid 0}\right)=a^{-1} q\left(1+q^{2}+\cdots+q^{2(n-1)}\right) .
$$

Finally, after collapsing the tri-grading by setting $a=q^{n}$, we get the familiar expression for the uncolored $\operatorname{sl}(N)$ homology of the unknot:

$$
\begin{aligned}
\overline{\mathcal{P}}^{\mathrm{sl}(N), \square}(\bigcirc)(q, t) & =\left(\overline{\mathcal{H}}^{\square}(\bigcirc), d_{N \mid 0}\right)_{\left.\right|_{a=q^{N}}} \\
& =q^{1-N}+q^{3-N}+\cdots+q^{N-1} .
\end{aligned}
$$

The unreduced $S^{2}$-colored homology of the unknot is given by

$$
\overline{\mathcal{P}}^{\square}(\bigcirc)=a^{-2} q^{2} \frac{\left(1+a^{2} t\right)\left(1+a^{2} q^{2} t^{3}\right)}{\left(1-q^{2}\right)\left(1-q^{4} t^{2}\right)} .
$$

To further simplify computation of the homology with respect to $d_{2 \mid 0}$ in terms of the Poincare polynomial, we say that $P_{1}$ is equal to $P_{2}$ modulo $d_{2 \mid 0}$ if $P_{1}-P_{2}=\left(1+a^{-2} q^{4} t^{-1}\right) Y$ for some Laurent polynomial $Y$ in variables $a, q$ and $t$ all of whose coefficients are nonnegative integers.

Then by using the result from the uncolored case, we deduce that modulo $d_{2 \mid 0}$ the expression C.11 equals

$$
\begin{aligned}
a^{-2} q^{2}(1+ & \left.q^{2}\right) \frac{1+a^{2} q^{2} t^{3}}{1-q^{4} t^{2}} \\
& =a^{-2} q^{2}\left(1+q^{2}+q^{4} t^{2} \frac{1+a^{2} t}{1-q^{4} t^{2}}+\left(1+a^{-2} q^{4} t^{-1}\right) \frac{a^{2} q^{2} t^{3}}{1-q^{4} t^{2}}\right) .
\end{aligned}
$$

Therefore

$$
\left(\overline{\mathcal{H}}^{\square}(\bigcirc), d_{2 \mid 0}\right)=a^{-2} q^{2}\left(1+q^{2}+q^{4} t^{2} \frac{1+a^{2} t}{1-q^{4} t^{2}}\right),
$$


and so

$$
\overline{\mathcal{P}}^{\mathrm{sl}(2), \square}(\bigcirc)(q, t)=\left(\overline{\mathcal{H}}^{\square}(U), d_{2 \mid 0}\right)_{\left.\right|_{a=q^{2}}}=q^{-2}+1+q^{2} t^{2} \frac{1+q^{4} t}{1-q^{4} t^{2}} .
$$

C.2.2. Trefoil. For the trefoil, we have

$$
\overline{\mathcal{P}}^{\square}\left(3_{1}\right)=\mathcal{P}^{\square}\left(3_{1}\right) \overline{\mathcal{P}}^{\square}(\bigcirc)=\left(a^{2} q^{-2}+a^{2} q^{2} t^{2}+a^{4} t^{3}\right) a^{-1} q \frac{1+a^{2} t}{1-q^{2}} .
$$

Now after taking the homology with respect to $d_{2 \mid 0}$ which cancels all pairs of generators which differ in $(a, q, t)$-degree by $(-2,4,-1)$, we are left with

$$
\left(\overline{\mathcal{H}}^{\square}\left(3_{1}\right), d_{2 \mid 0}\right)=a\left(q^{-1}+q+q^{3} t^{2}\right)+a^{3} q^{3} t^{3} ;
$$

after setting $a=q^{2}$ this gives

$$
\overline{\mathcal{P}}^{\mathrm{sl}(2), \square}\left(3_{1}\right)(q, t)=\left.\left(\overline{\mathcal{H}}^{\square}\left(3_{1}\right), d_{2 \mid 0}\right)\right|_{a=q^{2}}=q+q^{3}+q^{5} t^{2}+q^{9} t^{3} ;
$$

which equals precisely the free part of the (unreduced) Khovanov homology of the trefoil.

For the second-symmetric representation, we have

$$
\overline{\mathcal{P}}^{\square}\left(3_{1}\right)=\mathcal{P}^{\square}\left(3_{1}\right) \overline{\mathcal{P}}^{\square}(\bigcirc),
$$

where the reduced homology of the trefoil was computed in Section 3 :

$\mathcal{P}^{\square}\left(3_{1}\right)=a^{4}\left(q^{-4}+q^{2} t^{4}+q^{4} t^{6}+q^{8} t^{8}\right)+a^{6}\left(t^{5}+q^{2} t^{7}+q^{6} t^{9}+q^{8} t^{11}\right)+a^{8} q^{6} t^{12}$.

As we have seen above, the Poincaré polynomial of the unknot $\overline{\mathcal{P}}^{\square}(\bigcirc)$ modulo $d_{2 \mid 0}$ equals

$$
\left(\overline{\mathcal{H}}^{\square}(\bigcirc), d_{2 \mid 0}\right)=a^{-2}\left(q^{2}+q^{4}+q^{6} t^{2} \frac{1+a^{2} t}{1-q^{4} t^{2}}\right) .
$$

As for the reduced $S^{2}$-colored Poincaré polynomial of the trefoil, $\mathcal{P} \square\left(3_{1}\right)$, we split it according to the canceling differential $d_{0 \mid 2}^{\text {col }}$ :

$$
\begin{aligned}
\mathcal{P} \square\left(3_{1}\right)= & \left(1+a^{2} q^{4} t^{5}\right)\left(a^{4} q^{-4}+a^{4} q^{2} t^{4}+a^{4} q^{4} t^{6}+a^{6} q^{2} t^{7}\right) \\
& +a^{4} q^{8} t^{8} .
\end{aligned}
$$

Multiplying the RHS of C.18 by $1+a^{2} q^{4} t^{5}$ gives

$$
\begin{aligned}
(1+ & \left.a^{2} q^{4} t^{5}\right) a^{-2}\left(q^{2}+q^{4}+q^{6} t^{2} \frac{1+a^{2} t}{1-q^{4} t^{2}}\right) \\
= & \left(1+a^{2} q^{4} t^{5}\right) a^{-2}\left(q^{2}+q^{4}\right)+a^{-2} q^{6} t^{2}\left(1+a^{2} q^{4} t^{5}\right) \frac{1+a^{2} t}{1-q^{4} t^{2}} \\
= & \left(1+a^{2} q^{4} t^{5}\right) a^{-2}\left(q^{2}+q^{4}\right) \\
& +a^{-2} q^{6} t^{2}\left(1+a^{2} t+q^{4} t^{2}+a^{2} q^{4} t^{3}+\left(1+a^{-2} q^{4} t^{-1}\right) a^{2} q^{4} t^{5} \frac{1+a^{2} t}{1-q^{4} t^{2}}\right)
\end{aligned}
$$




$$
\begin{aligned}
= & a^{-2} q^{2}\left(1+q^{2}+q^{4} t^{2}+a^{2} q^{4} t^{3}+a^{2} q^{6} t^{5}+a^{2} q^{8} t^{5}\right) \\
& +\left(1+a^{-2} q^{4} t^{-1}\right)\left(q^{6} t^{5}+q^{10} t^{7} \frac{1+a^{2} t}{1-q^{4} t^{2}}\right) .
\end{aligned}
$$

Substituting this into C.16, we see that $\overline{\mathcal{P}}^{\square}\left(3_{1}\right)$ modulo $d_{2 \mid 0}$ equals

$$
\begin{array}{r}
a^{-2} q^{2}\left(1+q^{2}+q^{4} t^{2}+a^{2} q^{4} t^{3}+a^{2} q^{6} t^{5}+a^{2} q^{8} t^{5}\right)\left(a^{4} q^{-4}+a^{4} q^{2} t^{4}+a^{4} q^{4} t^{6}+a^{6} q^{2} t^{7}\right) \\
+a^{2} q^{8} t^{8}\left(q^{2}+q^{4}+q^{6} t^{2} \frac{1+a^{2} t}{1-q^{4} t^{2}}\right)
\end{array}
$$

By canceling all possible remaining pairs of generators by $d_{2 \mid 0}$ we get

$$
\begin{aligned}
\left(\overline{\mathcal{H}} \square\left(3_{1}\right), d_{2 \mid 0}\right)= & a^{2}\left(q^{-2}+1\right)+a^{2} q^{2} t^{2}+a^{4} q^{2} t^{3}+a^{2}\left(q^{4}+q^{6}\right) t^{4} \\
& +a^{4}\left(q^{4}+q^{6}\right) t^{5}+a^{4}\left(q^{6}+q^{8}\right) t^{7}+a^{2} q^{10} t^{8}+a^{4} q^{10} t^{9} \\
& +a^{4} q^{12} t^{11}+a^{6} q^{12} t^{12}+a^{2} q^{6} t^{6}\left(1+q^{2}+q^{4} t^{2} \frac{1+a^{2} t}{1-q^{4} t^{2}}\right) .
\end{aligned}
$$

Finally, by setting $a=q^{2}$, we obtain the following $\left(\operatorname{sl}(2), S^{2}\right)$-colored homology of the trefoil:

$$
\begin{aligned}
\mathcal{P}^{\mathrm{sl}(2), \square}\left(3_{1}\right)(q, t)= & \left.\left(\overline{\mathcal{H}}^{\square}\left(3_{1}\right), d_{2 \mid 0}\right)\right|_{a=q^{2}} \\
= & \left(q^{2}+q^{4}\right)+q^{6} t^{2}+q^{10} t^{3}+\left(q^{8}+q^{10}\right) t^{4}+\left(q^{12}+q^{14}\right) t^{5} \\
& +\left(q^{14}+q^{16}\right) t^{7}+q^{14} t^{8}+q^{18} t^{9}+q^{20} t^{11}+q^{24} t^{12} \\
& +q^{10} t^{6}\left(1+q^{2}+q^{4} t^{2} \frac{1+q^{4} t}{1-q^{4} t^{2}}\right) .
\end{aligned}
$$

We note that this result for the free part of the homology coincides (up to an overall shift) with the one obtained in [15] $\left({ }^{18}\right)$.

C.2.3. Figure-eight knot. Similarly, for the figure-eight knot,

$$
\begin{aligned}
\overline{\mathcal{P}}^{\square}\left(4_{1}\right) & =\mathcal{P}^{\square}\left(4_{1}\right) \overline{\mathcal{P}}^{\square}(\bigcirc) \\
& =\left(a^{2} t^{2}+q^{-2} t^{-1}+1+q^{2} t+a^{-2} t^{-2}\right) a^{-1} q \frac{1+a^{2} t}{1-q^{2}} .
\end{aligned}
$$

Taking homology with respect to $d_{2 \mid 0}$ (i.e. modulo $1+a^{-2} q^{4} t^{-1}$ ), we get

$$
\left(\overline{\mathcal{H}}^{\square}\left(4_{1}\right), d_{2 \mid 0}\right)=a^{2} q t^{2}+q^{-1}\left(t^{-1}+1\right)+q(1+t)+a^{-2} q^{-1} t^{-2},
$$

and after setting $a=q^{2}$, we are left with

$$
\left.\left(\overline{\mathcal{H}}^{\square}\left(4_{1}\right), d_{2 \mid 0}\right)\right|_{a=q^{2}}=q^{5} t^{2}+q t+q+q^{-1}+q^{-1} t^{-1}+q^{-5} t^{-2},
$$

$\left({ }^{18}\right)$ Note that the so(3) homology corresponding to the fundamental representation that is computed in $\left[15\right.$ is indeed isomorphic to the homology $\mathcal{H}^{\mathrm{sl}(2), \square}$, due to the well-known isomorphism between the fundamental (vector) representation of so(3) and the second-symmetric representation of $\mathrm{sl}(2)$. 
which is the free part of the (unreduced) Khovanov homology of the figureeight knot.

For the second-symmetric representation we have

$$
\overline{\mathcal{P}}^{\square}\left(4_{1}\right)=\mathcal{P}^{\square}\left(4_{1}\right) \overline{\mathcal{P}}^{\square}(\bigcirc),
$$

where the reduced $S^{2}$-homology is already computed in Section 3 .

$$
\begin{aligned}
\mathcal{P} \square\left(4_{1}\right)= & 1+\left(1+q^{2} t^{2}\right) a^{-2} q^{-2} t^{-4}\left(1+a^{2} q^{-2} t\right)\left(1+a^{2} q^{4} t^{5}\right) \\
& +a^{-4} q^{-4} t^{-8}\left(1+a^{2} q^{-2} t\right)\left(1+a^{2} t^{3}\right)\left(1+a^{2} q^{4} t^{5}\right)\left(1+a^{2} q^{6} t^{7}\right) .
\end{aligned}
$$

In the same way as above for the trefoil, after grouping all terms with the factor $1+a^{2} q^{4} t^{5}$ and multiplying by the RHS of (C.18), we find that modulo $d_{2 \mid 0}$ the polynomial $\overline{\mathcal{P}}^{\square}\left(4_{1}\right)$ equals

$$
\begin{aligned}
& a^{-2} q^{2}\left(1+q^{2}+q^{4} t^{2}+a^{2} q^{4} t^{3}+a^{2} q^{6} t^{5}+a^{2} q^{8} t^{8}\right)\left(\left(1+q^{2} t^{2}\right)\left(1+a^{2} q^{-2} t\right) a^{-2} q^{-2} t^{-4}\right. \\
& \left.+a^{-4} q^{-4} t^{-8}\left(1+a^{2} q^{-2} t\right)\left(1+a^{2} t^{3}\right)\left(1+a^{2} q^{6} t^{7}\right)\right)+a^{-2}\left(q^{2}+q^{4}+q^{6} t^{2} \frac{1+a^{2} t}{1-q^{4} t^{2}}\right) .
\end{aligned}
$$

Finally, by canceling by $d_{2 \mid 0}$ all possible remaining pairs of generators we get

$$
\begin{aligned}
\left(\overline{\mathcal{H}}^{\square}\left(4_{1}\right), d_{2 \mid 0}\right)= & a^{-4}\left(t^{-4}+\left(q^{2}+q^{4}\right) t^{-2}+q^{6}\right) \\
& +a^{-2}\left(t^{-3}+2 q^{2} t^{-1}+q^{4}\left(t^{-1}+t\right)+2 q^{6} t+q^{8} t^{3}\right) \\
& +\left(q^{2}+\left(q^{4}+q^{6}\right) t^{2}+q^{8} t^{4}\right)+a^{-6} q^{-2} t^{-8} \\
& +a^{-4}\left(q^{-2} t^{-7}+t^{-5}+q^{2} t^{-3}+q^{4} t^{-1}\right) \\
& +a^{-2}\left(t^{-4}+q^{2} t^{-2}+2 q^{4}+q^{6} t^{2}+q^{8} t^{4}\right) \\
& +\left(q^{4} t+q^{6} t^{3}+q^{8} t^{5}+q^{10} t^{7}\right)+a^{2} q^{10} t^{8} \\
& +a^{-2} q^{2}\left(1+q^{2}+q^{4} \frac{1+a^{2} t}{1-q^{4} t^{2}}\right) .
\end{aligned}
$$

After setting $a=q^{2}$ in the above expression, we get the ( $\left.\operatorname{sl}(2), S^{2}\right)$-colored homology of the figure-eight knot:

$$
\begin{aligned}
\mathcal{P}^{\mathrm{sl}(2), \square}\left(4_{1}\right)(q, t)= & \left.\left(\overline{\mathcal{H}}^{\square}\left(4_{1}\right), d_{2 \mid 0}\right)\right|_{a=q^{2}} \\
= & q^{-14} t^{-8}+q^{-10} t^{-7}+q^{-8} t^{-5}+\left(q^{-8}+q^{-4}\right) t^{-4} \\
& +\left(q^{-6}+q^{-4}\right) t^{-3}+\left(q^{-6}+q^{-4}+q^{-2}\right) t^{-2} \\
& +\left(q^{-4}+2 q^{-2}+1\right) t^{-1}+\left(q^{-2}+2+q^{2}\right) \\
& +\left(1+2 q^{2}+q^{4}\right) t+\left(q^{2}+q^{4}+q^{6}\right) t^{2}+\left(q^{4}+q^{6}\right) t^{3} \\
& +\left(q^{4}+q^{8}\right) t^{4}+q^{8} t^{5}+q^{10} t^{7}+q^{14} t^{8} \\
& +q^{-2}+1+q^{2} t^{2} \frac{1+q^{4} t}{1-q^{4} t^{2}} .
\end{aligned}
$$

Again, the homology obtained matches the result from [15]. 
C.3. Comparison with the algebraic model. Following Section 4.6 and [39], we can compare the above computations with the corresponding algebraic models. Recall that the $S^{r}$-colored triply-graded homology of the unknot has even generators $u_{1}, \ldots, u_{r}$ and odd generators $\xi_{1}, \ldots, \xi_{r}$ such that

$$
\left[a, q, t_{c}\right] u_{i}=(0,2 i, 2 i-2), \quad\left[a, q, t_{c}\right] \xi_{i}=(2,2 i-2,2 i-1) .
$$

The differential $d_{2 \mid 0}$ is defined by $d_{2 \mid 0}\left(\xi_{i}\right)=\sum_{j=1}^{i} u_{j} u_{i+1-j}$, in particular,

$$
\begin{aligned}
& d_{2 \mid 0}\left(\xi_{1}\right)=u_{1}^{2}, \\
& d_{2 \mid 0}\left(\xi_{2}\right)=2 u_{1} u_{2}, \quad d_{2 \mid 0}\left(\xi_{3}\right)=u_{2}^{2}+2 u_{1} u_{3}, \\
& d_{2 \mid 0}\left(\xi_{4}\right)=2 u_{1} u_{4}+2 u_{2} u_{3} .
\end{aligned}
$$

The uncolored HOMFLYPT homology of the unknot is a free algebra generated by $u_{1}$ and $\xi_{1}$, so the uncolored $\operatorname{sl}(2)$ homology is two-dimensional and spanned by 1 and $u_{1}$.

Using (C.23), one can check that the $\left(\mathrm{sl}(2), S^{2}\right)$ homology of the unknot is spanned by

$$
1, u_{1}, \mu_{1}, u_{2}^{k}, u_{2}^{k} \mu_{1}, k \geq 1,
$$

where $\mu_{1}=2 u_{2} \xi_{1}-u_{1} \xi_{2}$. Note that the homology has nontrivial $\mathbb{Z}_{2}$-torsion, which we do not consider here; see [39] for more details.

The reduced triply-graded homology of the trefoil is three-dimensional and spanned by $1, u_{2}, \xi_{2}$. Therefore the uncolored sl(2) homology of the trefoil can be considered as a quotient of the $\left(\mathrm{sl}(2), S^{2}\right)$ homology of the unknot by $u_{2}^{2}$ and $u_{2} \mu_{1}$, hence it is spanned by $1, u_{1}, u_{2}$ and $\mu_{1}$.

Finally, consider the $\left(\operatorname{sl}(2), S^{2}\right)$ homology of the trefoil. Following (5.15), the reduced $S^{2}$ HOMFLYPT homology of the trefoil is spanned by

$$
1, u_{3}, u_{4}, u_{3}^{2}, \xi_{3}, \xi_{4}, u_{3} \xi_{3}, u_{4} \xi_{3}=-u_{3} \xi_{4}, \xi_{3} \xi_{4} .
$$

For simplicity, let us focus on the bottom row. By our assumption, the unreduced $S^{2}$ HOMFLYPT homology of the trefoil is the tensor product of the unreduced $S^{2}$ HOMFLYPT homology of the unknot and the reduced $S^{2}$ HOMFLYPT homology of the trefoil. The cokernel of $d_{2 \mid 0}$ on the bottom row of the unknot part is spanned by $1, u_{1}$ and $u_{2}^{k}$ for $k \geq 1$, while $d_{2 \mid 0}$ is trivial on the reduced homology of the unknot. Therefore, the bottom row of $\left(\mathrm{sl}(2), S^{2}\right)$ HOMFLYPT homology is contained in

$$
\left\langle 1, u_{1}, u_{2}^{k}\right\rangle \otimes\left\langle 1, u_{3}, u_{4}, u_{3}^{2}\right\rangle .
$$

Using (C.23), we can eliminate $u_{1} u_{3}$ and $u_{1} u_{4}$. Moreover,

$$
\begin{aligned}
u_{1} u_{3}^{2} & =d_{2 \mid 0}\left(\frac{1}{2} u_{3} \xi_{3}-\frac{1}{4} u_{2} \xi_{4}+\frac{1}{4} u_{4} \xi_{2}\right), & u_{2}^{2} u_{3} & =d_{2 \mid 0}\left(\frac{1}{2} u_{2} \xi_{4}-\frac{1}{2} u_{4} \xi_{2}\right), \\
u_{2}^{2} u_{4} & =d_{2 \mid 0}\left(\xi_{3} u_{4}-\xi_{4} u_{3}\right)+2 u_{2} u_{3}^{2}, & u_{2}^{3} & =d_{2 \mid 0}\left(u_{2} \xi_{3}-u_{3} \xi_{2}\right),
\end{aligned}
$$


and these can be eliminated too. Therefore the bottom row of the unreduced $\left(\operatorname{sl}(2), S^{2}\right)$ homology of the trefoil is spanned by

$$
1, u_{1}, u_{2}, u_{3}, u_{4}, u_{2}^{2}, u_{2} u_{4}, u_{2} u_{3}, u_{2}^{k} u_{3}^{2}, k \geq 0 .
$$

The homology of $d_{2 \mid 0}$ on higher levels can be computed by similar methods.

Acknowledgements. We are grateful to M. Abouzaid, M. Aganagic, J. M. Baptista, M. Bershtein, I. Cherednik, K. Costello, R. Elliot, P. Etingof, A. Gorsky, K. Hikami, M. Khovanov, B. Kim, A. N. Kirillov and A. A. Kirillov Jr., I. Losev, A. Morozov, H. Nakajima, A. Negut, N. Nekrasov, A. Oblomkov, A. Okounkov, J. Rasmussen, L. Rozansky, S. Shakirov, V. Shende, C. Vafa, O. Viro, E. Witten, and C. Woodward for useful discussions.

E.G. would like to thank California Institute of Technology and Kyoto Research Institute for Mathematical Sciences for hospitality. The research of E.G. is partially supported by the NSF grant DMS-1559338, grants RFBR10-01-678, NSh-8462.2010.1, Simons Foundation and Russian Academic Excellence Project 5-100.

S.G. would like to thank Instituto Superior Técnico in Lisbon and the Simons Center for Geometry and Physics at Stony Brook for hospitality during the key stages of this work. The work of S.G. is supported in part by DOE grant DE-FG03-92-ER40701FG-02 and in part by NSF grant PHY0757647 .

M.S. would like to thank the California Institute of Technology for hospitality while part of this work was done. The work of S.G. and M.S. was partially supported by ERC Starting Grant no. 335739 "Quantum fields and knot homologies" funded by the European Research Council under the European Union Seventh Framework Programme. M.S. was also partially supported by the Portuguese Fundação para a Ciência e a Tecnologia through the project PTDC/MAT/101503/2008, New Geometry and Topology, and by the Ministry of Science of Serbia, project no. 174012. Opinions and conclusions expressed here are those of the authors and do not necessarily reflect the views of the funding agencies.

\section{References}

[1] M. Abouzaid, On the Fukaya categories of higher genus surfaces, Adv. Math. 217 (2008), 1192-1235.

[2] M. Aganagic and S. Shakirov, Knot homology from refined Chern-Simons theory, arXiv:1105.5117v2 (2012).

[3] M. Aganagic and C. Vafa, Mirror symmetry and supermanifold, Adv. Theor. Math. Phys. 8 (2004), 939-954.

[4] R. Auzzi, M. Shifman and A. Yung, Composite non-Abelian flux tubes in $N=2$ SQCD, Phys. Rev. D 73 (2006), 105012; Erratum, ibid. 76 (2007), 109901. 
[5] J. M. Baptista, Non-abelian vortices, Hecke modifications and singular monopoles, Lett. Math. Phys. 92 (2010), 243-252.

[6] A. Berele and A. Regev, Hook Young diagrams with applications to combinatorics and to representations of Lie superalgebras, Adv. Math. 64 (1987), 118-175.

[7] A. Berele and A. Regev, Hook Young diagrams, combinatorics and representations of Lie superalgebras, Bull. Amer. Math. Soc. (N.S.) 8 (1983), 337-339.

[8] S. B. Bradlow and O. García-Prada, Stable triples, equivariant bundles and dimensional reduction, Math. Ann. 304 (1996), 225-252.

[9] S. B. Bradlow and O. García-Prada, Non-abelian monopoles and vortices, in: Geometry and Physics (Aarhus, 1995), Lecture Notes in Pure Appl. Math. 184, Dekker, New York, 1997, 567-589.

[10] L. Carini and J. B. Remmel, Formulas for the expansion of the plethysms $s_{2}\left[s_{(a, b)}\right]$ and $s_{2}\left[s_{\left(n^{k}\right)}\right]$, Discrete Math. 193 (1998), 147-177.

[11] S. Cautis, Clasp technology to knot homology via the affine Grassmannian, Math. Ann. 363 (2015), 1053-1115.

[12] S. Cautis and J. Kamnitzer, Knot homology via derived categories of coherent sheaves II, $\mathfrak{s l}_{m}$ case, Invent. Math. 174 (2008), 165-232.

[13] S. Cautis and J. Kamnitzer, Knot homology via derived categories of coherent sheaves IV, coloured links, Quantum Topol. 8 (2017), 381-411.

[14] I. Cherednik, Jones polynomials of torus knots via DAHA, Int. Math. Res. Notices 2013, 5366-5425.

[15] B. Cooper, M. Hogancamp and V. Krushkal, $\mathrm{SO}(3)$ homology of graphs and links, Algebr. Geom. Topol. 11 (2011), 2137-2166.

[16] B. Cooper and V. Krushkal, Categorification of the Jones-Wenzl projectors, Quantum Topol. 3 (2012), 139-180.

[17] K. Costello, Topological conformal field theories and Calabi-Yau categories, Adv. Math. 210 (2007), 165-214.

[18] T. Dimofte and S. Gukov, Refined, motivic, and quantum, Lett. Math. Phys. 91 (2010), 1-27.

[19] T. Dimofte, S. Gukov and L. Hollands, Vortex counting and Lagrangian 3-manifolds, Lett. Math. Phys. 98 (2011), 225-287.

[20] N. M. Dunfield, S. Gukov and J. Rasmussen, The superpolynomial for knot homologies, Experiment. Math. 15 (2006), 129-159.

[21] P. Dunin-Barkowski, A. Mironov, A. Morozov, A. Sleptsov and A. Smirnov, Superpolynomials for torus knots from evolution induced by cut-and-join operators, J. High Energy Phys. 2013, no. 3, 021, 85 pp.

[22] B. Elias and M. Hogancamp, On the computation of torus link homology, arXiv: 1603.00407 (2016).

[23] P. Etingof, E. Gorsky and I. Losev, Representations of rational Cherednik algebras with minimal support and torus knots, Adv. Math. 277 (2015), 124-180.

[24] P. Etingof and X. Ma, Lecture notes on Cherednik algebras, arXiv:1001.0432 (2010).

[25] M. Eto, Y. Isozumi, M. Nitta, K. Ohashi and N. Sakai, Moduli space of non-Abelian vortices, Phys. Rev. Lett. 96 (2006), no. 16, 161601.

[26] M. Eto, K. Konishi, G. Marmorini, M. Nitta, K. Ohashi, W. Vinci and N. Yokoi, Non-Abelian vortices of higher winding numbers, Phys. Rev. D 74 (2006), no. 6, 065021.

[27] E. Frenkel and E. Witten, Geometric endoscopy and mirror symmetry, Comm. Number Theory Phys. 2 (2008), 113-283.

[28] I. Frenkel, C. Stroppel and J. Sussan, Categorifying fractional Euler characteristics, Jones-Wenzl projectors and 3j-symbols, Quantum Topol. 3 (2012), 181-253. 
[29] H. Fuji, S. Gukov, M. Stošić and P. Sułkowski, 3d analogs of Argyres-Douglas theories and knot homologies, J. High Energy Phys. 2013, no. 1, 175, 37 pp.

[30] H. Fuji, S. Gukov, P. Sułkowski and H. Awata, Volume conjecture: refined and categorified, Adv. Theor. Math. Phys. 16 (2012), 1669-1777.

[31] H. Fuji, S. Gukov and P. Sułkowski, Super-A-polynomial for knots and BPS states, Nuclear Phys. B 867 (2013), 506-546.

[32] R. S. Garavuso, L. Katzarkov, M. Kreuzer and A. Noll, Super Landau-Ginzburg mirrors and algebraic cycles, J. High Energy Phys. 2011, no. 3, 017, 28 pp.: Erratum ibid. 2011, no. 8, 063, 5 pp.

[33] M. Gerstenhaber and S. D. Schack, Algebras, bialgebras, quantum groups, and algebraic deformations, in: Deformation Theory and Quantum Groups with Applications to Mathematical Physics, Contemp. Math. 134, Amer. Math. Soc., Providence, RI, 1992, 51-92; A Hodge-type decomposition for commutative algebra cohomology, J. Pure Appl. Algebra 48 (1987), 229-247.

[34] R. Gopakumar and C. Vafa, On the gauge theory/geometry correspondence, Adv. Theor. Math. Phys. 3 (1999), 1415-1443.

[35] E. Gorsky, q,t-Catalan numbers and knot homology, in: Zeta Functions in Algebra and Geometry, Contemp. Math. 566, Amer. Math. Soc., Providence, RI, 2012, 212 232.

[36] E. Gorsky, Arc spaces and DAHA representations, Selecta Math. (N.S.) 19 (2013), $125-140$.

[37] E. Gorsky and A. Negut, Refined knot invariants and Hilbert schemes, J. Math. Pures Appl. (9) 104 (2015), 403-435.

[38] E. Gorsky, A. Negut and J. Rasmussen, Flag Hilbert schemes, colored projectors and Khovanov-Rozansky homology, arXiv:1608.07308 (2016).

[39] E. Gorsky, A. Oblomkov and J. Rasmussen, On stable Khovanov homology of torus knots, Experiment. Math. 22 (2013), 265-281.

[40] E. Gorsky, A. Oblomkov, J. Rasmussen and V. Shende, Torus knots and the rational DAHA, Duke Math. J. 163 (2014), 2709-2794.

[41] S. Gukov, Gauge theory and knot homologies, Fortschr. Phys. 55 (2007), 473-490.

[42] S. Gukov and I. Saberi, Lectures on knot homology and quantum curves, arXiv:1211. 6075 (2012).

[43] S. Gukov, A. S. Schwarz and C. Vafa, Khovanov-Rozansky homology and topological strings, Lett. Math. Phys. 74 (2005), 53-74.

[44] S. Gukov and M. Stošić, Homological algebra of knots and BPS states, in: StringMath 2011, Proc. Sympos. Pure Math. 85, Amer. Math. Soc., Providence, RI, 2012, $125-171$.

[45] S. Gukov and J. Walcher, Matrix factorizations and Kauffman homology, arXiv: hep-th/0512298 (2005).

[46] S. Gukov and E. Witten, Gauge theory, ramification, and the geometric Langlands program, in: Current Developments in Mathematics, 2006, Int. Press, Somerville, MA, 2008, 35-180.

[47] S. M. Gusein-Zade and A. Varchenko, Verlinde algebras and the intersection form on vanishing cycles, Selecta Math. (N.S.) 3 (1997), 79-97.

[48] K. Hashimoto and D. Tong, Reconnection of non-Abelian cosmic strings, J. Cosmol. Astropart. Phys. 2005, no. 2, 004, 20 pp.

[49] G. Hochschild, B. Kostant and A. Rosenberg, Differential forms on regular affine algebras, Trans. Amer. Math. Soc. 102 (1962), 383-408.

[50] M. Hogancamp, Stable homology of torus links via categorified Young symmetrizers I: one-row partitions, arXiv:1505.08148v1 (2015). 
[51] M. Ionel, S. Karigiannis and M. Min-Oo, Bundle constructions of calibrated submanifolds in $\mathbb{R}^{7}$ and $\mathbb{R}^{8}$, Math. Res. Lett. 12 (2005), 493-512.

[52] A. Iqbal and C. Kozçaz, Refined Hopf link revisited, J. High Energy Phys. 2012, no. 4, 046, 18 pp.

[53] D. L. Jafferis and G. W. Moore, Wall crossing in local Calabi-Yau manifolds, arXiv:0810.4909 (2008).

[54] S. Karigiannis and M. Min-Oo, Calibrated subbundles in non-compact manifolds of special holonomy, Ann. Global Anal. Geom. 28 (2005), 371-394.

[55] S. Katz, A. Klemm and C. Vafa, Geometric engineering of quantum field theories, Nuclear Phys. B 497 (1997), 173-195.

[56] M. Khovanov, Categorification of the Jones polynomial, Duke Math. J. 101 (2000), $359-426$.

[57] M. Khovanov and L. Rozansky, Matrix factorizations and link homology, Fund. Math. 199 (2008), 1-91.

[58] M. Khovanov and L. Rozansky, Matrix factorizations and link homology II, Geom. Topol. 12 (2008), 1387-1425.

[59] A. A. Kirillov and I. M. Pak, Covariants of the symmetric group and its analogues in Weyl algebras, Funct. Anal. Appl. 24 (1990), 172-176.

[60] M. Kontsevich, Homological algebra of mirror symmetry, in: Proc. Int. Congress of Mathematicians (Zürich, 1994), Birkhäuser, Basel, 1995, Vol. 1, 120-139.

[61] M. Kontsevich and Y. Soibelman, Notes on $A_{\infty}$-algebras, $A_{\infty}$-categories and noncommutative geometry, in: Homological Mirror Symmetry, Lecture Notes in Phys. 757, Springer, Berlin, 2009, 153-219.

[62] S. Koshkin, Conormal bundles to knots and the Gopakumar-Vafa conjecture, Adv. Theor. Math. Phys. 11 (2007), 591-634.

[63] J. M. F. Labastida, M. Mariño and C. Vafa, Knots, links and branes at large N, J. High Energy Phys. 2000, no. 11, 007, 42 pp.

[64] X.-S. Lin and H. Zheng, On the Hecke algebras and the colored HOMFLYPT polynomial, Trans. Amer. Math. Soc. 362 (2010), 1-18.

[65] I. G. Macdonald, A new class of symmetric functions, Actes du 20e Séminaire Lotharingien de Combinatoire B20a, Publ. I.R.M.A. Strasbourg 372/S-20 (1988), 131-171.

[66] V. Mikhaylov and E. Witten, Branes and supergroups, Comm. Math. Phys. 340 (2015), 699-832.

[67] V. Molchanov, On the Poincaré series of representations of finite reflection groups, Funct. Anal. Appl. 26 (1992), 143-145.

[68] K. Nagao and H. Nakajima, Counting invariant of perverse coherent sheaves and its wall-crossing, Int. Math. Res. Notices 2011, 3885-3938.

[69] H. Nakajima, Lectures on Hilbert Schemes of Points on Surfaces, Amer. Math. Soc., Providence, RI, 1999.

[70] H. Nakajima and K. Yoshioka, Lectures on instanton counting, in: Algebraic Structures and Moduli Spaces, CRM Proc. Lecture Notes 38, Amer. Math. Soc., Providence, RI, 2004, 31-101.

[71] S. Nawata, P. Ramadevi, Zodinmawia and X. Sun, Super-A-polynomials for twist knots, J. High Energy Phys. 2012, no. 11, 157, 38 pp.

[72] N. Nekrasov and S. Shadchin, ABCD of instantons, Comm. Math. Phys. 252 (2004), 359-391.

[73] H. Ooguri and C. Vafa, Knot invariants and topological strings, Nuclear Phys. B 577 (2000), 419-438.

[74] D. O. Orlov, Triangulated categories of singularities and D-branes in Landau-Ginzburg models, Proc. Steklov Inst. Math. 2004, no. 3 (246), 227-248. 
[75] H. Queffelec and A. Sartori, Mixed quantum skew Howe duality and link invariants of type A, arXiv:1504.01225 (2015).

[76] H. Queffelec and A. Sartori, A note on the $\mathfrak{g l}_{m \mid n}$ link invariants and the HOMFLYPT polynomial, arXiv:1506.03329 (2015).

[77] J. Rasmussen, Khovanov homology and the slice genus, Invent. Math. 182 (2010), 419-447.

[78] J. Rasmussen, Some differentials on Khovanov-Rozansky homology, Geom. Topol. 19 (2015), 3031-3104.

[79] M. Rosso and V. Jones, On the invariants of torus knots derived from quantum groups, J. Knot Theory Ramif. 2 (1993), 97-112.

[80] L. Rozansky, An infinite torus braid yields a categorified Jones-Wenzl projector, Fund. Math. 225 (2014), 305-326.

[81] A. Sartori, The Alexander polynomial as quantum invariant of links, Ark. Mat. 53 (2015), 177-202.

[82] A. Schwarz, Sigma-models having supermanifolds as target spaces, Lett. Math. Phys. 38 (1996), 91-96.

[83] E. Sharpe, Lectures on D-branes and sheaves, arXiv:hep-th/0307245 (2003).

[84] S. Sethi, Supermanifolds, rigid manifolds and mirror symmetry, Nuclear Phys. B 430 (1994), 31-50.

[85] S. Stevan, Chern-Simons invariants of torus links, Ann. Henri Poincaré 11 (2010), 1201-1224.

[86] M. Stošić, Homological thickness and stability of torus knots, Algebr. Geom. Topol. 7 (2007), 261-284.

[87] A. Strominger, S.-T. Yau and E. Zaslow, Mirror symmetry is T-duality, Nuclear Phys. B 479 (1996), 243-259.

[88] K. Taipale, K-theoretic J-functions of type A flag varieties, Int. Math. Res. Notices 2013, 3647-3677.

[89] C. Taubes, Lagrangians for the Gopakumar-Vafa conjecture, in: The Interaction of Finite-Type and Bromov-Witten Invariants (BIRS 2003), Geom. Topol. Monogr. 8, Geom. Topol. Publ., Coventry, 2006, 73-95.

[90] D. Tubbenhauer, P. Vaz and P. Wedrich, Super q-Howe duality and web categories, Algebr. Geom. Topol. 17 (2017), 3703-3749.

[91] C. Vafa, Brane/anti-brane systems and $U(N \mid M)$ supergroup, arXiv:hep-th/0101218 (2001).

[92] B. Webster, Knot invariants and higher representation theory II: the categorification of quantum knot invariants, arXiv:1005.4559v7 (2013).

[93] P. Wedrich, Exponential growth of colored HOMFLY-PT homology, arXiv:1602. 02769 (2016).

[94] E. Witten, Quantum field theory and Jones polynomial, Comm. Math. Phys. 121 (1989), 351-399.

[95] E. Witten, D-branes and K-theory, J. High Energy Phys. 1998, no. 12, 019, 41 pp.

[96] E. Witten, Fivebranes and knots, Quantum Topol. 3 (2012), 1-137.

[97] E. Witten, Perturbative gauge theory as a string theory in twistor space, Comm. Math. Phys. 252 (2004), 189-258.

[98] H. Wu, A colored $\mathfrak{s l}(N)$ homology for links in $S^{3}$, Dissertationes Math. 499 (2014).

[99] A. Yekutieli, The continuous Hochschild cochain complex of a scheme, Canad. J. Math. 54 (2002), 1319-1337.

[100] Y. Yonezawa, Quantum $\left(\mathfrak{s l}_{n}, \wedge V_{n}\right)$ link invariant and matrix factorizations, Nagoya Math. J. 204 (2011), 69-123. 
Eugene Gorsky

Department of Mathematics

UC Davis

One Shields Avenue

Davis, CA 95616, U.S.A.

and

National Research University

Higher School of Economics

Moscow, Russia

E-mail: egorskiy@math.ucdavis.edu

Marko Stošić

CAMGSD

Departamento de Matemática

Instituto Superior Técnico

Av. Rovisco Pais

1049-001 Lisbon, Portugal

and

Mathematical Institute SANU

Knez Mihailova 36

11000 Belgrade, Serbia

E-mail: mstosic@isr.ist.utl.pt
Sergei Gukov

California Institute of Technology Pasadena, CA 91125, U.S.A. and Max-Planck-Institut für Mathematik Vivatsgasse 7 D-53111 Bonn, Germany E-mail: gukov@theory.caltech.edu 
